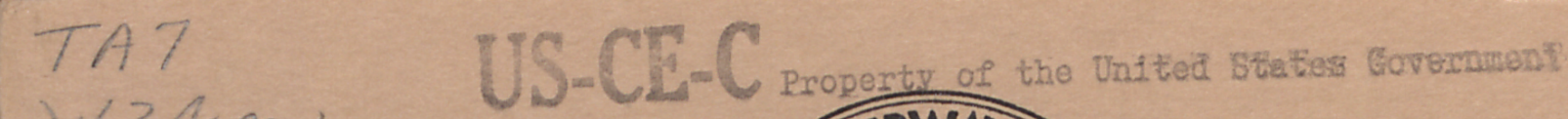

W $34 \mathrm{~m}$

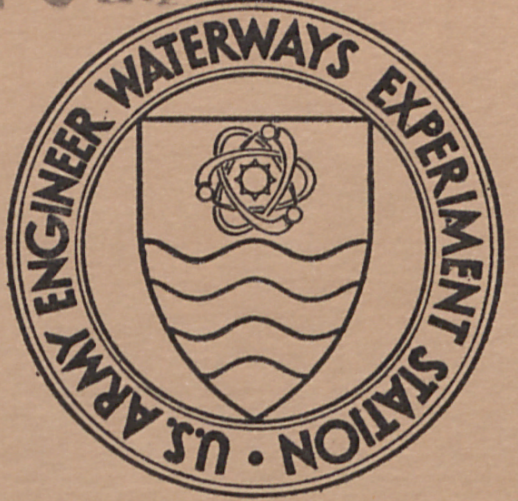

MISCELLANEOUS PAPER S-74-17

\title{
HELICOPTER DOWNWASH DATA
}

by

G. W. Leese, J. T. Knight, Jr.
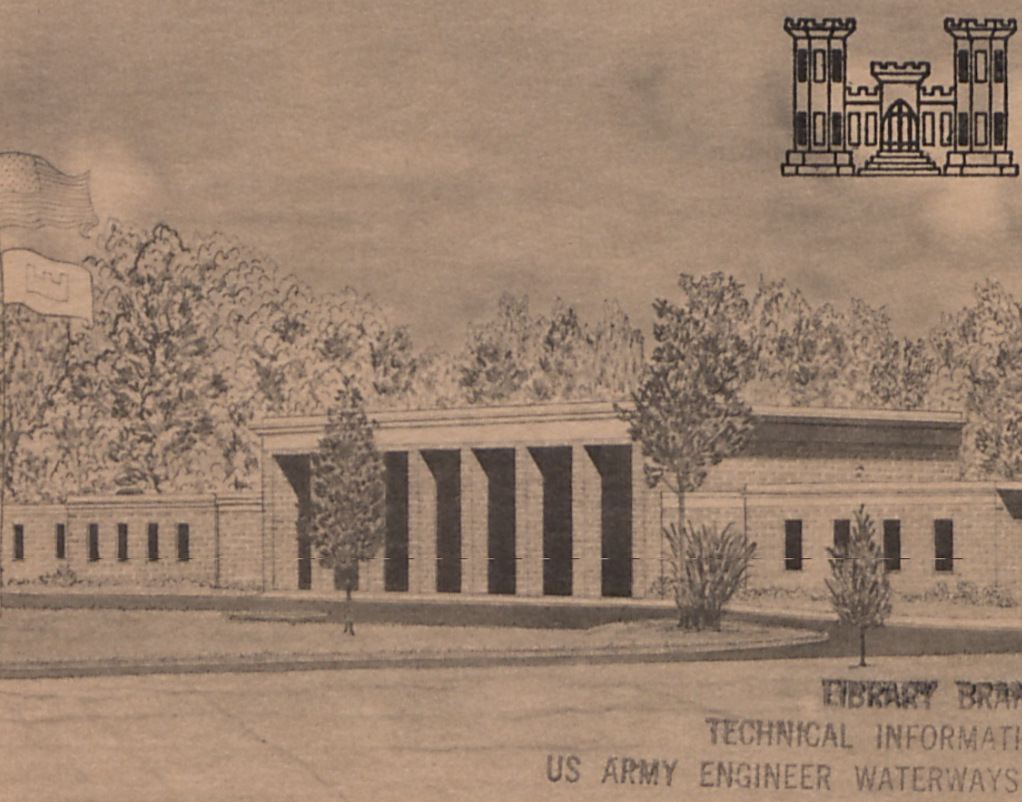

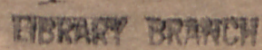

RECHNHCAL INFORMATION CONTES

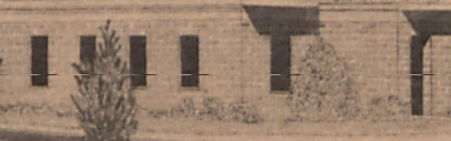

1.0.

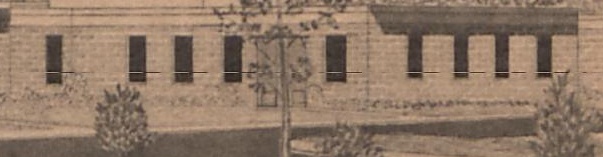

ENGINEER WATERWAYS EXPERIMEIYI STATION

VICKSBURE. MISSISSIPPI

June 1974

Sponsored by U. S. Army Materiel Command

Conducted by U. S. Army Engineer Waterways Experiment Station

Soils and Pavements Laboratory

Vicksburg, Mississippi 


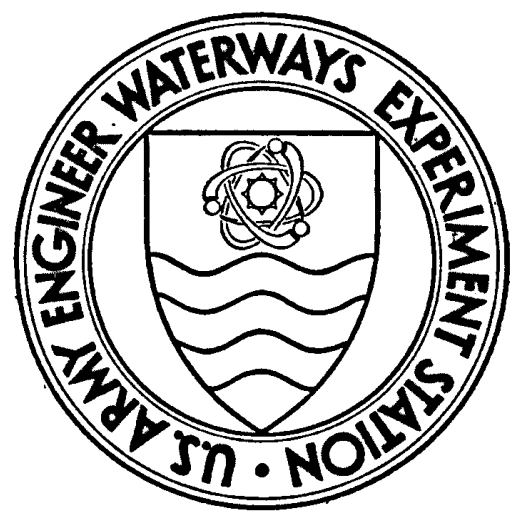

MISCELLANEOUS PAPER S-74-17

\section{HELICOPTER DOWNWASH DATA \\ by}

G. W. Leese, J. T. Knight, Jr.

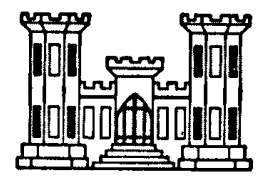

June 1974

Sponsored by U. S. Army Materiel Command.

Project No. IT062013A046, Task 05

Conducted by U. S. Army Engineer Waterways Experiment Station

Soils and Pavements Laboratory

Vicksburg, Mississippi 
FOREWORD

The general authorization for this investigation is contained in Research and Development Project 1 T062013A046 (Task 05), "Trafficability and Mobility Research." The investigation was performed under sponsorship of the Research, Development and Engineering Directorate, U. S. Army Materiel Command. Also participating in the investigation by making arrangements for and scheduling of aircraft and operating personnel was the U. S. Army Aeromedical Research Laboratory (ARL), Ft. Rucker, Ala.

The data reported herein were obtained during September and october 1971 by personnel of the U. S. Army Engineer Waterways Experiment Station (WES) Soils and Pavements Laboratory under the general supervision of Messrs. J. P. Sale and W. L. McInnis, and under the direct supervision of Mr. G. W. Ieese. Personnel of the ARI assisting WES were under the command of COL R. W. Bailey and under the direct supervision of COL W. P. Shane and CPT G. R. McCahan, Jr. This report was prepared by Messrs. G. W. Leese and J. T. Knight, Jr.

Special acknowledgment is made to the U. S. Army Aviation Test Board of Ft. Rucker, Ala., for supplying the aircraft and operating personnel needed during this investigation.

Directors of WES during the conduct of this investigation and the preparation and publication of this report were BG E. D. Peixotto, CE, and COL G. H. Hilt, CE. Technical Director was Mr. F. R. Brown. 


\section{CONTENTS}

$\underline{\text { Page }}$

FOREWORD . . . . . . . . . . . . . . . . . . . . . iii

CONVERSION FACTORS, BRITISH TO METRIC UNITS OF MEASUREMENT • • . vii SUMMARY • . . . . . . . . . . . . . . . . . . . . . . ix

PART I: INTRODUCTION . . . . . . . . . . . . . . . . . 1

Background . . . . . . . . . . . . . . . . . 1

Purpose .. . . . . . . . . . . . . . . . . 1

Scope ............................ . . . 1

PART II: INSTRUMENTATION AND AIRCRAFT . . . . . . . . . . . . 3

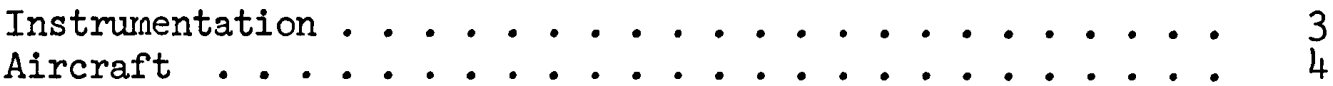

PART III: TESTS AND DATA . . . . . . . . . . . . . . . 9

Test Procedure ..................... . . . . 9

Data .. . . . . . . . . . . . . . . . . 9

TABLES $1-19$ 
CONVERSION FACTORS, BRITISH TO METRIC UNITS OF MEASUREMENT

British units of measurement used in this report can be converted to metric units as follows:

Multiply

inches

feet

square feet

pounds (mass)

pounds (force)

pounds (mass) per square foot

pounds (force) per square foot

miles per hour (U. S. statute)

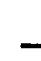

By

$$
2.54
$$

0.3048

0.092903

0.4535924

4.448222

4.882428

47.88026

1.609344
To Obtain

centimeters

meters

square meters

kilograms

newtons

kilograms per square meter newtons per square meter kilometers per hour 


\section{SUMMARY}

This data report describes an investigation involving measurement of horizontal velocities, along and up to $6 \mathrm{ft}$ above the ground surface, generated by the rotor systems of various Army helicopters during takeoff, hover, and fly-by operations.

Measurements of ground-wash velocities during various operational modes were collected for $\mathrm{OH}-58 \mathrm{~A}, \mathrm{OH}-6 \mathrm{~A}, \mathrm{AH}-1 \mathrm{G}, \mathrm{UH}-1 \mathrm{H}, \mathrm{UH}-\mathrm{IM}, \mathrm{CH}-47$, and $\mathrm{CH}-54$ helicopters.

These data are presented for record only with analyses being beyond the scope of this report. 


\section{PART I: INTRODUCTION}

Background

1. The gradual increase in size and load-carrying capacity of the helicopter has created a need for predicting the effect of the rotor downwash on the ground surface, supporting equipment, cargo containers, operating personnel, and expedient surfacing materials. To fully define the ground-wash flow field, velocity measurements are needed under helicopters of various sizes and gross weights. From these flowfield characteristics, design parameters can be established to provide landing and takeoff operational areas for helicopters and to assist in predicting the flow-fiela characteristics of the larger and heavier helicopters of the future.

\section{Purpose}

2. The purpose of this investigation was to measure rotor downwash horizontal velocities along and up to $6 \mathrm{ft}^{*}$ above the ground surface generated by various Army helicopters during takeoff, hover, and fly-by operations.

\section{Scope}

3. Tests were conducted at Ft. Rucker, Ala., with the $\mathrm{CH}-54$ helicopter and at Apalachicola, Fla., utilizing various other Army helicopters operating over an array of instrumentation arranged in such a manner as to obtain ground-wash flow-field velocities produced by the

* A table of factors for converting British units of measurement to metric units is presented on page vii. 
aircraft rotor systems during various modes of operation. Tabulations of the data obtained were made for each velocity measuring station. Analysis of the data was beyond the scope of this investigation. 


\section{Instrumentation}

4. The instrumentation array consisted of a number of windvelocity pickups placed along and above a gage line so as to sense the ground-wash velocities along and up to $6 \mathrm{ft}$ above the ground surface that were generated by the helicopter's rotor system.

\section{Velocity sensors}

5. The wind-velocity sensors were fabricated at the U. S. Army Engineer Waterways Experiment Station (WES) by fitting small induction generators with 4-in.-diam propellers (fig. 1) and exciting the generators with 26-volt, 400-Hz current. The output of the generators was

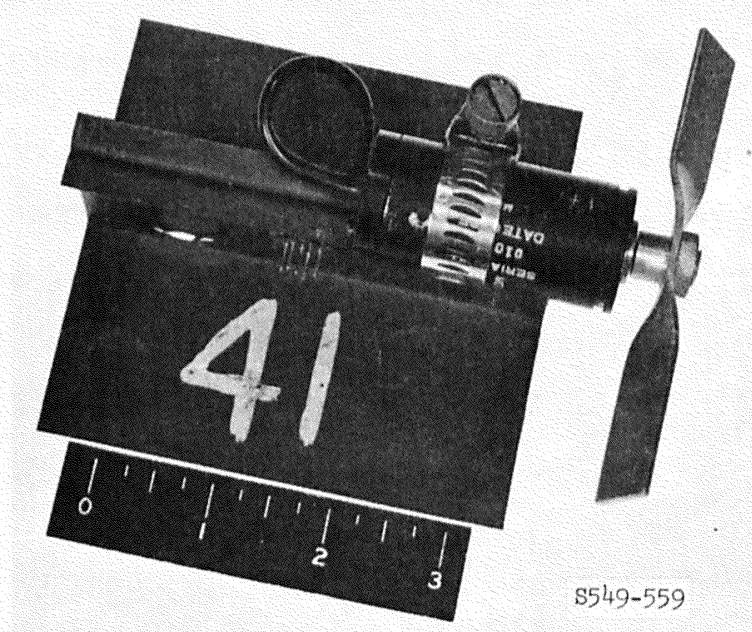

Fig. 1. Velocity sensor

proportional to their rpm's; and by passing this output through a rectifying bridge, it could be recorded using an oscillograph.

6. Tests in the WES Surface Blast Effects Research facility wind tunnel proved the velocity sensors and their electrical circuitry to be accurate and reliable. Calibration curves were established for each sensor before the field tests; wind tunnel tests after the field tests indicated satisfactory performance and accuracy of the sensors. 
7. The test area at Apalachicola consisted of a concrete-surfaced aircraft parking area; the test area at Ft. Rucker was on a section of runway. Test procedures and instrumentation layout were the same at both locations. Velocity sensors were placed on the ground surface along a straight line; horizontal spacing between the sensors was $10 \mathrm{ft}$ as shown in fig. 2 .

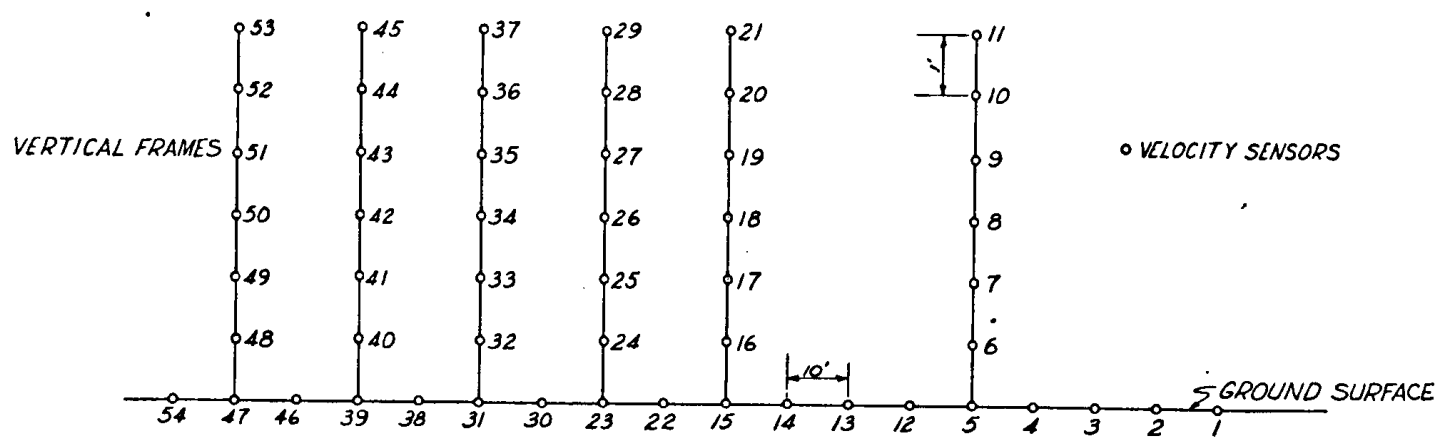

Fig. 2. Layout of velocity sensors

Along this same line, sensors were also mounted on vertical frames to obtain horizontal ground-wash velocities at l-ft vertical intervals up to a height of $6 \mathrm{ft}$ above the ground surface. Fig. 3 shows a close-up of the velocity sensors, and fig. 4 shows a complete vertical array. Fig. 5 is a ground view of the test site at Apalachicola, while fig. 6 is an aerial view of the same test area.

\section{Aircraft}

8. The helicopters utilized in this study were the $0 \mathrm{H}-58 \mathrm{~A}, 0 \mathrm{H}-6 \mathrm{~A}$, $\mathrm{AH}-1 \mathrm{G}, \mathrm{UH}-1 \mathrm{H}, \mathrm{UH}-1 \mathrm{M}, \mathrm{CH}-47$, and $\mathrm{CH}-54$. Pertinent aircraft data are given in the following tabulation:

\begin{tabular}{|c|c|c|c|c|c|c|}
\hline \multirow{2}{*}{$\begin{array}{l}\text { Heli- } \\
\text { copter } \\
\text { Type }\end{array}$} & \multicolumn{2}{|c|}{ Rotor } & \multicolumn{2}{|c|}{ Disc } & \multicolumn{2}{|c|}{ Weight, Ib } \\
\hline & No. & $\begin{array}{l}\text { Diam } \\
\text { ft }\end{array}$ & $\begin{array}{l}\text { Areq } \\
\text { sq ft }\end{array}$ & $\begin{array}{l}\text { Maximum } \\
\text { Load, psf }\end{array}$ & Basic & $\begin{array}{l}\text { Maximum } \\
\text { Tested }\end{array}$ \\
\hline $\mathrm{OH}-58 \mathrm{~A}$ & 1 & 35.33 & 980 & 3.26 & 1,740 & 3,200 \\
\hline $0 \mathrm{H}-6 \mathrm{~A}$ & 1 & 26.3 & 543 & 3.31 & 1,163 & 1,800 \\
\hline $\mathrm{AH}-\mathrm{IG}$ & 1 & 44 & 1520 & 6.25 & 5,676 & 9,500 \\
\hline $\mathrm{UH}-1 \mathrm{H}$ & 1 & 48.1 & 1817 & 5.22 & 4,717 & 9,500 \\
\hline $\mathrm{UH}-\mathrm{IM}$ & 1 & 44.3 & 1541 & 6.16 & 5,080 & 9,500 \\
\hline $\mathrm{CH}-47$ & 2 & 59.1 & 5486 & 6.56 & 18,023 & 36,000 \\
\hline $\mathrm{CH}-54$ & 1 & 72 & 4071 & 11.54 & 19,825 & 47,000 \\
\hline
\end{tabular}




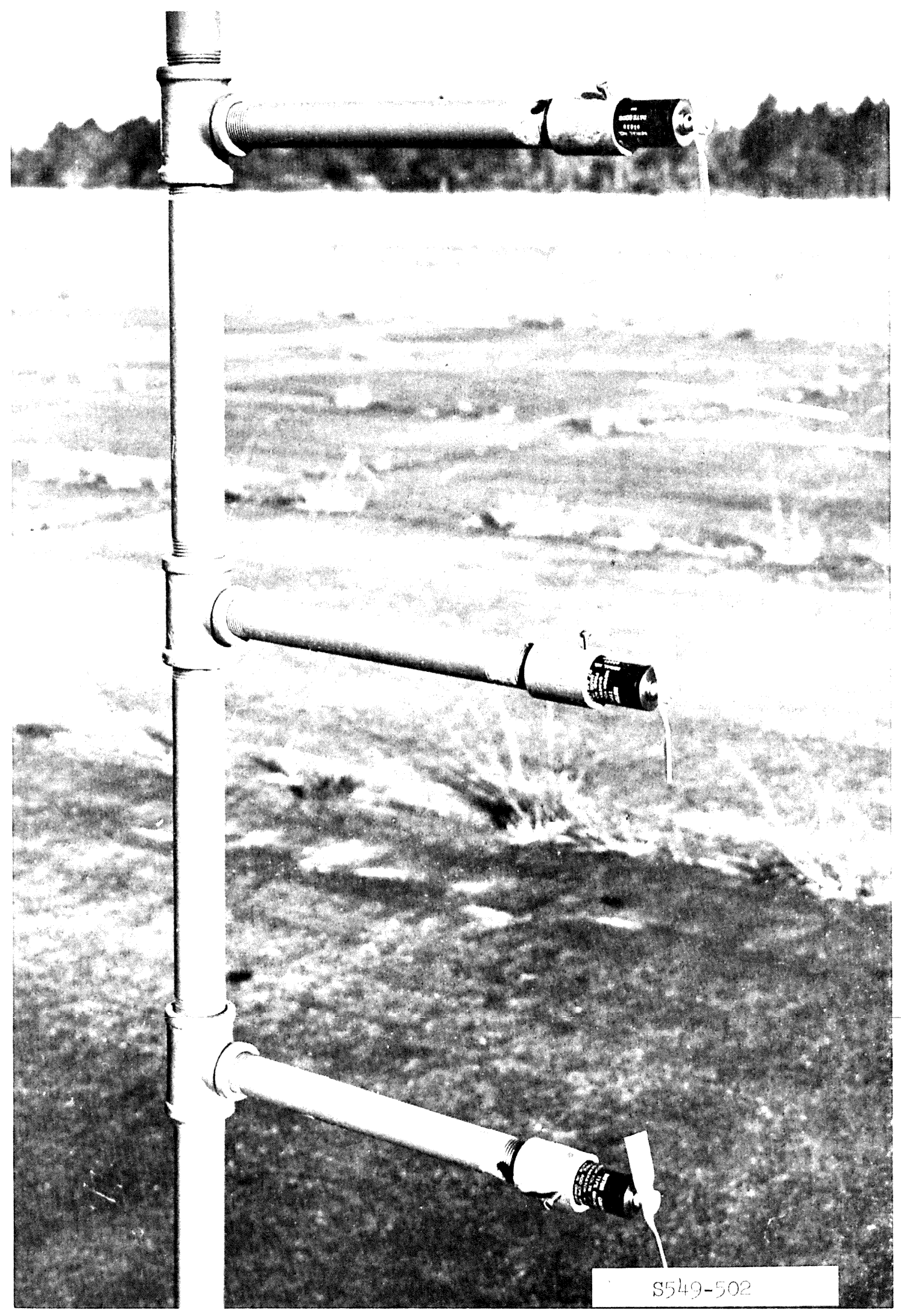

Fig. 3 Close-up of velocity sensors 


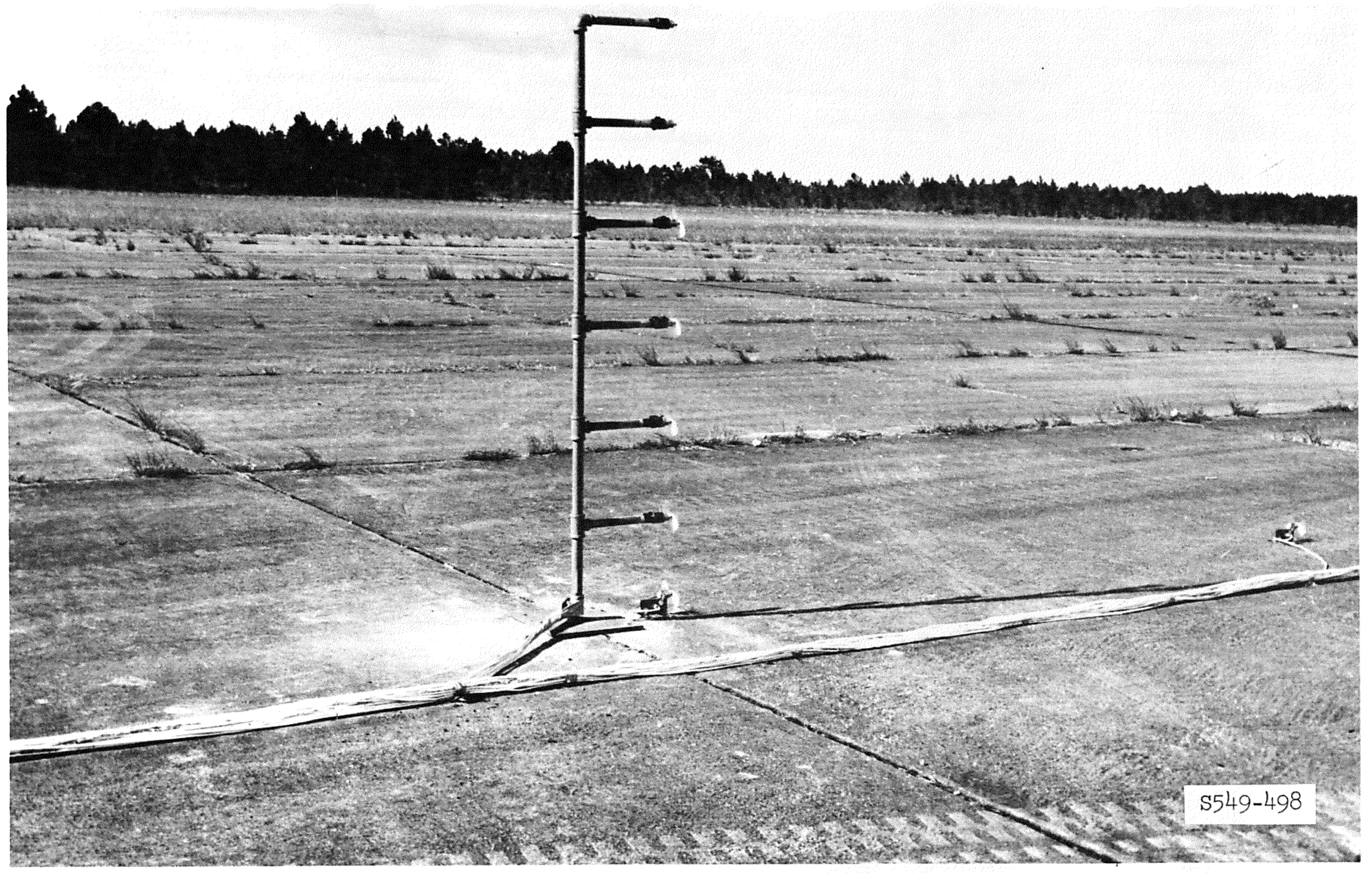

Fig. 4 Single vertical array of velocity sensors 


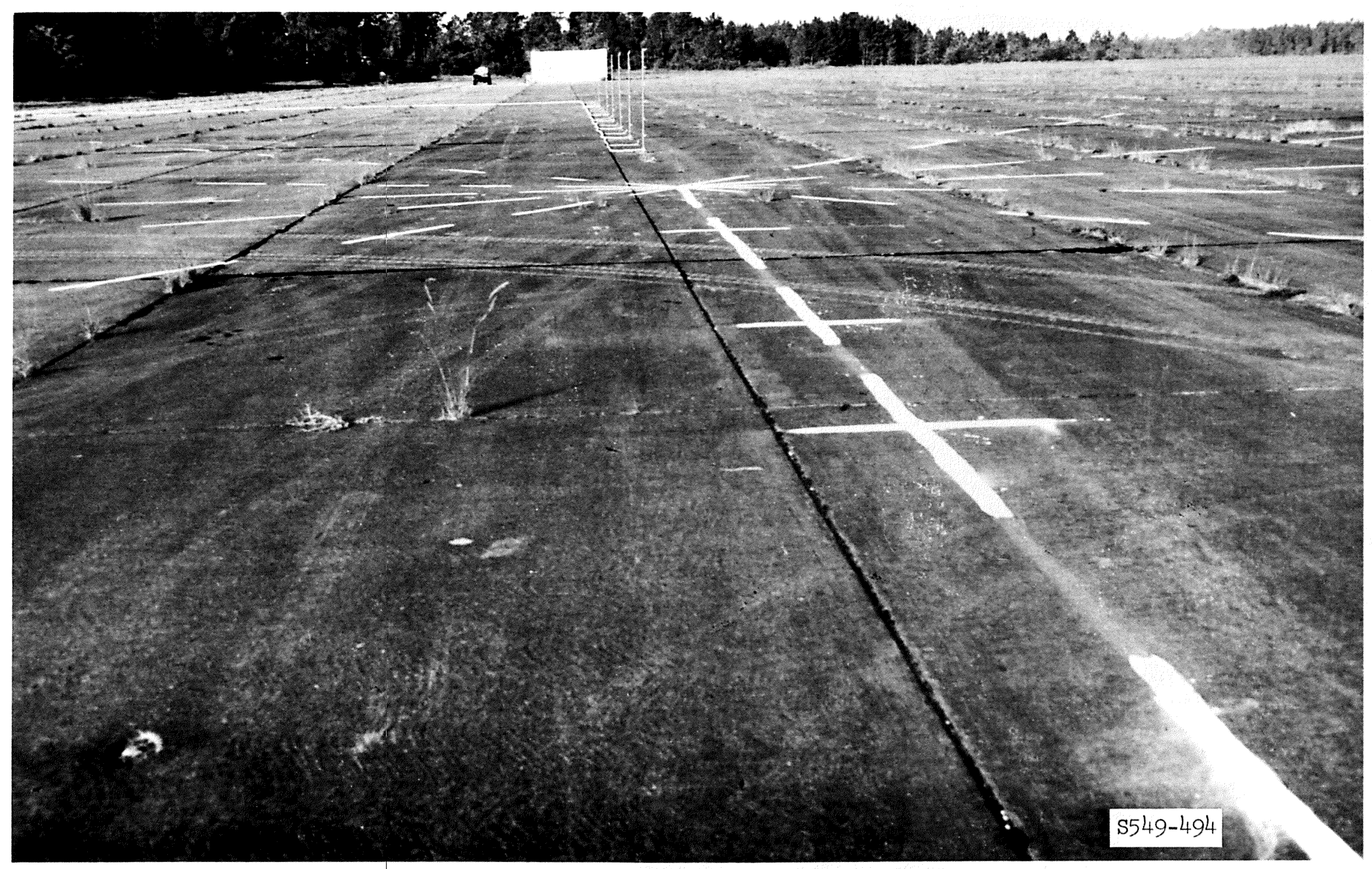

Fig. 5 Ground view of test site at Apalachicola 


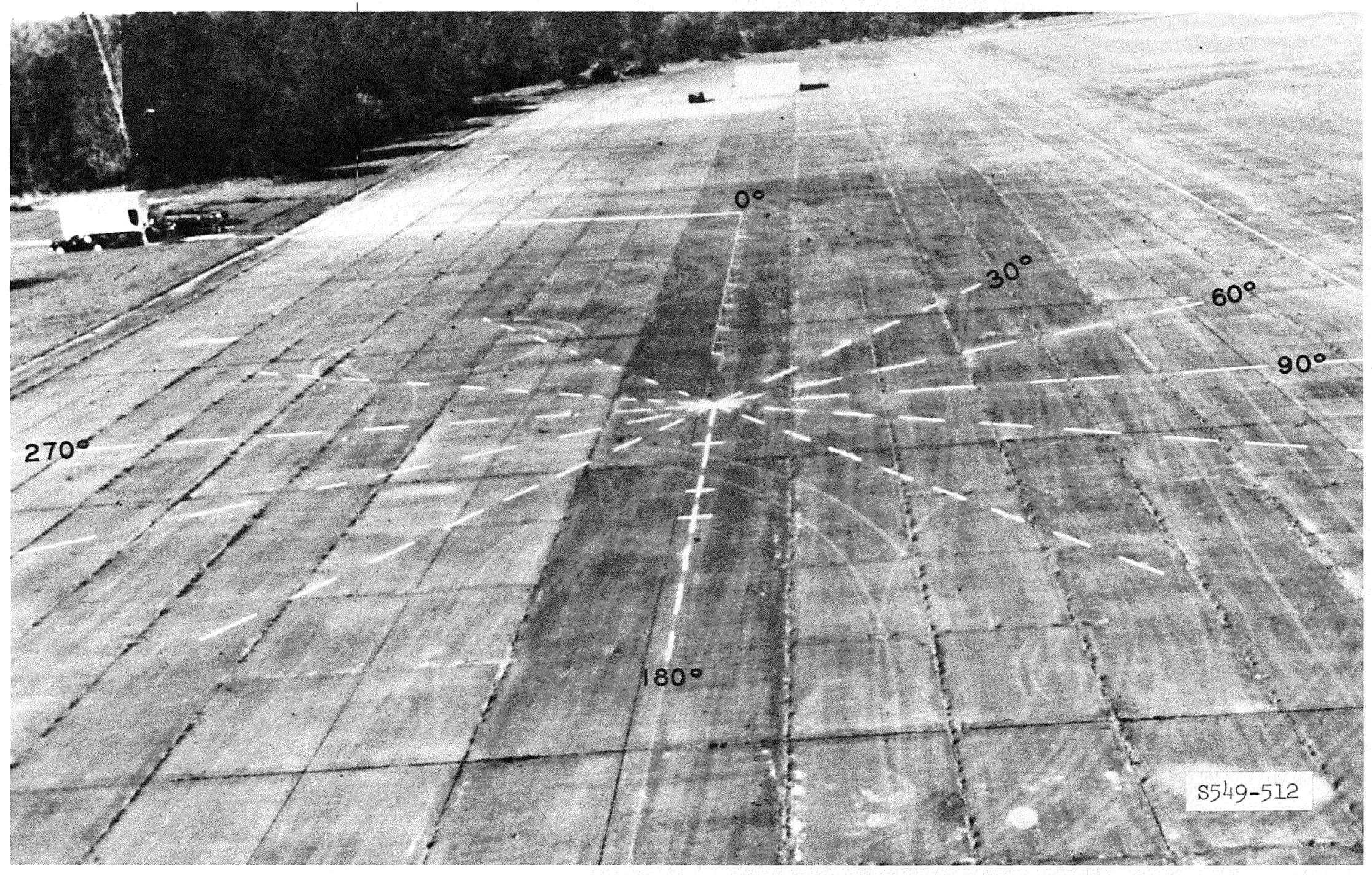

Fig. 6 Aerial view of Apalachicola test site 


\section{Test Procedure}

9. The test plan was to measure the ground-wash horizontal velocities produced by as many different aircraft as could be made available. In order to obtain complete velocity ground-wash flow patterns, the sensors were monitored as the aircraft maintained a constant elevation above a point along the sensor line while the aircraft rotated about the rotor hub. Data were recorded for 30-deg increments of aircraft rotation where possible. Aircraft heading is referenced to the gage line with 0 deg being where the nose of the aircraft was pointing down the gage line; rotation was to the pilot's right (clockwise). The heights of the aircraft are referenced to the rotor actuator plane, hereafter referred to as the rotor.

\section{Data}

OH-58A helicopter

10. Iift-off tests. Tests were made with the OH-58A during simulated lift-offs. To accomplish this the aircraft was flown with the landing gear just clear of the ground so as to ensure total weight on the rotor. This procedure was used to reduce both aircraft flight time and pilot fatigue. Velocity data were recorded at each 30-deg rotation of the aircraft. These data are shown in table 1.

11. Hover tests. Tests were also run with the $0 \mathrm{H}-58 \mathrm{~A}$ hovering over a point on the gage line at a height of $35 \mathrm{ft}$ above the ground surface. Data recordings were made as the aircraft rotated in 90-deg increments. These data are shown in table 2. The aircraft was also hovered over the given point at a height of $13 \mathrm{ft}$ above the surface with data recordings being made at each $30 \mathrm{deg}$ of aircraft rotation. Data for these tests are also shown in table 2 .

12. Fly-by tests. Tests were also made with the $0 H-58 \mathrm{~A}$ flying over a given point and perpendicular to the gage line with two passes 
being made at heights of 16 and $21 \mathrm{ft}$; one pass was made at each height on a 90-deg heading (gage line to pilot's left) and one pass at each height on a $270-$ deg heading (gage line to pilot's right). The data obtained are shown in table 3 . OH-6A helicopter

13. Lift-off tests. Tests were made with the $0 H-6 \mathrm{~A}$ during simulated lift-offs, and data were recorded at helicopter rotations of 30deg increments. These data are shown in table 4.

14. Hover tests. Tests were conducted with the helicopter hovering $34 \mathrm{ft}$ above a given point on the gage line. Data were recorded at each 90-deg increment of rotation. No appreciable velocity was recorded at this test condition as can be seen in table 5 .

15. Fly-by tests. Tests were conducted as the OH-6A flew over a given point on the gage line and perpendicular to it at a rotor height of $14 \mathrm{ft}$ above the surface. Velocity sensors indicated area coverage of downwash was relatively small as can be seen in table 6 . AH-IG helicopter

16. Lift-off tests. Tests were made with the AH-IG positioned with the rotor hub over a given point on the gage line. Recordings of ground-wash velocities were made as the skids cleared the pavement. These data are shown in table 7 .

17. Hover tests. Tests with the AH-IG hovering over a given point on the gage line were made with the rotor $44 \mathrm{ft}$ above the ground surface. As the aircraft rotated over this point, data were recorded for various headings as shown in table 8 .

18. Fly-by tests. In the $f l y$-by tests data were recorded as the helicopter flew slowly over the specified point on the gage line at a rotor height of $22 \mathrm{ft}$. These data are shown in table 9.

UH-1H helicopter

19. Lift-off tests. Tests were made with the UH-1H positioned on a given point on the gage line. Recordings of the ground-wash velocities were made just as the aircraft cleared the pavement, duplicating lift-off conditions. These data are shown in table 10.

20. Hover tests. No hover tests were conducted with the $\mathrm{UH}-1 \mathrm{H}$ 
as its flying time available for these tests was limited because of its special flight mission assignment.

21. Fly-by tests. Data for the fly-by tests were obtained as the $\mathrm{UH}-1 \mathrm{H}$ flew over a given point on the gage line at a rotor height of 20 ft above the ground surface. These data are shown in table 11. UH-IM helicopter

22. Lift-off tests. Tests were made with the UH-IM by placing its rotor hub over a given point on the gage line and recording velocity data as the aircraft's skids cleared the paved surface, duplicating lift-off. The aircraft rotated in 30-deg increments, repeating the lift-off operation until full rotation was achieved. Data obtained are shown in table 12 .

23. Hover tests. Tests were made with the UH-IM hovering over a given point on the gage line at rotor heights of 44 and $66 \mathrm{ft}$ above the paved surface. Data were recorded at 90-deg rotation increments. These data are shown in table 13 .

24. Fly-by tests. No fly-by tests were made with the UH-IM because of the limited time available.

CH-47 helicopter

25. Lift-off tests. Tests were made with the $\mathrm{CH}-47$ positioned so that a point equidistant between its rotor hubs was over a given point on the gage line. Data were recorded as the aircraft lifted off the pavement. The aircraft was rotated in 90-deg increments for each succeeding test and the data obtained are shown in table 14.

26. Hover tests. Tests were conducted with the $\mathrm{CH}-47$ hovering at rotor heights of 50 and $90 \mathrm{ft}$ above the pavement and centered over a given point above the gage line. Data were recorded as the aireraft rotated in 30-deg increments and are shown in table 15.

27. Fly-by tests. Fly-by tests were not made because of the limited size of the test area and large size of the $\mathrm{CH}-47$. $\mathrm{CH}-54$ helicopter

28. Tests were made utilizing the $\mathrm{CH}-54$ helicopter at Ft. Rucker, Ala. The instrumentation layout was the same as described previously herein for the tests at the Apalachicola test site. The weights of the 
CH-54 aircraft, which varied during the test series because of fuel consumption, are shown in the data tables.

29. Lift-off tests. Lift-off tests were simulated by hovering the aircraft just off the munway surface, then lifting it upward and rotating $30 \mathrm{deg}$ into position for the next test. This procedure was selected by the pilot of the aircraft so as to reduce flying time and pilot fatigue. Two series of lift-off tests were made at gross weights of 29,400 and $39,800 \mathrm{lb}$. Lift-off tests could not be conducted for the 47,000-1b maximum gross weight condition because the cargo load extended below the aircraft about $15 \mathrm{ft}$. The lift-off velocity data obtained are shown in table 16.

30. Hover tests. Three series of tests were made with the $\mathrm{CH}-54$ hovering $40 \mathrm{ft}$ above a given point on the gage line, the variable in the tests being the gross weights, which were $28,600,38,000$, and 47,000 Ib. During each series the aircraft was rotated in 30-deg increments. Data obtained are presented in table 17.

31. Other test series were run with the $\mathrm{CH}-54$ hovering $80 \mathrm{ft}$ over a given point on the gage line. Aircraft gross weights for these tests were $27,400,38,000$, and $45,000 \mathrm{lb}$. Data for this series of tests are given in table 18.

32. Fly-by tests. A total of six fly-by tests were made with the $\mathrm{CH}-54$ flying over a given point on the gage line. The aircraft was flown at a slow, steady speed with the rotor $28 \mathrm{ft}$ above the ground surface. Gross aircraft weights were $27,400,38,000$, and 45,000 lb. Data for the fly-by tests are shown in table 19. 
Table 1

Downwash Velocities, OH-58A lift-off Tests

Helicopter Gross Weight, 3000 Ib; Disc Ioad, 3.26 psf;

Rotor Height Above Ground, $10 \mathrm{ft}$

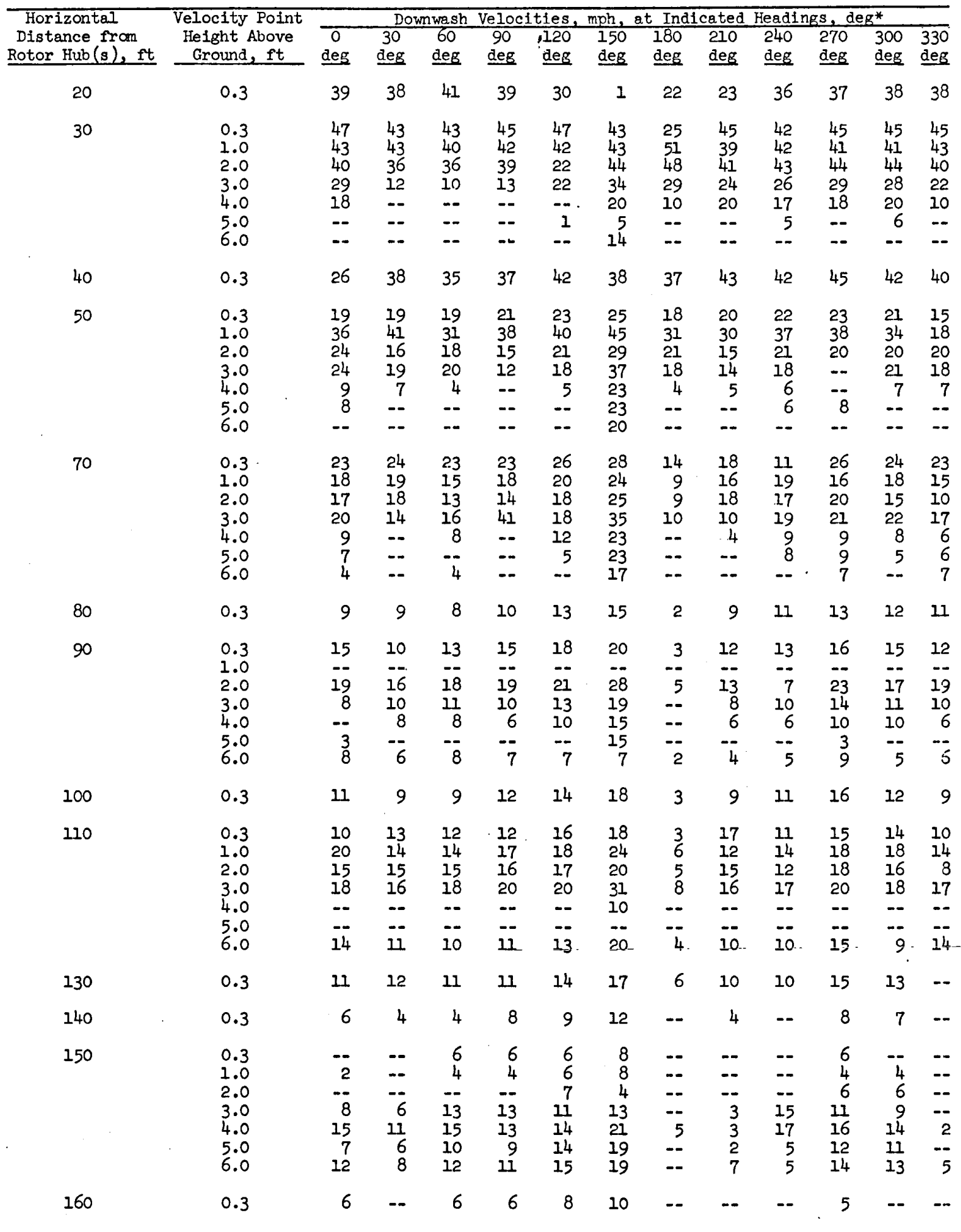

* Heading of helicopter nose relative to line of measurement points. Helicopter at heading of zero degrees faces all measurement points. 


\begin{tabular}{|c|c|c|c|c|c|c|c|c|c|c|c|c|c|c|c|c|c|c|}
\hline \multirow{3}{*}{$\begin{array}{c}\text { Horizontal } \\
\text { Distance from } \\
\text { Rotor Hub(s) ft } \\
\end{array}$} & \multirow{3}{*}{$\begin{array}{l}\text { Velocity Point } \\
\text { Height Above } \\
\text { Ground, ft } \\
\end{array}$} & \multicolumn{17}{|c|}{$\begin{array}{l}\text { Dowmwash Velocities, mph, at Indicated Rotor Helghts } \\
\text { Above Ground, } \mathrm{ft} \text {, and Headings, deg* }\end{array}$} \\
\hline & & \multicolumn{4}{|c|}{$35 \mathrm{ft}$} & \multicolumn{11}{|c|}{$\begin{array}{lllllllllll}0 & 30 & 60 & 90 & 120 & 130 & 180 & 210 & 240 & 27\end{array}$} & \multirow[b]{2}{*}{$\begin{array}{l}300 \\
\text { deg }\end{array}$} & \multirow[b]{2}{*}{$\begin{array}{l}330 \\
\text { deg }\end{array}$} \\
\hline & & $\begin{array}{c}0 \\
\text { deg }\end{array}$ & $\begin{array}{c}90 \\
\text { deg }\end{array}$ & $\begin{array}{l}180 \\
\text { deg }\end{array}$ & $\begin{array}{l}270 \\
\text { deg }\end{array}$ & $\begin{array}{c}0 \\
\text { deg }\end{array}$ & $\begin{array}{r}30 \\
\text { deg }\end{array}$ & $\begin{array}{r}60 \\
\text { deg }\end{array}$ & 90 & $\begin{array}{l}12 \\
\text { de }\end{array}$ & & $\begin{array}{l}150 \\
\text { deg }\end{array}$ & $\begin{array}{l}180 \\
\text { deg }\end{array}$ & $\begin{array}{l}210 \\
\text { deg }\end{array}$ & $\begin{array}{l}240 \\
\text { deg }\end{array}$ & $\begin{array}{l}270 \\
\text { deg }\end{array}$ & & \\
\hline 20 & 0.3 & 33 & 51 & 42 & 49 & 49 & 44 & 51 & 5 & 5 & & 56 & 49 & 49 & 50 & 55 & 51 & 45 \\
\hline 30 & $\begin{array}{l}0.3 \\
1.0 \\
2.0 \\
3.0 \\
4.0 \\
5.0 \\
6.0\end{array}$ & $\begin{array}{l}34 \\
28 \\
35 \\
17 \\
17 \\
11 \\
--\end{array}$ & $\begin{array}{r}44 \\
33 \\
33 \\
15 \\
13 \\
5 \\
-\end{array}$ & $\begin{array}{l}40 \\
29 \\
30 \\
17 \\
23 \\
15 \\
--\end{array}$ & $\begin{array}{r}34 \\
32 \\
25 \\
6 \\
-- \\
-- \\
--\end{array}$ & $\begin{array}{r}45 \\
37 \\
28 \\
8 \\
18 \\
10 \\
5\end{array}$ & $\begin{array}{r}54 \\
39 \\
31 \\
5 \\
- \\
- \\
--\end{array}$ & $\begin{array}{r}45 \\
43 \\
35 \\
15 \\
-- \\
--\end{array}$ & $\begin{array}{r}51 \\
40 \\
36 \\
11 \\
- \\
--\end{array}$ & $\begin{array}{l}5 \\
3 \\
3 \\
1 \\
2 \\
1\end{array}$ & & $\begin{array}{l}54 \\
39 \\
43 \\
28 \\
29 \\
25 \\
16\end{array}$ & $\begin{array}{l}44 \\
39 \\
45 \\
31 \\
40 \\
29 \\
16\end{array}$ & $\begin{array}{r}48 \\
30 \\
20 \\
5 \\
-- \\
-- \\
--\end{array}$ & $\begin{array}{r}50 \\
31 \\
26 \\
5 \\
5 \\
--\end{array}$ & $\begin{array}{r}47 \\
39 \\
34 \\
13 \\
7 \\
-- \\
--\end{array}$ & $\begin{array}{r}46 \\
39 \\
33 \\
8 \\
9 \\
-- \\
--\end{array}$ & $\begin{array}{r}50 \\
39 \\
36 \\
12 \\
7 \\
-- \\
--\end{array}$ \\
\hline 40 & 0.3 & 37 & 34 & 35 & 30 & 32 & 25 & 35 & 37 & 3 & & 31 & 26 & 34 & 33 & 40 & 38 & 43 \\
\hline 50 & $\begin{array}{l}0.3 \\
1.0 \\
2.0 \\
3.0 \\
4.0 \\
5.0 \\
6.0\end{array}$ & $\begin{array}{c}13 \\
24 \\
14 \\
17 \\
12 \\
--\end{array}$ & $\begin{array}{r}17 \\
27 \\
20 \\
14 \\
14 \\
16 \\
6\end{array}$ & $\begin{array}{r}15 \\
22 \\
13 \\
20 \\
10 \\
13 \\
8\end{array}$ & $\begin{array}{r}15 \\
21 \\
13 \\
15 \\
7 \\
33 \\
--\end{array}$ & $\begin{array}{r}18 \\
26 \\
13 \\
10 \\
7 \\
1 \\
--\end{array}$ & $\begin{array}{r}12 \\
22 \\
14 \\
15 \\
3 \\
-- \\
--\end{array}$ & $\begin{array}{r}17 \\
33 \\
20 \\
20 \\
8\end{array}$ & $\begin{array}{l}1 \\
2 \\
1 \\
1 \\
3\end{array}$ & $\begin{array}{l}1 \\
2 \\
1 \\
1 \\
1 \\
1\end{array}$ & & $\begin{array}{l}-- \\
-- \\
-- \\
-- \\
-- \\
--\end{array}$ & $\begin{array}{r}-- \\
-- \\
4 \\
5 \\
6 \\
5\end{array}$ & $\begin{array}{r}8 \\
12 \\
9 \\
-- \\
--\end{array}$ & $\begin{array}{r}11 \\
20 \\
10 \\
18 \\
6 \\
\ldots \\
\ldots\end{array}$ & $\begin{array}{r}16 \\
24 \\
18 \\
19 \\
6 \\
\ldots \\
--\end{array}$ & $\begin{array}{r}-- \\
12 \\
8 \\
6 \\
-- \\
-- \\
--\end{array}$ & $\begin{array}{c}26 \\
22 \\
19 \\
18 \\
6 \\
-- \\
--\end{array}$ \\
\hline 60 & 0.3 & $-\therefore$ & 15 & - & - & 11 & 15 & $\varepsilon$ & 17 & 1 & & -- & -- & -- & -. & -- & -- & -- \\
\hline 70 & $\begin{array}{l}0.3 \\
1.0 \\
2.0 \\
3.0 \\
4.0 \\
5.0 \\
6.0\end{array}$ & $\begin{array}{l}-- \\
-- \\
-- \\
-- \\
-- \\
--\end{array}$ & $\begin{array}{r}18 \\
11 \\
10 \\
12 \\
7 \\
9 \\
4\end{array}$ & $\begin{array}{l}-- \\
=- \\
=- \\
-- \\
=-\end{array}$ & $\begin{array}{l}-- \\
-- \\
-- \\
-- \\
-\end{array}$ & $\begin{array}{l}-- \\
-- \\
-- \\
-- \\
-- \\
--\end{array}$ & $\begin{array}{r}18 \\
13 \\
12 \\
13 \\
6 \\
--\end{array}$ & $\begin{array}{l}17 \\
13 \\
\therefore- \\
\therefore- \\
--\end{array}$ & $\begin{array}{c}15 \\
11 \\
-- \\
--\end{array}$ & 1 & & $\begin{array}{l}-- \\
-- \\
-- \\
-- \\
-- \\
--\end{array}$ & $\begin{array}{l}-. \\
-- \\
-- \\
-- \\
--\end{array}$ & $\begin{array}{l}-- \\
-- \\
-- \\
-- \\
-- \\
--\end{array}$ & $\begin{array}{l}-- \\
-- \\
-- \\
-- \\
-- \\
--\end{array}$ & $\begin{array}{l}-- \\
-- \\
-- \\
-- \\
-- \\
--\end{array}$ & $\begin{array}{l}-- \\
-- \\
-- \\
-- \\
-\end{array}$ & $\begin{array}{l}-- \\
-- \\
-- \\
-- \\
-- \\
--\end{array}$ \\
\hline 80 & 0.3 & -- & -. & -- & -- & - & -- & $\cdots$ & -- & -- & & 5 & 9 & 7 & -- & -- & -- & -- \\
\hline 90 & $\begin{array}{l}0.3 \\
1.0 \\
2.0 \\
3.0 \\
4.0 \\
5.0 \\
6.0\end{array}$ & $\begin{array}{l}-- \\
\because- \\
-- \\
\because- \\
-\therefore \\
--\end{array}$ & $\begin{array}{l}-- \\
-- \\
-- \\
-- \\
--\end{array}$ & $\begin{array}{l}-- \\
-- \\
-- \\
-- \\
-- \\
--\end{array}$ & $\begin{array}{l}-- \\
-- \\
-- \\
-- \\
-- \\
--\end{array}$ & $\begin{array}{r}- \\
2 \\
5 \\
5 \\
2 \\
7 \\
1\end{array}$ & $\begin{array}{l}-- \\
-- \\
-- \\
-- \\
--\end{array}$ & $\begin{array}{l}-- \\
-- \\
-- \\
-- \\
-- \\
--\end{array}$ & \begin{tabular}{l}
-- \\
-- \\
\hdashline- \\
-- \\
--
\end{tabular} & 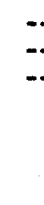 & & $\begin{array}{c}-- \\
-- \\
8 \\
9 \\
8 \\
7 \\
1\end{array}$ & $\begin{array}{r}6 \\
3 \\
13 \\
12 \\
8 \\
10 \\
4\end{array}$ & $\begin{array}{r}-- \\
-8 \\
8 \\
-8 \\
3\end{array}$ & $\begin{array}{r}-- \\
-- \\
3 \\
5 \\
-- \\
-- \\
--\end{array}$ & $\begin{array}{r}5 \\
-- \\
17 \\
19 \\
11 \\
16 \\
8\end{array}$ & $\begin{array}{l}-- \\
-- \\
-- \\
-- \\
-8 \\
1\end{array}$ & $\begin{array}{r}-- \\
-- \\
7 \\
5 \\
-- \\
--\end{array}$ \\
\hline 100 & 0.3 & -- & -- & -- & -- & -- & -- & -- & -- & - & & -- & 6 & - & -- & 6 & $\cdots$ & 3 \\
\hline 110 & $\begin{array}{l}0.3 \\
1.0 \\
2.0 \\
3.0 \\
4.0 \\
5.0 \\
6.0\end{array}$ & $\begin{array}{l}-- \\
-- \\
-- \\
-- \\
-- \\
--\end{array}$ & $\begin{array}{l}-- \\
-- \\
-- \\
-- \\
-- \\
--\end{array}$ & $\begin{array}{l}-- \\
-- \\
-- \\
-- \\
--\end{array}$ & $\begin{array}{l}-- \\
-- \\
-- \\
-- \\
-- \\
--\end{array}$ & $\begin{array}{l}-- \\
-- \\
\overline{-} \\
-- \\
-- \\
-\end{array}$ & $\begin{array}{l}-- \\
-- \\
-- \\
-- \\
--\end{array}$ & $\begin{array}{l}-- \\
-- \\
\because- \\
\because- \\
--\end{array}$ & $\begin{array}{l}-- \\
-- \\
-- \\
-- \\
--\end{array}$ & $\begin{array}{l}- \\
- \\
-\end{array}$ & & $\begin{array}{l}-- \\
-- \\
- \\
- \\
2 \\
7 \\
--\end{array}$ & $\begin{array}{r}2 \\
5 \\
4 \\
3 \\
10 \\
8 \\
6\end{array}$ & $\begin{array}{l}-- \\
-- \\
-- \\
-- \\
--\end{array}$ & $\begin{array}{l}-- \\
-- \\
-- \\
- \\
3 \\
--\end{array}$ & $\begin{array}{r}7 \\
10 \\
8 \\
8 \\
17 \\
18 \\
9\end{array}$ & $\begin{array}{r}2 \\
2 \\
-- \\
-- \\
-- \\
--\end{array}$ & $\begin{array}{l}-- \\
-- \\
-- \\
- \\
8 \\
7 \\
3\end{array}$ \\
\hline 120 & 0.3 & -- & - & -- & -- & 3 & -. & -- & -- & & 3 & -- & 7 & 3 & 1 & 12 & 3 & 5 \\
\hline .140 & .0 .3 & -- & - & -- & -- & 4 & $=-$ & - & -- & - & & - & 5 & - & -- & 8 & 2 & 2 \\
\hline 150 & $\begin{array}{l}0.3 \\
1.0 \\
2.0 \\
3.0 \\
4.0 \\
5.0 \\
6.0\end{array}$ & $\begin{array}{l}-- \\
-- \\
-- \\
-- \\
-- \\
--\end{array}$ & $\begin{array}{l}-- \\
-- \\
-- \\
-- \\
--\end{array}$ & $\begin{array}{l}-- \\
-- \\
-- \\
-- \\
--\end{array}$ & $\begin{array}{l}-- \\
-- \\
-- \\
-- \\
-- \\
--\end{array}$ & $\begin{array}{r}9 \\
9 \\
\text { 11 } \\
-- \\
- \\
0\end{array}$ & $\begin{array}{r}-- \\
-- \\
-- \\
-- \\
\end{array}$ & $\begin{array}{l}-- \\
-- \\
-- \\
-- \\
--\end{array}$ & $\begin{array}{l}-- \\
-- \\
-- \\
-- \\
--\end{array}$ & $\begin{array}{l}1 \\
1 \\
-1 \\
1\end{array}$ & & $\begin{array}{r}8 \\
10 \\
14 \\
-- \\
-- \\
--\end{array}$ & $\begin{array}{r}12 \\
12 \\
16 \\
2 \\
-- \\
10 \\
--\end{array}$ & $\begin{array}{l}-- \\
- \\
- \\
-- \\
-- \\
-\end{array}$ & $\begin{array}{r}4 \\
6 \\
5 \\
-- \\
-- \\
-- \\
--\end{array}$ & $\begin{array}{r}12 \\
13 \\
14 \\
3 \\
1 \\
11 \\
2\end{array}$ & $\begin{array}{r}3 \\
5 \\
7 \\
-- \\
-- \\
-- \\
--\end{array}$ & $\begin{array}{r}-- \\
8 \\
9 \\
-- \\
-- \\
--\end{array}$ \\
\hline 160 & 0.3 & -- & -- & -- & -- & 1 & - & -- & - & & . & -- & - & -. & -- & 3 & -. & -- \\
\hline 170 & 0.3 & -- & -- & $\cdots$ & - & 7 & $\ldots$ & $\cdots$ & - & & 5 & 5 & 7 & -. & - & 6 & -- & 4 \\
\hline 190 & 0.3 & -- & -- & $\ldots$ & -- & 9 & -- & - & - & & 5 & 4 & 8 & - & -- & 10 & 5 & 7 \\
\hline
\end{tabular}


Table 3

Downwash Velocities, OH-58A Fly-By Tests

Helicopter Gross Weight, 3200 and $3000 \mathrm{Ib}$; Disc Load, 3.07 psf;

Rotor Heights Above Ground, 16 and $21 \mathrm{ft}$

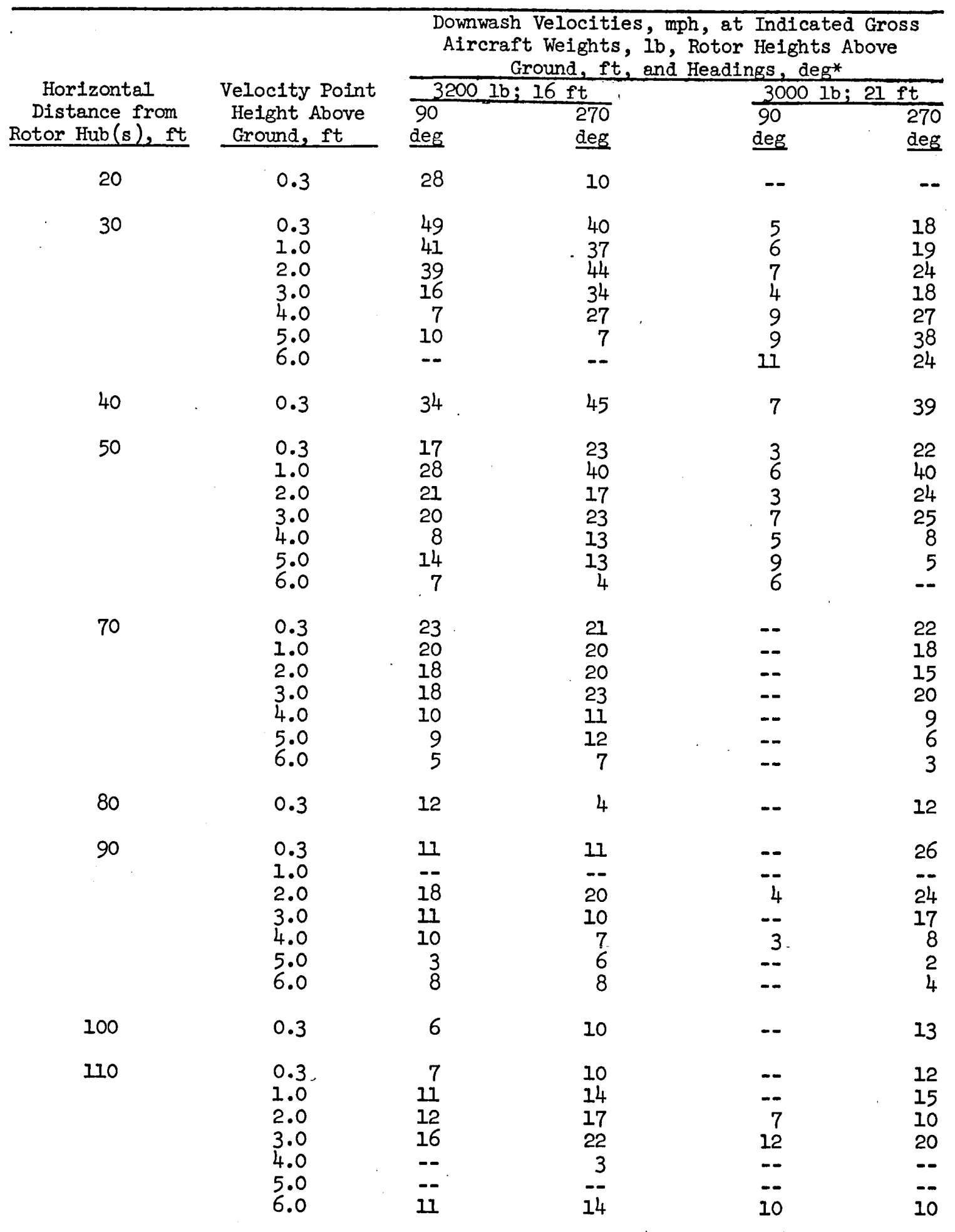

* Heading of helicopter nose relative to line of measurement points. Helicopter at heading of zero degrees faces all measurement points. 
Table 4

Downwash Velocities, $\mathrm{OH}-6 \mathrm{~A}$ Lift-off Tests

Hellcopter Gross Welght, 1800 Ib; Disc Load, 3.31 psf;

Rotor Height Above Ground, $10 \mathrm{ft}$

\begin{tabular}{|c|c|c|c|c|c|c|c|c|c|c|c|c|c|}
\hline Horizontal & Velocity Point & & & awas? & Velor & Eies, & $\mathrm{mph}$, & Ind & cated & eadi & $8 \mathrm{~s}, \mathrm{~d}$ & & \\
\hline $\begin{array}{l}\text { Distance from } \\
\text { Rotor Hub }(\mathrm{s}), \mathrm{ft}\end{array}$ & $\begin{array}{l}\text { Height Above } \\
\text { Ground, ft } \\
\end{array}$ & $\begin{array}{l}0 \\
\text { deg }\end{array}$ & $\begin{array}{l}30 \\
\text { deg } \\
\end{array}$ & $\begin{array}{l}60 \\
\text { deg } \\
\end{array}$ & $\begin{array}{r}90 \\
\text { deg }\end{array}$ & $\begin{array}{l}120 \\
\text { deg }\end{array}$ & $\begin{array}{l}150 \\
\text { deg }\end{array}$ & $\begin{array}{l}180 \\
\text { deg } \\
\end{array}$ & $\begin{array}{l}210 \\
\text { deg } \\
\end{array}$ & $\begin{array}{l}240 \\
\text { deg }\end{array}$ & $\begin{array}{l}270 \\
\text { deg } \\
\end{array}$ & $\begin{array}{l}300 \\
\text { deg }\end{array}$ & $\begin{array}{l}330 \\
\text { deg } \\
\end{array}$ \\
\hline 20 & 0.3 & 37 & 44 & 48 & 46 & 48 & 52 & 48 & $53^{\circ}$ & 45 & 47 & 46 & 45 \\
\hline 30 & $\begin{array}{l}0.3 \\
1.0 \\
2.0 \\
3.0 \\
4.0 \\
5.0 \\
6.0\end{array}$ & $\begin{array}{r}27 \\
14 \\
19 \\
5 \\
10 \\
-- \\
--\end{array}$ & $\begin{array}{r}31 \\
14 \\
18 \\
10 \\
1 \\
3 \\
--\end{array}$ & $\begin{array}{r}28 \\
20 \\
16 \\
2 \\
-- \\
-- \\
--\end{array}$ & $\begin{array}{r}33 \\
20 \\
19 \\
4 \\
-- \\
-- \\
--\end{array}$ & $\begin{array}{r}29 \\
14 \\
15 \\
3 \\
5 \\
-- \\
--\end{array}$ & $\begin{array}{r}37 \\
25 \\
24 \\
10 \\
8 \\
-- \\
--\end{array}$ & $\begin{array}{l}41 \\
29 \\
35 \\
14 \\
14 \\
10 \\
--\end{array}$ & $\begin{array}{r}39 \\
25 \\
22 \\
5 \\
5 \\
-- \\
--\end{array}$ & $\begin{array}{r}31 \\
20 \\
24 \\
4 \\
-- \\
-- \\
--\end{array}$ & $\begin{array}{r}40 \\
29 \\
25 \\
6 \\
6 \\
-- \\
3\end{array}$ & $\begin{array}{r}34 \\
23 \\
24 \\
4 \\
-- \\
-- \\
--\end{array}$ & $\begin{array}{r}33 \\
19 \\
16 \\
2 \\
-- \\
-- \\
--\end{array}$ \\
\hline 40 & 0.3 & -- & -- & 23 & 23 & $2 I$ & 18 & 23 & 7 & 27 & 13 & 23 & 23 \\
\hline 50 & $\begin{array}{l}0.3 \\
1.0 \\
2.0 \\
3.0 \\
4.0 \\
5.0 \\
6.0\end{array}$ & $\begin{array}{l}-- \\
-\therefore \\
-- \\
-- \\
--\end{array}$ & $\begin{array}{l}-- \\
-- \\
-- \\
-- \\
-- \\
--\end{array}$ & $\begin{array}{l}1 \\
2 \\
4 \\
1 \\
1 \\
4 \\
2\end{array}$ & $\begin{array}{r}13 \\
20 \\
14 \\
14 \\
6 \\
- \\
-\end{array}$ & $\begin{array}{r}3 \\
12 \\
6 \\
-- \\
-- \\
1 \\
--\end{array}$ & $\begin{array}{l}-- \\
-- \\
-- \\
-- \\
-- \\
1\end{array}$ & $\begin{array}{l}-- \\
-- \\
-- \\
-- \\
- \\
3\end{array}$ & $\begin{array}{l}-- \\
- \\
- \\
-- \\
-- \\
-- \\
--\end{array}$ & $\begin{array}{l}-- \\
-- \\
-- \\
-- \\
-- \\
--\end{array}$ & $\begin{array}{l}-- \\
-- \\
-- \\
-- \\
-- \\
-- \\
--\end{array}$ & $\begin{array}{r}10 \\
18 \\
10 \\
15 \\
7 \\
6 \\
--\end{array}$ & $\begin{array}{r}6 \\
11 \\
9 \\
10 \\
2 \\
4 \\
--\end{array}$ \\
\hline 60 & 0.3 & -- & -- & -- & 14 & -- & -- & -- & -- & -- & -- & -- & $I$ \\
\hline 70 & $\begin{array}{l}0.3 \\
1.0 \\
2.0 \\
3.0 \\
4.0 \\
5.0 \\
6.0\end{array}$ & $\begin{array}{l}-- \\
-- \\
-- \\
-- \\
-- \\
--\end{array}$ & $\begin{array}{l}-- \\
=- \\
-- \\
-- \\
--\end{array}$ & $\begin{array}{l}-- \\
-- \\
-- \\
-- \\
-- \\
--\end{array}$ & $\begin{array}{r}11 \\
8 \\
5 \\
3 \\
-- \\
-- \\
--\end{array}$ & $\begin{array}{l}-- \\
-- \\
-- \\
-- \\
--\end{array}$ & $\begin{array}{c}1 \\
1 \\
2 \\
-- \\
-- \\
--\end{array}$ & $\begin{array}{l}-- \\
-- \\
-- \\
-- \\
-- \\
--\end{array}$ & $\begin{array}{l}-- \\
-- \\
-- \\
-- \\
-- \\
--\end{array}$ & $\begin{array}{l}-- \\
-- \\
-- \\
-- \\
-- \\
--\end{array}$ & $\begin{array}{l}-- \\
-- \\
-- \\
-- \\
-- \\
--\end{array}$ & $\begin{array}{l}-- \\
-- \\
-- \\
-- \\
-- \\
--\end{array}$ & $\begin{array}{l}-- \\
-- \\
=- \\
-- \\
-- \\
--\end{array}$ \\
\hline 80 & 0.3 & -- & -- & 3 & -- & -- & 7 & -- & 5 & -- & 8 & -- & -- \\
\hline 90 & $\begin{array}{l}0.3 \\
1.0 \\
2.0 \\
3.0 \\
4.0 \\
5.0 \\
6.0\end{array}$ & $\begin{array}{c}-- \\
-- \\
-- \\
-\overline{6} \\
--\end{array}$ & $\begin{array}{r}-- \\
-- \\
3 \\
3 \\
-- \\
--\end{array}$ & $\begin{array}{r}-- \\
-- \\
10 \\
9 \\
6 \\
8 \\
1\end{array}$ & $\begin{array}{l}- \\
- \\
-- \\
- \\
-- \\
--\end{array}$ & $\begin{array}{l}-- \\
-- \\
-- \\
-- \\
-- \\
--\end{array}$ & $\begin{array}{r}-- \\
-- \\
10 \\
9 \\
5 \\
9 \\
2\end{array}$ & $\begin{array}{r}2 \\
-- \\
-- \\
-- \\
1 \\
--\end{array}$ & $\begin{array}{l}- \\
4 \\
7 \\
8 \\
6 \\
6 \\
1\end{array}$ & $\begin{array}{c}4 \\
-- \\
-- \\
-- \\
-- \\
--\end{array}$ & $\begin{array}{r}-- \\
-- \\
13 \\
13 \\
8 \\
10 \\
4\end{array}$ & $\begin{array}{l}-- \\
-- \\
-- \\
-- \\
-- \\
--\end{array}$ & $\begin{array}{l}-- \\
-- \\
-- \\
-- \\
-- \\
2\end{array}$ \\
\hline 100 & 0.3 & -- & -- & -- & -- & -- & 3 & -- & -- & -- & 5 & - & -- \\
\hline 110 & $\begin{array}{l}0.3 \\
1.0 \\
2.0 \\
3.0 \\
4.0 \\
5.0 \\
6.0\end{array}$ & $\begin{array}{l}-- \\
-- \\
-- \\
-- \\
-- \\
--\end{array}$ & $\begin{array}{l}-- \\
-- \\
-- \\
-- \\
-- \\
--\end{array}$ & $\begin{array}{l}-- \\
- \\
- \\
- \\
- \\
-\end{array}$ & $\begin{array}{l}-- \\
- \\
-- \\
- \\
- \\
--\end{array}$ & $\begin{array}{r}-- \\
-- \\
-5 \\
-- \\
-- \\
--\end{array}$ & $\begin{array}{l}-- \\
- \\
-- \\
-6 \\
5 \\
7\end{array}$ & $\begin{array}{r}-- \\
2 \\
2 \\
-- \\
-- \\
-- \\
--\end{array}$ & $\begin{array}{l}=- \\
=- \\
-- \\
-- \\
-- \\
--\end{array}$ & $\begin{array}{r}-- \\
-- \\
-- \\
4 \\
3 \\
--\end{array}$ & $\begin{array}{r}- \\
2 \\
-- \\
-- \\
8 \\
8 \\
--\end{array}$ & $\begin{array}{r}-- \\
2 \\
2 \\
3 \\
4 \\
-- \\
--\end{array}$ & $\begin{array}{l}-- \\
-- \\
-- \\
-- \\
-- \\
--\end{array}$ \\
\hline 120 & 0.3 & -- & -- & -- & $\cdots$ & - & $-\cdots$ & -- & - & -- & 7 & -- & -- \\
\hline
\end{tabular}


Table 5

Downwash Velocities, $\mathrm{OH}-6 \mathrm{~A}$ Hover Tests

Helicopter Gross Weight, 1800 Ib; Disc Load, 3.31 psf;

Rotor Height Above Ground, $34 \mathrm{ft}$

\begin{tabular}{|c|c|c|c|c|c|}
\hline \multirow{2}{*}{$\begin{array}{l}\text { Horizontal } \\
\text { Distance from } \\
\text { Rotor Hub (s), ft }\end{array}$} & \multirow{2}{*}{$\begin{array}{l}\text { Velocity Point } \\
\text { Height Above } \\
\text { Ground, ft } \\
\end{array}$} & \multicolumn{4}{|c|}{$\begin{array}{l}\text { Downwash Velocities, mph, at } \\
\text { Indicated Headings, deg* }\end{array}$} \\
\hline & & $\begin{array}{c}0 \\
\text { deg }\end{array}$ & 90 & 180 & $\begin{array}{l}270 \\
\text { deg }\end{array}$ \\
\hline 20 & 0.3 & 32 & 35 & 47 & 42 \\
\hline 30 & 0.3 & 19 & 31 & 36 & 33 \\
\hline & 1.0 & 12 & 15 & 19 & 19 \\
\hline & 2.0 & 12 & 16 & 19 & 18 \\
\hline & 3.0 & 3 & 5 & 8 & 3 \\
\hline & 4.0 & 9 & 5 & 11 & -- \\
\hline & 5.0 & 4 & -- & 4 & -- \\
\hline & 6.0 & 5 & -- & 5 & -- \\
\hline 40 & 0.3 & -- & -- & 31 & 22 \\
\hline 50 & 0.3 & -- & -- & 9 & -- \\
\hline & 1.0 & $-\infty$ & -- & 13 & -- \\
\hline & 2.0 & -- & -- & 5 & - \\
\hline & 3.0 & - & -- & 4 & -- \\
\hline & 4.0 & -- & - & -- & -- \\
\hline & 5.0 & -- & -- & -- & $-\infty$ \\
\hline & 6.0 & -- & -- & -. & - \\
\hline
\end{tabular}


Table 6

Downwash Velocities, OH-6A Fly-By Tests

Helicopter Gross Weight, 1800 lb; Disc Load, 3.31 psf;

Rotor Height Above Ground, $14 \mathrm{ft}$

\begin{tabular}{|c|c|c|c|}
\hline \multirow{2}{*}{$\begin{array}{c}\text { Horizontal } \\
\text { Distance from } \\
\text { Rotor Hub }(\mathrm{s}), \mathrm{ft}\end{array}$} & \multirow{2}{*}{$\begin{array}{l}\text { Velocity Point } \\
\text { Height Above } \\
\text { Ground, ft }\end{array}$} & \multicolumn{2}{|c|}{$\begin{array}{c}\text { Downwash Velocities } \\
\text { mph, at Indicated } \\
\text { Headings, deg* }\end{array}$} \\
\hline & & $90 \mathrm{deg}$ & $270 \mathrm{deg}$ \\
\hline 20 & 0.3 & 44 & 36 \\
\hline 30 & 0.3 & 34 & 18 \\
\hline & 1.0 & 21 & 12 \\
\hline & 2.0 & 14 & 10 \\
\hline & 3.0 & -- & 1 \\
\hline & 4.0 & -- & -- \\
\hline & 5.0 & - & -- \\
\hline & 6.0 & - & -- \\
\hline 40 & 0.3 & 21 & 10 \\
\hline 50 & 0.3 & -- & 7 \\
\hline & 1.0 & $-m$ & 5 \\
\hline & 2.0 & - & 5 \\
\hline & 3.0 & - & 4 \\
\hline & 4.0 & - & 1 \\
\hline & 5.0 & - & - \\
\hline & 6.0 & -- & - \\
\hline
\end{tabular}

Heading of helicopter nose relative to line of measurement points. Helicopter at heading of zero degrees faces all measurement points. 
Table 7

Downwash Velocities, AH-1G Lift-Off Tests

Helicopter Gross Weight, 9500 lb; Disc Load, 6.25 psf;

Rotor Height Above Ground, $14 \mathrm{ft}$

\begin{tabular}{|c|c|c|c|c|c|c|c|c|c|c|c|c|c|}
\hline \multirow{3}{*}{$\begin{array}{l}\text { Horizontal } \\
\text { Distance from } \\
\text { Rotor Hub (s), ft }\end{array}$} & \multirow{3}{*}{$\begin{array}{l}\text { Velocity Point } \\
\text { Height Above } \\
\text { Ground, ft } \\
\end{array}$} & \multicolumn{12}{|c|}{ Downwash Velocities, mph, at Indicated Headings, deg* } \\
\hline & & 0 & 30 & 60 & 90 & 120 & 150 & 180 & 210 & 240 & 270 & 300 & 330 \\
\hline & & deg & deg & deg & deg & deg & deg & deg & deg & $\underline{\operatorname{deg}}$ & $\underline{\mathrm{deg}}$ & deg & deg \\
\hline 20 & 0.3 & -- & - & 49 & -- & 29 & 24 & -- & 37 & 16 & 22 & 32 & 30 \\
\hline 30 & $\begin{array}{l}0.3 \\
1.0 \\
2.0 \\
3.0 \\
4.0 \\
5.0 \\
6.0\end{array}$ & $\begin{array}{l}-- \\
-- \\
-- \\
-- \\
--\end{array}$ & $\begin{array}{l}51 \\
=- \\
-- \\
-- \\
-- \\
--\end{array}$ & $\begin{array}{l}72 \\
63 \\
75 \\
59 \\
46 \\
21 \\
--\end{array}$ & $\begin{array}{l}43 \\
-- \\
-- \\
-- \\
-- \\
--\end{array}$ & $\begin{array}{r}75 \\
62 \\
75 \\
59 \\
40 \\
9 \\
--\end{array}$ & $\begin{array}{l}74 \\
69 \\
76 \\
64 \\
52 \\
12 \\
-\end{array}$ & $\begin{array}{l}24 \\
-- \\
-- \\
-- \\
-- \\
--\end{array}$ & $\begin{array}{l}74 \\
57 \\
74 \\
78 \\
75 \\
52 \\
22\end{array}$ & $\begin{array}{l}68 \\
51 \\
70 \\
60 \\
70 \\
64 \\
45\end{array}$ & $\begin{array}{l}62 \\
47 \\
65 \\
62 \\
77 \\
68 \\
41\end{array}$ & $\begin{array}{l}68 \\
51 \\
68 \\
62 \\
70 \\
54 \\
38\end{array}$ & $\begin{array}{l}63 \\
49 \\
70 \\
64 \\
75 \\
63 \\
24\end{array}$ \\
\hline 40 & $\begin{array}{l}0.3 \\
1.0 \\
2.0 \\
3.0 \\
4.0 \\
5.0 \\
6.0\end{array}$ & $\begin{array}{l}-- \\
-- \\
-- \\
-- \\
-- \\
--\end{array}$ & $\begin{array}{r}71 \\
55 \\
72 \\
51 \\
51 \\
29 \\
9\end{array}$ & $\begin{array}{l}67 \\
-- \\
-- \\
-- \\
-- \\
-- \\
--\end{array}$ & $\begin{array}{l}71 \\
62 \\
74 \\
55 \\
33 \\
10 \\
-\end{array}$ & $\begin{array}{l}70 \\
-- \\
-- \\
-- \\
-- \\
--\end{array}$ & $\begin{array}{l}70 \\
-- \\
-- \\
-- \\
-- \\
--\end{array}$ & $\begin{array}{l}60 \\
49 \\
64 \\
43 \\
37 \\
21 \\
15\end{array}$ & $\begin{array}{l}68 \\
-- \\
-- \\
-- \\
-- \\
--\end{array}$ & $\begin{array}{l}74 \\
-- \\
-- \\
-- \\
-- \\
--\end{array}$ & $\begin{array}{l}71 \\
-- \\
-- \\
-- \\
-- \\
-- \\
--\end{array}$ & $\begin{array}{l}72 \\
-- \\
-- \\
-- \\
-- \\
--\end{array}$ & $\begin{array}{l}72 \\
-- \\
-- \\
-- \\
-- \\
--\end{array}$ \\
\hline 50 & $\begin{array}{l}0.3 \\
1.0 \\
2.0 \\
3.0 \\
4.0 \\
5.0 \\
6.0\end{array}$ & $\begin{array}{l}64 \\
-- \\
-- \\
-- \\
-- \\
--\end{array}$ & $\begin{array}{l}63 \\
-- \\
-- \\
-- \\
-- \\
-- \\
--\end{array}$ & $\begin{array}{r}40 \\
70 \\
39 \\
50 \\
22 \\
19 \\
5\end{array}$ & $\begin{array}{c}64 \\
-- \\
-- \\
-- \\
-- \\
--\end{array}$ & $\begin{array}{l}37 \\
65 \\
40 \\
42 \\
16 \\
10 \\
--\end{array}$ & $\begin{array}{r}39 \\
67 \\
41 \\
43 \\
18 \\
22 \\
5\end{array}$ & $\begin{array}{l}57 \\
-- \\
-- \\
-- \\
-- \\
--\end{array}$ & $\begin{array}{l}40 \\
69 \\
31 \\
45 \\
24 \\
28 \\
12\end{array}$ & $\begin{array}{l}49 \\
74 \\
44 \\
55 \\
28 \\
37 \\
18\end{array}$ & $\begin{array}{l}46 \\
74 \\
48 \\
59 \\
33 \\
36 \\
28\end{array}$ & $\begin{array}{l}44 \\
71 \\
46 \\
59 \\
30 \\
28 \\
16\end{array}$ & $\begin{array}{l}49 \\
75 \\
47 \\
51 \\
27 \\
29 \\
14\end{array}$ \\
\hline 60 & $\begin{array}{l}0.3 \\
1.0 \\
2.0 \\
3.0 \\
4.0 \\
5.0 \\
6.0\end{array}$ & $\begin{array}{l}74 \\
49 \\
72 \\
57 \\
50 \\
15 \\
-\end{array}$ & $\begin{array}{l}33 \\
66 \\
35 \\
41 \\
20 \\
21 \\
10\end{array}$ & $\begin{array}{l}-- \\
-- \\
-- \\
-- \\
-- \\
--\end{array}$ & $\begin{array}{r}39 \\
68 \\
44 \\
45 \\
18 \\
18 \\
6\end{array}$ & $\begin{array}{l}-- \\
-- \\
-- \\
-- \\
-- \\
--\end{array}$ & $\begin{array}{l}-- \\
-- \\
-- \\
-- \\
-- \\
--\end{array}$ & $\begin{array}{l}28 \\
43 \\
28 \\
32 \\
15 \\
24 \\
27\end{array}$ & $\begin{array}{l}-- \\
-- \\
-- \\
-- \\
-- \\
--\end{array}$ & $\begin{array}{l}-- \\
-- \\
-- \\
-- \\
-- \\
--\end{array}$ & $\begin{array}{l}-- \\
-- \\
-- \\
-- \\
-- \\
--\end{array}$ & $\begin{array}{l}-- \\
-- \\
-- \\
-- \\
-- \\
--\end{array}$ & $\begin{array}{l}-- \\
-- \\
-- \\
-- \\
-- \\
--\end{array}$ \\
\hline 70 & $\begin{array}{l}0.3 \\
1.0 \\
2.0 \\
3.0 \\
4.0 \\
5.0 \\
6.0\end{array}$ & $\begin{array}{l}63 \\
-- \\
-- \\
-- \\
-- \\
-- \\
--\end{array}$ & $\begin{array}{l}-- \\
-- \\
-- \\
-- \\
-- \\
--\end{array}$ & $\begin{array}{l}44 \\
38 \\
35 \\
47 \\
23 \\
27 \\
15\end{array}$ & $\begin{array}{l}-- \\
-- \\
-- \\
-- \\
-- \\
--\end{array}$ & $\begin{array}{l}45 \\
41 \\
33 \\
38 \\
15 \\
18 \\
13\end{array}$ & $\begin{array}{l}39 \\
31 \\
30 \\
36 \\
14 \\
12 \\
12\end{array}$ & $\begin{array}{l}-- \\
-- \\
-- \\
-- \\
-- \\
--\end{array}$ & $\begin{array}{l}38 \\
37 \\
29 \\
44 \\
19 \\
23 \\
18\end{array}$ & $\begin{array}{l}46 \\
43 \\
39 \\
46 \\
19 \\
25 \\
14\end{array}$ & $\begin{array}{l}51 \\
44 \\
40 \\
50 \\
25 \\
31 \\
20\end{array}$ & $\begin{array}{l}49 \\
46 \\
41 \\
46 \\
24 \\
32 \\
20\end{array}$ & $\begin{array}{l}49 \\
40 \\
33 \\
48 \\
26 \\
31 \\
14\end{array}$ \\
\hline 80 & $\begin{array}{l}0.3 \\
1.0 \\
2.0 \\
3.0 \\
4.0 \\
5.0 \\
6.0\end{array}$ & $\begin{array}{l}34 \\
66 \\
44 \\
50 \\
18 \\
20 \\
--\end{array}$ & $\begin{array}{l}39 \\
40 \\
35 \\
36 \\
17 \\
17 \\
15\end{array}$ & $\begin{array}{l}25 \\
-- \\
-- \\
-- \\
-- \\
--\end{array}$ & $\begin{array}{l}42 \\
36 \\
36 \\
42 \\
18 \\
20 \\
32\end{array}$ & $\begin{array}{l}30 \\
-- \\
-- \\
-- \\
-- \\
--\end{array}$ & $\begin{array}{c}25 \\
-- \\
-- \\
-- \\
--\end{array}$ & $\begin{array}{r}13 \\
21 \\
19 \\
25 \\
13 \\
14 \\
8\end{array}$ & $\begin{array}{l}26 \\
-- \\
-- \\
-- \\
-- \\
--\end{array}$ & $\begin{array}{l}28 \\
-- \\
-- \\
-- \\
-- \\
--\end{array}$ & $\begin{array}{l}36 \\
-- \\
-- \\
-- \\
-- \\
--\end{array}$ & $\begin{array}{l}34 \\
-- \\
-- \\
-- \\
-- \\
--\end{array}$ & $\begin{array}{l}30 \\
-- \\
-- \\
-- \\
-- \\
--\end{array}$ \\
\hline 90 & $\begin{array}{l}0.3 \\
1.0 \\
2.0 \\
3.0 \\
4.0 \\
5.0 \\
6.0\end{array}$ & $\begin{array}{l}-- \\
-- \\
-- \\
-- \\
-- \\
--\end{array}$ & $\begin{array}{l}26 \\
-- \\
-- \\
-- \\
-- \\
--\end{array}$ & $\begin{array}{r}31 \\
38 \\
23 \\
15 \\
9 \\
17\end{array}$ & $\begin{array}{l}27 \\
- \\
-- \\
-- \\
--\end{array}$ & $\begin{array}{r}31 \\
- \\
36 \\
22 \\
15 \\
9 \\
13\end{array}$ & $\begin{array}{l}30 \\
38 \\
21 \\
20 \\
10 \\
13\end{array}$ & $\begin{array}{l}-- \\
-- \\
-- \\
-- \\
-- \\
--\end{array}$ & $\begin{array}{l}28 \\
-1 \\
39 \\
29 \\
19 \\
12 \\
15\end{array}$ & $\begin{array}{l}31 \\
-14 \\
34 \\
22 \\
18 \\
17\end{array}$ & $\begin{array}{l}36 \\
-17 \\
40 \\
26 \\
20 \\
19\end{array}$ & $\begin{array}{l}36 \\
-43 \\
31 \\
18 \\
21 \\
18\end{array}$ & $\begin{array}{l}38 \\
-16 \\
31 \\
20 \\
16 \\
20\end{array}$ \\
\hline & & & & tin & & & & & & & & & \\
\hline
\end{tabular}

* Heading of helicopter nose relative to line of measurement points. Helicopter at heading of zero degrees faces all measurement points. 
Table 7 (Concluded)

\begin{tabular}{|c|c|c|c|c|c|c|c|c|c|c|c|c|c|}
\hline Horizontal & Velocity Point & & DWn we & $\operatorname{sh} \mathrm{Ve}$ & ocit & ies, & $\mathrm{nph}$, & at Ir & dicat & $\mathrm{de}$ & ading & $5, \mathrm{de}$ & \\
\hline Distance from & Height Above & 0 & 30 & 60 & 90 & 120 & 150 & 180 & 210 & 240 & 270 & 300 & 330 \\
\hline Rotor $\mathrm{Hub}(\mathrm{s}), \mathrm{ft}$ & Ground, ft & deg & deg & deg & deg & deg & deg & deg & deg & deg & deg & deg & deg \\
\hline 100 & 0.3 & 39 & 25 & 26 & 28 & $3 I$ & 31 & 16 & 30 & 35 & 33 & 33 & 31 \\
\hline & 1.0 & 37 & $=$ & -- & -- & -- & -- & -- & -- & -- & -- & -- & -- \\
\hline & 2.0 & 32 & 38 & -- & 40 & -- & -- & 22 & -- & -- & -- & -- & -- \\
\hline & 3.0 & 40 & 27 & -- & 28 & - & -- & 14 & - & -- & -- & -- & -- \\
\hline & 4.0 & 19 & 18 & -- & 17 & -- & -- & 12 & -- & -- & -- & -- & -- \\
\hline & 5.0 & 21 & 9 & -- & 11 & -- & - & 3 & -- & -- & -- & -- & -- \\
\hline & 6.0 & 12 & 14 & -- & 14 & -- & -- & 10 & -- & -- & -- & -- & -- \\
\hline 110 & 0.3 & 24 & 24 & 23 & 27 & 28 & 25 & 20 & 25 & 27 & 27 & 26 & 22 \\
\hline & 1.0 & -- & -- & 29 & - & 31 & 30 & -- & 30 & 34 & 33 & 31 & 29 \\
\hline & 2.0 & -- & -- & 30 & - & 23 & 33 & -- & 30 & 32 & 35 & 35 & 32 \\
\hline & 3.0 & - & -- & 40 & -- & 35 & 44 & -- & 39 & 41 & 46 & 43 & 40 \\
\hline & 4.0 & -- & -- & 18 & -- & 12 & 19 & -- & 17 & 18 & 22 & 16 & 16 \\
\hline & 5.0 & -- & $\cdots$ & $\cdots$ & -- & - & $=$ & -- & -- & -- & -- & -- & -- \\
\hline & 6.0 & -- & -- & 31 & -- & 31 & 28 & -- & 34 & 35 & 39 & 30 & 32 \\
\hline 120 & 0.3 & 28 & 20 & 12 & 25 & 9 & 10 & 14 & 8 & 15 & 16 & 14 & 12 \\
\hline & 1.0 & - & 28 & -- & 28 & -- & -- & 17 & -- & -- & -- & -- & -- \\
\hline & 2.0 & 37 & 28 & -- & 29 & -- & -- & 20 & -- & -- & -- & -- & -- \\
\hline & 3.0 & 24 & 37 & -- & 38 & -- & - & 30 & $\cdots$ & -- & - & - & - \\
\hline & 4.0 & 14 & 3 & -- & 12 & -- & -- & -- & -- & $\cdots$ & -- & -- & - \\
\hline & 5.0 & 13 & - & -- & -- & -- & -- & -- & -- & -- & -- & -- & -- \\
\hline & 6.0 & 17 & 24 & -- & 30 & -- & -- & 22 & - & -- & - & -- & -- \\
\hline 130 & 0.3 & 24 & 6 & 22 & 10 & 25 & 25 & -- & 24 & 25 & 30 & 25 & 24 \\
\hline 140 & 0.3 & 20 & 22 & 15 & 22 & 19 & 18 & 5 & 18 & 22 & 23 & 16 & 20 \\
\hline & 1.0 & 25 & -- & -- & $\cdots$ & -- & -- & -- & -- & -- & -- & -- & -- \\
\hline & 2.0 & 26 & 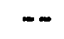 & -- & -- & -- & -- & -- & -- & -- & -- & -- & - \\
\hline & 3.0 & 33 & -- & -- & -- & -- & -- & -- & -- & -- & -- & -- & -- \\
\hline & 4.0 & 7 & -- & -- & -- & -- & - & - & -- & - & -- & -- & $=$ \\
\hline & 5.0 & -- & -- & -- & -- & -- & - & -- & -- & -- & -- & - & -- \\
\hline & 6.0 & 29 & $\cdots$ & -- & -- & $\cdots$ & $\cdots$ & - & - & $\cdots$ & - & -- & -- \\
\hline 150 & 0.3 & 7 & 18 & 17 & 17 & 18 & 17 & 9 & 10 & 19 & 22 & 15 & 18 \\
\hline & 1.0 & -- & -- & 13 & - & 15 & 16 & -- & 12 & 10 & 20 & 12 & 17 \\
\hline & 2.0 & -- & -- & 13 & - & 15 & 16 & -- & 14 & 22 & 20 & 16 & 20 \\
\hline & 3.0 & -- & -- & 16 & $\cdots$ & 19 & 17 & -- & 18 & 21 & 22 & 18 & 19 \\
\hline & 4.0 & -- & -- & 29 & - & 30 & 27 & -- & 31 & 32 & 35 & 31 & 33 \\
\hline & 5.0 & -- & -- & 19 & -- & 22 & 16 & -- & 23 & 23 & 28 & 25 & 26 \\
\hline & 6.0 & -- & -- & 18 & -- & 21 & 16 & -- & 22 & 22 & 26 & 25 & 26 \\
\hline 160 & 0.3 & 22 & 15 & 12 & 18 & 14 & 12 & -- & 11 & 17 & 19 & 12 & 16 \\
\hline & 1.0 & - & 13 & -- & 15 & - & $\cdots$ & 6 & -- & -- & -- & -- & -- \\
\hline & 2.0 & -- & 16 & -- & 20 & -- & -- & 9 & - & -- & -- & -- & -- \\
\hline & 3.0 & -- & 17 & -- & 18 & -- & -- & 17 & -- & -- & -- & -- & -- \\
\hline & 4.0 & -- & 30 & -- & 27 & -- & -- & 22 & -- & -- & -- & - & - \\
\hline & 5.0 & -- & 20 & -- & 19 & -- & -- & 37 & -- & -- & -- & - & -- \\
\hline & 6.0 & -- & 20 & -- & 20 & -- & -- & 14 & -- & $\cdots$ & $\cdots$ & -- & $\cdots$ \\
\hline 170 & 0.3 & 16 & 13 & II & 14 & 11 & 10 & - & 8 & 13 & 17 & 10 & 15 \\
\hline 180 & 0.3 & 13 & 8 & - & 12 & -- & -- & -- & -- & -- & -- & -- & -- \\
\hline & 1.0 & 16 & -- & - & -- & -- & -- & -- & -- & -- & -- & $=$ & -- \\
\hline & 2.0 & 18 & -- & -- & - & -- & -- & -- & -- & -- & - & -- & - \\
\hline & 3.0 & 19 & -- & -- & -- & - & -- & -- & -- & - & -- & -- & -- \\
\hline & 4.0 & 32 & -- & -- & -- & -- & -- & -- & -- & -- & -- & -- & -- \\
\hline & 5.0 & 22 & -- & -- & -- & -- & -- & -- & -- & -- & -- & -- & -- \\
\hline & 6.0 & 20 & -- & -- & -- & -- & -- & -- & -- & -- & -- & - & -- \\
\hline
\end{tabular}


Table 8

Downwash Velocities, AH-1G Hover Tests

Helicopter Gross Weight, 9500 Ib; Disc Load, 6.25 psf; Rotor Height Above Ground, $44 \mathrm{ft}$

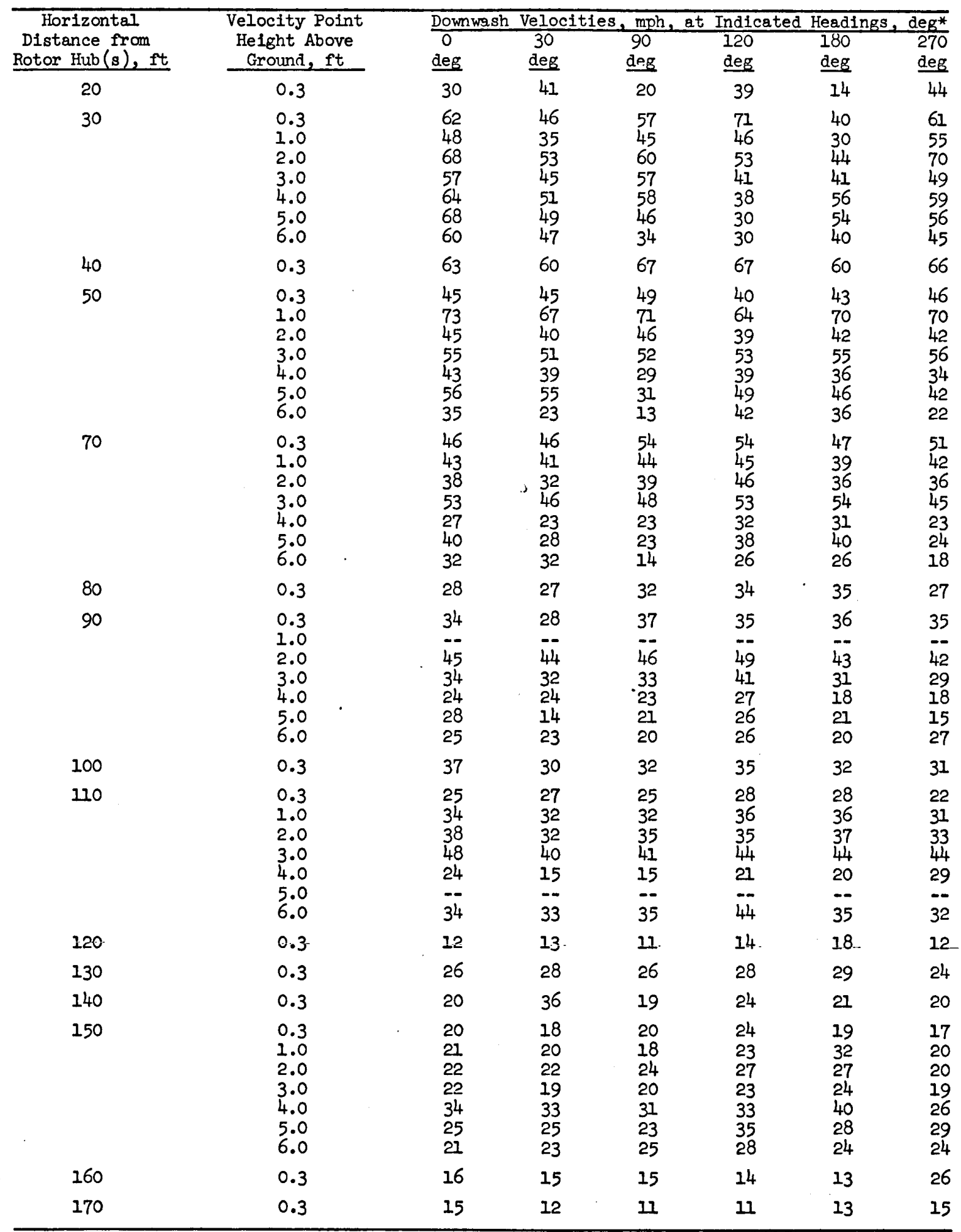

* Heading of hellcopter nose relative to line of measurement points. Helicopter at heading of zero degrees faces all measurement points. 
Table 9

Downwash Velocities, AH-1G Fly-By Tests

Helicopter Gross Weight, 9500 Ib; Disc Load, 6.25 psf;

Rotor Height Above Ground, 22 ft

\begin{tabular}{|c|c|c|c|}
\hline \multirow{2}{*}{$\begin{array}{c}\text { Horizontal } \\
\text { Distance from } \\
\text { Rotor Hub(s), ft }\end{array}$} & \multirow{2}{*}{$\begin{array}{l}\text { Velocity Point } \\
\text { Height Above } \\
\text { Ground, ft } \\
\end{array}$} & \multicolumn{2}{|c|}{$\begin{array}{c}\text { Downwash Velocities, mph, at Indicated } \\
\text { Headings, deg* }\end{array}$} \\
\hline & & $\begin{array}{r}90 \\
\text { deg } \\
\end{array}$ & $\begin{array}{l}270 \\
\text { deg }\end{array}$ \\
\hline 20 & 0.3 & 10 & 21 \\
\hline 30 & 0.3 & 51 & 52 \\
\hline & 1.0 & 36 & 51 \\
\hline & 2.0 & 40 & 59 \\
\hline & 3.0 & 37 & 46 \\
\hline & 4.0 & 43 & 46 \\
\hline & 5.0 & 32 & 26 \\
\hline & 6.0 & 25 & 11 \\
\hline 40 & 0.3 & 48 & 54 \\
\hline 50 & 0.3 & 25 & 32 \\
\hline & 1.0 & 41 & 54 \\
\hline & 2.0 & 26 & 42 \\
\hline & 3.0 & 35 & 45 \\
\hline & 4.0 & 41 & 16 \\
\hline & 5.0 & 24 & 11 \\
\hline & 6.0 & 18 & -- \\
\hline 70 & 0.3 & 30 & 35 \\
\hline & 1.0 & 24 & 36 \\
\hline & 2.0 & 22 & 29 \\
\hline & 3.0 & 30 & 39 \\
\hline & 4.0 & 17 & 13 \\
\hline & 5.0 & 21 & 12 \\
\hline & 6.0 & 16 & 7 \\
\hline 80 & 0.3 & tinued) & 22 \\
\hline
\end{tabular}

* Heading of helicopter nose relative to line of measurement points. Helicopter at heading of zero degrees faces all measurement points. 
Table 9 (Concluded)

\begin{tabular}{|c|c|c|c|}
\hline \multirow{3}{*}{$\begin{array}{c}\text { Horizontal } \\
\text { Distance from } \\
\text { Rotor Hub }(s), \text { ft } \\
\end{array}$} & \multirow{3}{*}{$\begin{array}{l}\text { Velocity Point } \\
\text { Height Above } \\
\text { Ground, ft } \\
\end{array}$} & \multicolumn{2}{|c|}{$\begin{array}{c}\text { Downwash Velocities, mph, at Indicated } \\
\text { Headings, deg } \\
\end{array}$} \\
\hline & & 90 & 270 \\
\hline & & $\underline{\text { deg }}$ & \\
\hline \multirow[t]{7}{*}{90} & 0.3 & 29 & 25 \\
\hline & 1.0 & -- & -- \\
\hline & 2.0 & 24 & 34 \\
\hline & 3.0 & 18 & 34 \\
\hline & 4.0 & 14 & 19 \\
\hline & 5.0 & 11 & 10 \\
\hline & 6.0 & 16 & 11 \\
\hline 100 & 0.3 & 17 & 21 \\
\hline \multirow[t]{7}{*}{110} & 0.3 & 14 & 17 \\
\hline & 1.0 & 19 & 22 \\
\hline & 2.0 & 22 & 27 \\
\hline & 3.0 & 30 & 37 \\
\hline & 4.0 & 7 & 12 \\
\hline & 5.0 & -- & $-\infty$ \\
\hline & 6.0 & 29 & 32 \\
\hline 120 & 0.3 & -- & 5 \\
\hline 130 & 0.3 & 11 & 15 \\
\hline 140 & 0.3 & 4 & 8 \\
\hline \multirow[t]{7}{*}{150} & 0.3 & 3 & 7 \\
\hline & 1.0 & 4 & 12 \\
\hline & 2.0 & 5 & 7 \\
\hline & 3.0 & 10 & 15 \\
\hline & 4.0 & 21 & 25 \\
\hline & 5.0 & 13 & 14 \\
\hline & 6.0 & 15 & 15 \\
\hline 160 & 0.3 & 9 & 9 \\
\hline 170 & 0.3 & 6 & 5 \\
\hline
\end{tabular}


Table 10

Downwash Velocities, UH-1H Lift-Off Tests

Helicopter Gross Weight, 9500 lb; Disc Load, 5.25 psf; Rotor Height Above Ground, $14 \mathrm{ft}$

\begin{tabular}{|c|c|c|c|c|c|c|c|c|c|c|c|}
\hline Horizontal & Velocity Point & & & $\frac{\text { wash }}{60}$ & ociti & $\mathrm{mph}_{2}$ & Indi & d He & $\frac{n g s_{2}}{g_{2}}$ & & \\
\hline $\begin{array}{l}\text { Distance from } \\
\text { Rotor Hub }(\mathrm{s}), \mathrm{ft}\end{array}$ & $\begin{array}{l}\text { Height Above } \\
\text { Ground, } \mathrm{ft} \\
\end{array}$ & $\begin{array}{l}0 \\
\text { deg } \\
\end{array}$ & $\begin{array}{l}0 \\
\mathrm{deg}\end{array}$ & $\begin{array}{l}60 \\
\text { deg } \\
\end{array}$ & $\begin{array}{l}90 \\
\text { deg } \\
\end{array}$ & $\begin{array}{l}120 \\
\text { deg } \\
\end{array}$ & $\begin{array}{l}150 \\
\text { deg }\end{array}$ & $\begin{array}{l}180 \\
\text { deg }\end{array}$ & $\begin{array}{l}210 \\
\text { deg }\end{array}$ & $\begin{array}{l}240 \\
\text { deg }\end{array}$ & $\begin{array}{l}270 \\
\text { deg }\end{array}$ \\
\hline 20 & 0.3 & -- & -- & 19 & 25 & 34 & -- & -- & -- & 23 & 63 \\
\hline 30 & $\begin{array}{l}0.3 \\
1.0 \\
2.0 \\
3.0 \\
4.0 \\
5.0 \\
6.0\end{array}$ & $\begin{array}{l}-- \\
=- \\
=- \\
-- \\
=- \\
--\end{array}$ & $\begin{array}{c}-- \\
-- \\
-- \\
-- \\
--\end{array}$ & $\begin{array}{r}65 \\
59 \\
62 \\
47 \\
35 \\
8 \\
---\end{array}$ & $\begin{array}{l}-- \\
61 \\
63 \\
54 \\
51 \\
35 \\
12\end{array}$ & $\begin{array}{l}57 \\
63 \\
59 \\
53 \\
33 \\
49 \\
50\end{array}$ & $\begin{array}{l}- \\
- \\
- \\
- \\
- \\
-- \\
--\end{array}$ & $\begin{array}{l}-- \\
-- \\
-- \\
-- \\
-- \\
--\end{array}$ & $\begin{array}{l}-- \\
-- \\
-- \\
-- \\
-- \\
--\end{array}$ & $\begin{array}{l}57 \\
53 \\
58 \\
54 \\
54 \\
52 \\
31\end{array}$ & $\begin{array}{l}41 \\
48 \\
51 \\
47 \\
46 \\
31 \\
16\end{array}$ \\
\hline 40 & 0.3 & -- & -- & 62 & 52 & 60 & 64 & 63 & 66 & 64 & 39 \\
\hline 50 & $\begin{array}{l}0.3 \\
1.0 \\
2.0 \\
3.0 \\
4.0 \\
5.0 \\
6.0\end{array}$ & $\begin{array}{l}-- \\
-- \\
=- \\
=- \\
--\end{array}$ & $\begin{array}{l}-- \\
-- \\
-- \\
-- \\
--\end{array}$ & $\begin{array}{l}35 \\
58 \\
32 \\
32 \\
12 \\
-- \\
--\end{array}$ & $\begin{array}{l}-- \\
38 \\
36 \\
22 \\
15 \\
30\end{array}$ & $\begin{array}{l}-- \\
-- \\
39 \\
41 \\
27 \\
30 \\
13\end{array}$ & $\begin{array}{l}61 \\
60 \\
57 \\
40 \\
29 \\
13 \\
--\end{array}$ & $\begin{array}{l}61 \\
62 \\
62 \\
61 \\
66 \\
62 \\
47\end{array}$ & $\begin{array}{r}63 \\
61 \\
58 \\
43 \\
41 \\
21 \\
7\end{array}$ & $\begin{array}{l}-- \\
-- \\
46 \\
21 \\
17 \\
--\end{array}$ & $\begin{array}{l}14 \\
43 \\
30 \\
40 \\
25 \\
36 \\
20\end{array}$ \\
\hline 60 & 0.3 & -- & -- & - & -- & -- & 55 & 47 & 56 & -- & -- \\
\hline 70 & $\begin{array}{l}0.3 \\
1.0 \\
2.0 \\
3.0 \\
4.0 \\
5.0 \\
6.0\end{array}$ & $\begin{array}{l}-- \\
-- \\
-- \\
-- \\
-- \\
--\end{array}$ & $\begin{array}{c}-- \\
-- \\
-- \\
-- \\
-- \\
--\end{array}$ & $\begin{array}{l}34 \\
29 \\
26 \\
35 \\
14 \\
18 \\
12\end{array}$ & $\begin{array}{l}39 \\
35 \\
27 \\
-- \\
-- \\
-- \\
--\end{array}$ & $\begin{array}{l}40 \\
36 \\
34 \\
37 \\
17 \\
18 \\
15\end{array}$ & $\begin{array}{l}35 \\
50 \\
27 \\
22 \\
14 \\
13 \\
--\end{array}$ & $\begin{array}{l}27 \\
56 \\
36 \\
54 \\
39 \\
56 \\
36\end{array}$ & $\begin{array}{l}35 \\
54 \\
34 \\
45 \\
24 \\
25 \\
12\end{array}$ & $\begin{array}{l}44 \\
35 \\
36 \\
37 \\
19 \\
24 \\
--\end{array}$ & $\begin{array}{l}28 \\
26 \\
27 \\
- \\
17 \\
22 \\
20\end{array}$ \\
\hline 80 & 0.3 & $\cdots$ & -- & 24 & 19 & 26 & -- & -- & - & 30 & 17 \\
\hline 90 & $\begin{array}{l}0.3 \\
1.0 \\
2.0 \\
3.0 \\
4.0 \\
5.0 \\
6.0\end{array}$ & $\begin{array}{l}19 \\
-- \\
24 \\
12 \\
20 \\
-- \\
--\end{array}$ & $\begin{array}{l}21 \\
3- \\
31 \\
22 \\
28 \\
-- \\
--\end{array}$ & $\begin{array}{l}17 \\
-- \\
23 \\
17 \\
16 \\
14 \\
17\end{array}$ & $\begin{array}{l}23 \\
-1 \\
29 \\
20 \\
19 \\
12 \\
15\end{array}$ & $\begin{array}{l}25 \\
\ddot{40} \\
31 \\
32 \\
20 \\
20\end{array}$ & $\begin{array}{r}37 \\
30 \\
24 \\
- \\
14 \\
7\end{array}$ & $\begin{array}{l}33 \\
30 \\
28 \\
37 \\
22 \\
35 \\
29\end{array}$ & $\begin{array}{l}37 \\
31 \\
33 \\
-- \\
17 \\
25 \\
21\end{array}$ & $\begin{array}{l}27 \\
-- \\
36 \\
22 \\
19 \\
18 \\
17\end{array}$ & $\begin{array}{l}13 \\
-- \\
25 \\
20 \\
22 \\
17 \\
19\end{array}$ \\
\hline 100 & 0.3 & 13 & 19 & 18 & 24 & 28 & $\cdot 22$ & 19 & 24 & 23 & 16 \\
\hline 110 & $\begin{array}{l}0.3 \\
1.0 \\
2.0 \\
3.0 \\
4.0 \\
5.0 \\
6.0\end{array}$ & $\begin{array}{r}13 \\
15 \\
4 \\
23 \\
-- \\
-- \\
20\end{array}$ & $\begin{array}{r}17 \\
22 \\
25 \\
36 \\
9 \\
-- \\
25\end{array}$ & $\begin{array}{r}16 \\
19 \\
22 \\
31 \\
8 \\
-- \\
22\end{array}$ & $\begin{array}{r}20 \\
24 \\
25 \\
33 \\
8 \\
-- \\
24\end{array}$ & $\begin{array}{l}21 \\
27 \\
28 \\
25 \\
14 \\
-- \\
32\end{array}$ & $\begin{array}{r}21 \\
-2 \\
24 \\
15 \\
15 \\
8 \\
11\end{array}$ & $\begin{array}{l}21 \\
-- \\
32 \\
25 \\
22 \\
17 \\
19\end{array}$ & $\begin{array}{l}25 \\
-- \\
33 \\
22 \\
17 \\
11 \\
15\end{array}$ & $\begin{array}{r}21 \\
26 \\
29 \\
34 \\
8 \\
-- \\
25\end{array}$ & $\begin{array}{r}16 \\
21 \\
23 \\
31 \\
9 \\
-- \\
28\end{array}$ \\
\hline 120 & 0.3 & -- & -- & 4 & 7 & 8 & 19 & 19 & 23 & 7 & 2 \\
\hline 130 & $\begin{array}{l}0.3 \\
1.0 \\
2.0 \\
3.0 \\
4.0 \\
5.0 \\
6.0\end{array}$ & $\begin{array}{l}\because- \\
\therefore- \\
=- \\
=- \\
\because-\end{array}$ & $\begin{array}{l}15 \\
=- \\
=- \\
-- \\
-- \\
--\end{array}$ & $\begin{array}{l}16 \\
-- \\
\square- \\
\square- \\
--\end{array}$ & $\begin{array}{l}19 \\
-- \\
-- \\
-- \\
-- \\
--\end{array}$ & $\begin{array}{l}23 \\
-- \\
-- \\
-- \\
-- \\
--\end{array}$ & $\begin{array}{l}17 \\
22 \\
23 \\
28 \\
4 \\
-2 \\
23\end{array}$ & $\begin{array}{r}20 \\
26 \\
26 \\
35 \\
9 \\
-26\end{array}$ & $\begin{array}{l}20 \\
26 \\
28 \\
30 \\
12 \\
=- \\
26\end{array}$ & $\begin{array}{c}21 \\
-- \\
-- \\
-- \\
-- \\
-- \\
--\end{array}$ & $\begin{array}{l}18 \\
-- \\
-- \\
-- \\
--\end{array}$ \\
\hline 140 & 0.3 & -- & 7 & 9 & 10 & 12 & $\cdots$ & 9 & 8 & 13 & 7 \\
\hline 150 & $\begin{array}{l}0.3 \\
1.0 \\
2.0 \\
3.0 \\
4.0 \\
5.0 \\
6.0\end{array}$ & $\begin{array}{l}=- \\
=- \\
=- \\
=- \\
=- \\
--\end{array}$ & $\begin{array}{r}5 \\
7 \\
18 \\
14 \\
25 \\
16 \\
18\end{array}$ & $\begin{array}{r}8 \\
7 \\
8 \\
15 \\
25 \\
12 \\
14\end{array}$ & $\begin{array}{r}8 \\
8 \\
8 \\
15 \\
27 \\
18 \\
17\end{array}$ & $\begin{array}{l}10 \\
12 \\
13 \\
15 \\
23 \\
21 \\
21\end{array}$ & $\begin{array}{l}16 \\
=- \\
=- \\
=- \\
-- \\
--\end{array}$ & $\begin{array}{l}24 \\
-- \\
-- \\
-- \\
--\end{array}$ & $\begin{array}{l}21 \\
-- \\
-- \\
-- \\
-- \\
--\end{array}$ & $\begin{array}{r}10 \\
9 \\
13 \\
17 \\
29 \\
20 \\
19\end{array}$ & $\begin{array}{r}9 \\
10 \\
12 \\
16 \\
28 \\
20 \\
22\end{array}$ \\
\hline 160 & 0.3 & -- & 8 & 9 & 8 & 8 & 8 & 16 & 15 & 12 & 10 \\
\hline 170 & $\begin{array}{l}0.3 \\
1.0 \\
2.0 \\
3.0 \\
4.0 \\
5.0 \\
6.0\end{array}$ & $\begin{array}{l}-- \\
-- \\
-- \\
-- \\
--\end{array}$ & $\begin{array}{l}-- \\
=- \\
=- \\
=- \\
=- \\
--\end{array}$ & $\begin{array}{l}-- \\
-- \\
-- \\
-- \\
-- \\
--\end{array}$ & $\begin{array}{l}-- \\
-- \\
-- \\
-- \\
-- \\
--\end{array}$ & $\begin{array}{l}-- \\
-- \\
-- \\
-- \\
--\end{array}$ & $\begin{array}{r}4 \\
4 \\
-7 \\
22 \\
17 \\
15\end{array}$ & $\begin{array}{l}16 \\
15 \\
16 \\
18 \\
31 \\
23 \\
22\end{array}$ & $\begin{array}{l}14 \\
12 \\
23 \\
19 \\
31 \\
22 \\
23\end{array}$ & $\begin{array}{l}-- \\
-- \\
-- \\
-- \\
--\end{array}$ & $\begin{array}{l}-- \\
-- \\
-- \\
-- \\
-- \\
--\end{array}$ \\
\hline 180 & 0.3 & -- & 10 & 10 & 8 & 15 & 3 & 13 & 9 & 14 & .11 \\
\hline
\end{tabular}


Table 11

Downwash Velocities, UH-1H Fly-By Tests

Helicopter Gross Weight 9500 lb; Disc Load, 5.25 psf;

Rotor Height Above Ground, $20 \mathrm{ft}$

\begin{tabular}{|c|c|c|c|}
\hline \multirow{2}{*}{$\begin{array}{c}\text { Horizontal } \\
\text { Distance from } \\
\text { Rotor Hub(s), ft } \\
\end{array}$} & \multirow{2}{*}{$\begin{array}{l}\text { Velocity Point } \\
\text { Height Above } \\
\text { Ground, ft } \\
\end{array}$} & \multicolumn{2}{|c|}{$\begin{array}{c}\text { Downwash Velocities, mph, at Indicated } \\
\text { Headings, deg* }\end{array}$} \\
\hline & & $\begin{array}{r}90 \\
\text { deg } \\
\end{array}$ & $\begin{array}{l}270 \\
\text { deg }\end{array}$ \\
\hline 20 & 0.3 & 51 & 27 \\
\hline 30 & $\begin{array}{l}0.3 \\
1.0 \\
2.0 \\
3.0 \\
4.0 \\
5.0 \\
6.0\end{array}$ & $\begin{array}{l}42 \\
42 \\
40 \\
19 \\
-- \\
-- \\
--\end{array}$ & $\begin{array}{r}55 \\
49 \\
55 \\
45 \\
43 \\
20 \\
5\end{array}$ \\
\hline 40 & 0.3 & 30 & 57 \\
\hline 50 & $\begin{array}{l}0.3 \\
1.0 \\
2.0 \\
3.0 \\
4.0 \\
5.0 \\
6.0\end{array}$ & $\begin{array}{r}20 \\
31 \\
16 \\
16 \\
8 \\
8 \\
8\end{array}$ & $\begin{array}{r}38 \\
60 \\
31 \\
32 \\
15 \\
10 \\
5\end{array}$ \\
\hline 70 & $\begin{array}{l}0.3 \\
1.0 \\
2.0 \\
3.0 \\
4.0 \\
5.0 \\
6.0\end{array}$ & $\begin{array}{r}22 \\
13 \\
4 \\
19 \\
12 \\
10 \\
7\end{array}$ & $\begin{array}{l}39 \\
34 \\
30 \\
31 \\
13 \\
16 \\
12\end{array}$ \\
\hline 80 & 0.3 & 12 & 21 \\
\hline 90 & $\begin{array}{l}0.3 \\
1.0 \\
2.0 \\
3.0 \\
4.0 \\
5.0 \\
6.0\end{array}$ & $\begin{array}{l}-- \\
-- \\
-- \\
-- \\
4\end{array}$ & $\begin{array}{r}15 \\
-- \\
28 \\
18 \\
16 \\
9 \\
14\end{array}$ \\
\hline 110 & $\begin{array}{l}0.3 \\
1.0 \\
2.0 \\
3.0 \\
4.0 \\
5.0 \\
6.0\end{array}$ & $\begin{array}{l}-- \\
-- \\
-- \\
-- \\
-- \\
--\end{array}$ & $\begin{array}{l}13 \\
18 \\
37 \\
39 \\
-- \\
-- \\
23\end{array}$ \\
\hline
\end{tabular}

* Heading of helicopter nose relative to line of measurement points. Helicopter at heading of zero degrees faces all measurement points. 
Table 12

Downwash Velocities, UH-IM Lift-Off Tests

Helicopter Gross Weight, 9500 Ib; Disc Load, 5.25 psf;

Rotor Height Above Ground, $14 \mathrm{ft}$

\begin{tabular}{|c|c|c|c|c|c|c|c|c|c|c|c|c|c|}
\hline \multirow{2}{*}{$\begin{array}{c}\text { Horizontal } \\
\text { Distance from } \\
\text { Rotor Hub }(\mathrm{s}) \text {, ft } \\
\end{array}$} & \multirow{2}{*}{$\begin{array}{l}\text { Velocity Point } \\
\text { Height Above } \\
\text { Ground, ft } \\
\end{array}$} & \multicolumn{12}{|c|}{ Downwash Velocities, mph, at Indicated Headings, deg ${ }^{*}$} \\
\hline & & $\begin{array}{c}0 \\
\text { deg }\end{array}$ & $\begin{array}{r}30 \\
\text { deg }\end{array}$ & $\begin{array}{r}60 \\
\text { deg }\end{array}$ & $\begin{array}{l}90 \\
\text { deg }\end{array}$ & $\begin{array}{l}120 \\
\text { deg }\end{array}$ & $\begin{array}{l}150 \\
\text { deg }\end{array}$ & $\begin{array}{l}180 \\
\text { deg }\end{array}$ & $\begin{array}{l}210 \\
\text { deg }\end{array}$ & $\begin{array}{l}240 \\
\text { deg }\end{array}$ & $\begin{array}{l}270 \\
\text { deg }\end{array}$ & $\begin{array}{l}300 \\
\text { deg }\end{array}$ & $\begin{array}{l}330 \\
\text { deg }\end{array}$ \\
\hline 20 & 0.3 & 59 & 53 & 54 & 34 & 69 & 72 & 56 & 75 & 22 & 50 & 56 & 64 \\
\hline 30 & $\begin{array}{l}0.3 \\
1.0 \\
2.0 \\
3.0 \\
4.0 \\
5.0 \\
6.0\end{array}$ & $\begin{array}{l}70 \\
61 \\
66 \\
52 \\
35 \\
18 \\
--\end{array}$ & $\begin{array}{l}68 \\
60 \\
65 \\
47 \\
47 \\
33 \\
--\end{array}$ & $\begin{array}{l}76 \\
64 \\
68 \\
51 \\
32 \\
12 \\
--\end{array}$ & $\begin{array}{l}72 \\
62 \\
71 \\
61 \\
66 \\
22 \\
-\cdots\end{array}$ & $\begin{array}{l}68 \\
59 \\
69 \\
58 \\
50 \\
41 \\
26\end{array}$ & $\begin{array}{l}64 \\
61 \\
60 \\
43 \\
34 \\
28 \\
13\end{array}$ & $\begin{array}{l}56 \\
51 \\
63 \\
62 \\
69 \\
66 \\
47\end{array}$ & $\begin{array}{l}63 \\
62 \\
70 \\
59 \\
59 \\
41 \\
26\end{array}$ & $\begin{array}{l}64 \\
55 \\
64 \\
59 \\
61 \\
61 \\
33\end{array}$ & $\begin{array}{l}63 \\
60 \\
69 \\
62 \\
41 \\
21 \\
2\end{array}$ & $\begin{array}{r}72 \\
61 \\
70 \\
58 \\
50 \\
28 \\
4\end{array}$ & $\begin{array}{l}70 \\
63 \\
72 \\
53 \\
41 \\
15 \\
--\end{array}$ \\
\hline 40 & 0.3 & 64 & 57 & 58 & 51 & 58 & 56 & 49 & 67 & 70 & 72 & 69 & 67 \\
\hline 50 & $\begin{array}{l}0.3 \\
1.0 \\
2.0 \\
3.0 \\
4.0 \\
5.0 \\
6.0\end{array}$ & $\begin{array}{r}37 \\
59 \\
35 \\
37 \\
19 \\
14 \\
6\end{array}$ & $\begin{array}{l}39 \\
33 \\
35 \\
39 \\
24 \\
21 \\
--\end{array}$ & $\begin{array}{r}35 \\
39 \\
36 \\
39 \\
16 \\
14 \\
6\end{array}$ & $\begin{array}{l}42 \\
64 \\
39 \\
41 \\
21 \\
27 \\
30\end{array}$ & $\begin{array}{l}42 \\
66 \\
42 \\
52 \\
35 \\
37 \\
11\end{array}$ & $\begin{array}{l}36 \\
50 \\
31 \\
43 \\
23 \\
28 \\
10\end{array}$ & $\begin{array}{l}29 \\
48 \\
37 \\
52 \\
38 \\
55 \\
36\end{array}$ & $\begin{array}{l}42 \\
66 \\
43 \\
48 \\
23 \\
26 \\
15\end{array}$ & $\begin{array}{l}42 \\
66 \\
43 \\
47 \\
34 \\
29 \\
12\end{array}$ & $\begin{array}{l}43 \\
66 \\
44 \\
45 \\
22 \\
24 \\
16\end{array}$ & $\begin{array}{r}40 \\
66 \\
40 \\
48 \\
23 \\
16 \\
6\end{array}$ & $\begin{array}{r}40 \\
58 \\
35 \\
41 \\
23 \\
18 \\
5\end{array}$ \\
\hline 70 & $\begin{array}{l}0.3 \\
1.0 \\
2.0 \\
3.0 \\
4.0 \\
5.0 \\
6.0\end{array}$ & $\begin{array}{l}41 \\
37 \\
33 \\
38 \\
19 \\
22 \\
10\end{array}$ & $\begin{array}{l}41 \\
34 \\
33 \\
39 \\
19 \\
23 \\
16\end{array}$ & $\begin{array}{l}41 \\
33 \\
30 \\
36 \\
18 \\
14 \\
11\end{array}$ & $\begin{array}{l}44 \\
40 \\
38 \\
40 \\
18 \\
16 \\
13\end{array}$ & $\begin{array}{l}47 \\
45 \\
44 \\
52 \\
24 \\
28 \\
12\end{array}$ & $\begin{array}{l}40 \\
33 \\
32 \\
40 \\
17 \\
18 \\
13\end{array}$ & $\begin{array}{l}40 \\
36 \\
37 \\
44 \\
27 \\
39 \\
28\end{array}$ & $\begin{array}{l}50 \\
43 \\
41 \\
54 \\
21 \\
24 \\
10\end{array}$ & $\begin{array}{r}44 \\
45 \\
52 \\
52 \\
23 \\
4 \\
--\end{array}$ & $\begin{array}{l}50 \\
45 \\
39 \\
44 \\
22 \\
26 \\
11\end{array}$ & $\begin{array}{l}46 \\
40 \\
34 \\
48 \\
22 \\
18 \\
10\end{array}$ & $\begin{array}{l}40 \\
38 \\
33 \\
42 \\
17 \\
17 \\
10\end{array}$ \\
\hline 90 & $\begin{array}{l}0.3 \\
1.0 \\
2.0 \\
3.0 \\
4.0 \\
5.0 \\
6.0\end{array}$ & $\begin{array}{r}24 \\
-- \\
32 \\
24 \\
15 \\
2 \\
12\end{array}$ & $\begin{array}{r}28 \\
-- \\
37 \\
24 \\
19 \\
6 \\
18\end{array}$ & $\begin{array}{r}28 \\
-- \\
37 \\
28 \\
21 \\
3 \\
16\end{array}$ & $\begin{array}{r}32 \\
-- \\
39 \\
27 \\
22 \\
6 \\
17\end{array}$ & $\begin{array}{r}34 \\
38 \\
33 \\
25 \\
6 \\
15\end{array}$ & $\begin{array}{l}28 \\
-- \\
32 \\
21 \\
17 \\
-. \\
13\end{array}$ & $\begin{array}{l}29 \\
41 \\
34 \\
24 \\
13 \\
24\end{array}$ & $\begin{array}{l}35 \\
46 \\
38 \\
28 \\
10 \\
23\end{array}$ & $\begin{array}{r}31 \\
-- \\
42 \\
28 \\
21 \\
5 \\
13\end{array}$ & $\begin{array}{l}32 \\
-- \\
41 \\
23 \\
10 \\
18\end{array}$ & $\begin{array}{l}29 \\
-- \\
39 \\
27 \\
17 \\
-- \\
10\end{array}$ & $\begin{array}{r}29 \\
-- \\
37 \\
26 \\
17 \\
1 \\
14\end{array}$ \\
\hline 100 & 0.3 & 14 & 16 & 18 & 21 & 20 & 13 & $2 I$ & 26 & 21 & 22 & 18 & 28 \\
\hline 110 & $\begin{array}{l}0.3 \\
1.0 \\
2.0 \\
3.0 \\
4.0 \\
5.0 \\
6.0\end{array}$ & $\begin{array}{r}19 \\
26 \\
25 \\
26 \\
9 \\
-16\end{array}$ & $\begin{array}{l}21 \\
27 \\
17 \\
29 \\
15 \\
-- \\
22\end{array}$ & $\begin{array}{l}24 \\
28 \\
25 \\
29 \\
16 \\
-- \\
27\end{array}$ & $\begin{array}{l}26 \\
33 \\
31 \\
34 \\
19 \\
-1 \\
29\end{array}$ & $\begin{array}{l}27 \\
28 \\
31 \\
34 \\
16 \\
-- \\
20\end{array}$ & $\begin{array}{r}23 \\
26 \\
15 \\
25 \\
9 \\
-18\end{array}$ & $\begin{array}{l}23 \\
29 \\
30 \\
37 \\
23 \\
-- \\
30\end{array}$ & $\begin{array}{l}33 \\
35 \\
33 \\
35 \\
19 \\
-27\end{array}$ & $\begin{array}{l}25 \\
31 \\
30 \\
34 \\
19 \\
-- \\
22\end{array}$ & $\begin{array}{l}26 \\
31 \\
30 \\
34 \\
19 \\
-- \\
30\end{array}$ & $\begin{array}{l}22 \\
29 \\
24 \\
29 \\
14 \\
-- \\
19\end{array}$ & $\begin{array}{l}23 \\
27 \\
26 \\
29 \\
10 \\
-18\end{array}$ \\
\hline 120 & 0.3 & 7 & 7 & 12 & 13 & 13 & 8 & 11 & 13 & 11 & 11 & 8 & 8 \\
\hline 130 & 0.3 & 19 & 19 & 18 & 28 & 27 & 23 & 24 & 24 & 21 & 28 & 22 & 21 \\
\hline 140 & 0.3 & 12 & 12 & 16 & 21 & 18 & 15 & 19 & 14 & 18 & 22 & 13 & 14 \\
\hline .1 .50 & $\begin{array}{l}0.3 \\
1.0 \\
2.0 \\
3.0 \\
4.0 \\
5.0 \\
6.0\end{array}$ & $\begin{array}{r}10 \\
10 \\
9 \\
16 \\
22 \\
19 \\
18\end{array}$ & $\begin{array}{r}11 \\
12 \\
8 \\
16 \\
24 \\
21 \\
21\end{array}$ & $\begin{array}{l}16 \\
16 \\
17 \\
18 \\
28 \\
25 \\
25\end{array}$ & $\begin{array}{l}22 \\
17 \\
22 \\
23 \\
30 \\
28 \\
27\end{array}$ & $\begin{array}{l}19 \\
17 \\
18 \\
20 \\
27 \\
28 \\
29\end{array}$ & $\begin{array}{l}12 \\
12 \\
12 \\
16 \\
26 \\
18 \\
18\end{array}$ & $\begin{array}{l}16 \\
19 \\
18 \\
20 \\
30 \\
28 \\
29\end{array}$ & $\begin{array}{l}13 \\
15 \\
15 \\
20 \\
25 \\
22 \\
21\end{array}$ & $\begin{array}{l}16 \\
17 \\
15 \\
18 \\
27 \\
23 \\
21\end{array}$ & $\begin{array}{l}18 \\
15 \\
15 \\
18 \\
26 \\
24 \\
22\end{array}$ & $\begin{array}{l}12 \\
11 \\
12 \\
18 \\
25 \\
24 \\
22\end{array}$ & $\begin{array}{l}12 \\
13 \\
11 \\
16 \\
23 \\
20 \\
21\end{array}$ \\
\hline 160 & 0.3 & 10 & 9 & 12 & 17 & 14 & 10 & 15 & 11 & 21 & 14 & 10 & 12 \\
\hline 170 & 0.3 & 8 & 9 & 11 & 15 & 13 & 8 & 12 & 10 & 10 & 12 & 10 & 11 \\
\hline 180 & 0.3 & 14 & 12 & 17 & 20 & 19 & 12 & 19 & 15 & 16 & 8 & II & 17 \\
\hline 190 & 0.3 & 5 & -- & 7 & 11 & 7 & -- & 10 & 6 & 6 & 7 & 6 & 6 \\
\hline
\end{tabular}

* Heading of hellcopter nose relative to Iine of measurement points. Hellcopter at heading of zero degrees faces all measurement points. 
Table 13

Downwash Velocities, UH-1M Hover Tests

Hellcopter Gross Weight, 9500 lb; Disc Load, 5.25 psf;

Rotor Height Above Ground, 44 and 66 ft

\begin{tabular}{|c|c|c|c|c|c|c|c|c|c|}
\hline \multirow{3}{*}{$\begin{array}{l}\text { Horizontal } \\
\text { Distance from } \\
\text { Rotor Hub }(s), \mathrm{ft} \\
\end{array}$} & \multirow{3}{*}{$\begin{array}{l}\text { Velocity Point } \\
\text { Helght Above } \\
\text { Ground, ft } \\
\end{array}$} & \multirow{2}{*}{\multicolumn{8}{|c|}{$\begin{array}{c}\text { Downwash Velocities, mph, at Indicated Rotor Heights } \\
\text { Above Ground, } \mathrm{ft} \text {, and Headings, deg* } \\
44 \mathrm{ft} \\
\end{array}$}} \\
\hline & & & & & & & & & \\
\hline & & $\begin{array}{c}0 \\
\text { deg }\end{array}$ & $\begin{array}{r}90 \\
\text { deg }\end{array}$ & $\begin{array}{l}180 \\
\text { deg }\end{array}$ & $\begin{array}{l}270 \\
\text { deg }\end{array}$ & $\begin{array}{c}0 \\
\text { deg }\end{array}$ & $\begin{array}{c}90 \\
\text { deg }\end{array}$ & $\begin{array}{l}180 \\
\text { deg }\end{array}$ & $\begin{array}{l}270 \\
\text { deg } \\
\end{array}$ \\
\hline 20 & 0.3 & 45 & 8 & 28 & $15 \ldots$ & 56 & 20 & 37 & 16 \\
\hline 30 & $\begin{array}{l}0.3 \\
1.0 \\
2.0 \\
3.0 \\
4.0 \\
5.0 \\
6.0\end{array}$ & $\begin{array}{l}65 \\
60 \\
64 \\
56 \\
58 \\
61 \\
48\end{array}$ & $\begin{array}{l}75 \\
63 \\
70 \\
66 \\
62 \\
49 \\
33\end{array}$ & $\begin{array}{l}70 \\
32 \\
57 \\
49 \\
54 \\
42 \\
33\end{array}$ & $\begin{array}{l}51 \\
47 \\
57 \\
50 \\
57 \\
53 \\
48\end{array}$ & $\begin{array}{l}67 \\
33 \\
68 \\
53 \\
59 \\
58 \\
48\end{array}$ & $\begin{array}{l}64 \\
48 \\
53 \\
43 \\
48 \\
44 \\
34\end{array}$ & $\begin{array}{l}33 \\
33 \\
28 \\
37 \\
51 \\
50 \\
41\end{array}$ & $\begin{array}{l}70 \\
61 \\
72 \\
61 \\
68 \\
68 \\
43\end{array}$ \\
\hline 40 & 0.3 & 64 & 70 & 65 & 49 & 19 & 64 & 63 & 76 \\
\hline 50 & $\begin{array}{l}0.3 \\
1.0 \\
2.0 \\
3.0 \\
4.0 \\
5.0 \\
6.0\end{array}$ & $\begin{array}{l}44 \\
63 \\
39 \\
55 \\
36 \\
40 \\
23\end{array}$ & $\begin{array}{l}49 \\
74 \\
48 \\
60 \\
32 \\
36 \\
16\end{array}$ & $\begin{array}{l}42 \\
62 \\
40 \\
49 \\
37 \\
46 \\
31\end{array}$ & $\begin{array}{l}48 \\
73 \\
46 \\
56 \\
39 \\
49 \\
30\end{array}$ & $\begin{array}{l}42 \\
60 \\
39 \\
48 \\
28 \\
34 \\
23\end{array}$ & $\begin{array}{l}50 \\
74 \\
45 \\
56 \\
40 \\
61 \\
43\end{array}$ & $\begin{array}{l}42 \\
57 \\
39 \\
54 \\
37 \\
58 \\
46\end{array}$ & $\begin{array}{l}49 \\
75 \\
50 \\
55 \\
35 \\
40 \\
21\end{array}$ \\
\hline 70 & $\begin{array}{l}0.3 \\
1.0 \\
2.0 \\
3.0 \\
4.0 \\
5.0 \\
6.0\end{array}$ & $\begin{array}{l}43 \\
40 \\
38 \\
46 \\
24 \\
27 \\
18\end{array}$ & $\begin{array}{l}53 \\
49 \\
46 \\
49 \\
25 \\
35 \\
13\end{array}$ & $\begin{array}{l}51 \\
47 \\
38 \\
42 \\
20 \\
22 \\
16\end{array}$ & $\begin{array}{l}53 \\
44 \\
42 \\
52 \\
24 \\
36 \\
22\end{array}$ & $\begin{array}{l}50 \\
40 \\
46 \\
50 \\
23 \\
26 \\
24\end{array}$ & $\begin{array}{l}60 \\
53 \\
45 \\
52 \\
33 \\
44 \\
32\end{array}$ & $\begin{array}{l}62 \\
50 \\
43 \\
53 \\
30 \\
45 \\
34\end{array}$ & $\begin{array}{l}54 \\
53 \\
44 \\
55 \\
27 \\
34 \\
19\end{array}$ \\
\hline 90 & $\begin{array}{l}0.3 \\
1.0 \\
2.0 \\
3.0 \\
4.0 \\
5.0 \\
6.0\end{array}$ & $\begin{array}{l}29 \\
-- \\
42 \\
32 \\
23 \\
11 \\
23\end{array}$ & $\begin{array}{l}37 \\
-- \\
48 \\
33 \\
25 \\
12 \\
20\end{array}$ & $\begin{array}{r}36 \\
-- \\
46 \\
25 \\
17 \\
2 \\
16\end{array}$ & $\begin{array}{l}33 \\
-- \\
36 \\
34 \\
25 \\
22 \\
25\end{array}$ & $\begin{array}{r}32 \\
-- \\
42 \\
35 \\
24 \\
9 \\
21\end{array}$ & $\begin{array}{l}37 \\
-19 \\
41 \\
31 \\
20 \\
25\end{array}$ & $\begin{array}{l}46 \\
-2 \\
56 \\
42 \\
29 \\
18 \\
30\end{array}$ & $\begin{array}{l}40 \\
-- \\
50 \\
35 \\
24 \\
10 \\
18\end{array}$ \\
\hline 100 & 0.3 & 23 & 27 & 24 & 20 & 24 & 27 & 38 & 27 \\
\hline 110 & $\begin{array}{l}0.3 \\
1.0 \\
2.0 \\
3.0 \\
4.0 \\
5.0 \\
6.0\end{array}$ & $\begin{array}{l}23 \\
33 \\
34 \\
35 \\
19 \\
-- \\
37\end{array}$ & $\begin{array}{l}30 \\
38 \\
38 \\
40 \\
19 \\
-- \\
26\end{array}$ & $\begin{array}{l}30 \\
31 \\
27 \\
34 \\
12 \\
-- \\
20\end{array}$ & $\begin{array}{l}27 \\
35 \\
35 \\
42 \\
22 \\
-- \\
29\end{array}$ & $\begin{array}{l}28 \\
34 \\
31 \\
32 \\
19 \\
-26\end{array}$ & $\begin{array}{l}28 \\
38 \\
37 \\
44 \\
30 \\
-- \\
31\end{array}$ & $\begin{array}{l}38 \\
48 \\
47 \\
48 \\
28 \\
-- \\
37\end{array}$ & $\begin{array}{l}33 \\
37 \\
36 \\
39 \\
27 \\
-16\end{array}$ \\
\hline 120 & 0.3 & 10 & 14 & 13 & 13 & 13 & 14 & 20 & 18 \\
\hline 130 & 0.3 & 25 & 28 & 26 & 27 & 26 & 29 & 30 & 28 \\
\hline 140 & 0.3 & 19 & 22 & 19 & 19 & 16 & 22 & 25 & 21 \\
\hline 150 & $\begin{array}{l}0.3 \\
1.0 \\
2.0 \\
3.0 \\
4.0 \\
5.0 \\
6.0\end{array}$ & $\begin{array}{l}16 \\
19 \\
22 \\
22 \\
30 \\
29 \\
30\end{array}$ & $\begin{array}{l}19 \\
21 \\
23 \\
21 \\
30 \\
28 \\
28\end{array}$ & $\begin{array}{l}17 \\
17 \\
17 \\
19 \\
27 \\
20 \\
20\end{array}$ & $\begin{array}{l}18 \\
18 \\
19 \\
23 \\
32 \\
31 \\
30\end{array}$ & $\begin{array}{l}10 \\
16 \\
18 \\
20 \\
27 \\
26 \\
26\end{array}$ & $\begin{array}{l}25 \\
27 \\
26 \\
25 \\
34 \\
30 \\
32\end{array}$ & $\begin{array}{l}23 \\
20 \\
29 \\
25 \\
34 \\
35 \\
35\end{array}$ & $\begin{array}{l}18 \\
19 \\
13 \\
17 \\
26 \\
28 \\
28\end{array}$ \\
\hline 160 & 0.3 & 16 & 15 & 13 & 26 & 14 & 19 & 27 & 10 \\
\hline 170 & 0.3 & 16 & 13 & 13 & 15 & 12 & 16 & 15 & 15 \\
\hline 180 & 0.3 & 21 & 19 & 19 & 20 & 18 & 20 & 23 & 20 \\
\hline 190 & 0.3 & 11 & 10 & 10 & 12 & 8 & 10 & 13 & 10 \\
\hline
\end{tabular}

* Heading of hellcopter nose relative to line of measurement points. Helicopter at heading of zero degrees faces all measurement points. 
Table 14

Downwash Velocities, CH-47 Lift-Off Tests

Helicopter Gross Weight, 36,000 lb; Disc Load, 6.37 psf;

Rotor Height Above Ground, $15 \mathrm{ft}$

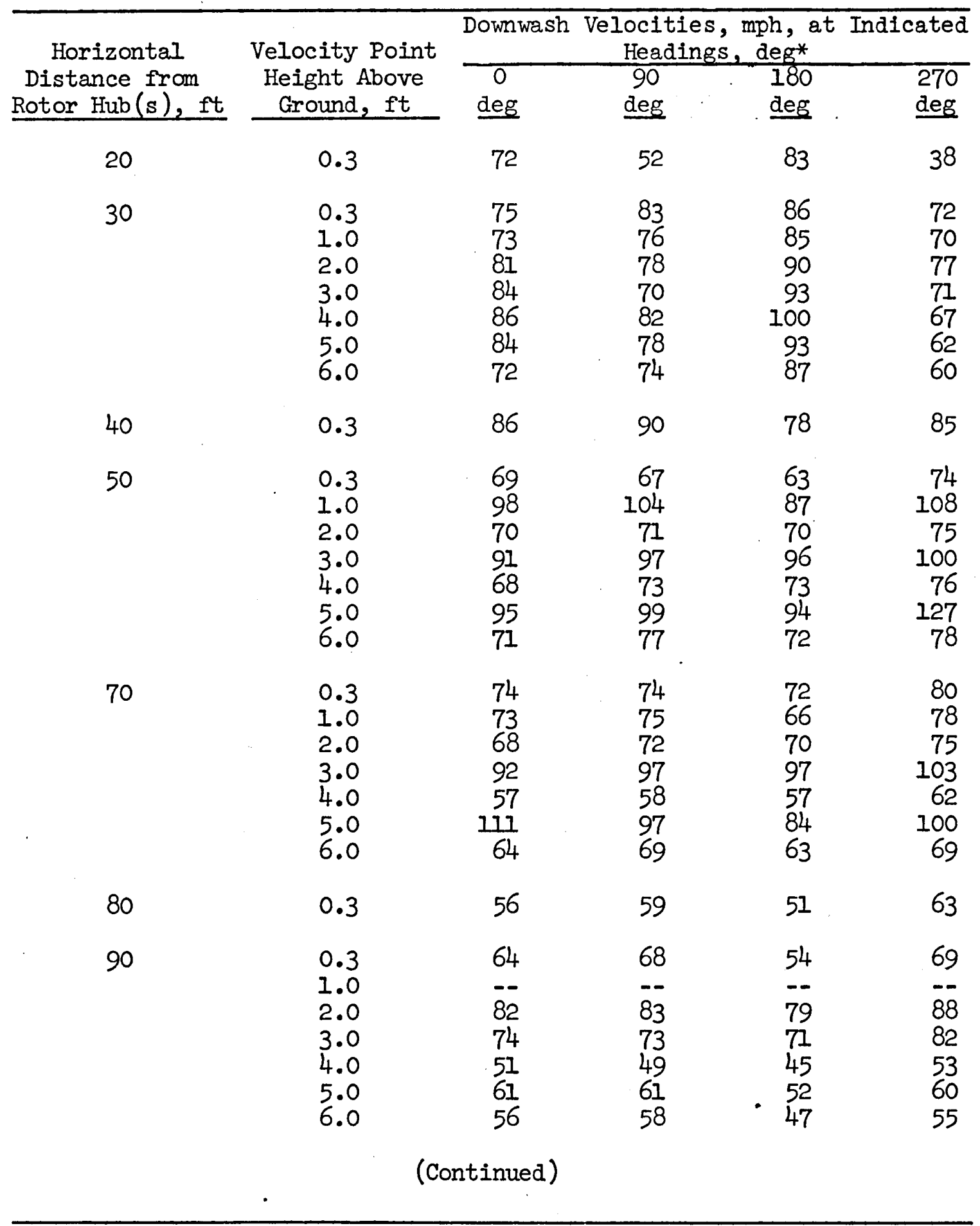

* Heading of helicopter nose relative to line of measurement points. Helicopter at heading of zero degrees faces all measurement points. 
Table 14 (Concluded)

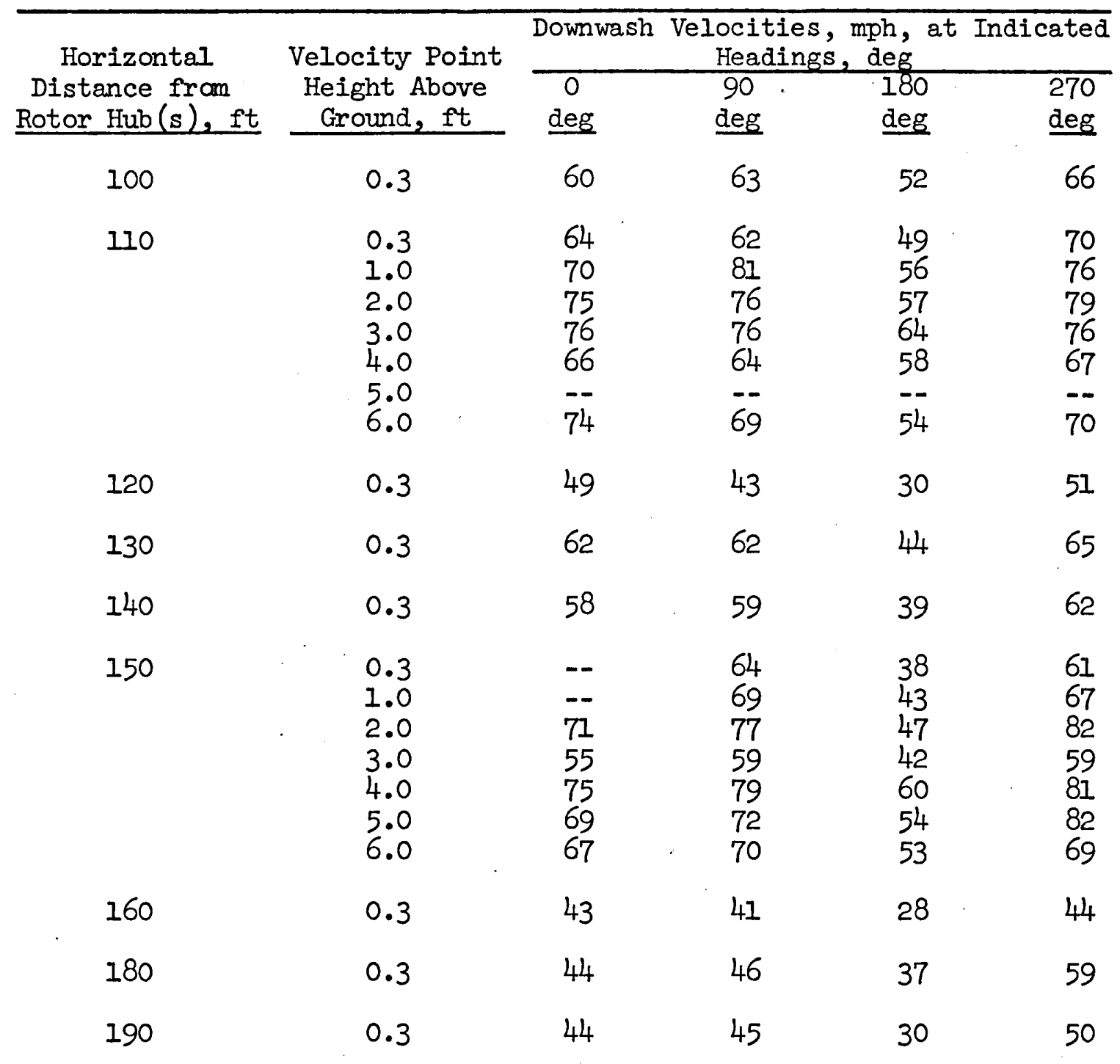


Table 15

Downwash Velocitles, $\mathrm{CH}-47$ Hover Tests

Helicopter Gross Weight, 36,000 Ib; Disc Load, 6.37 psf;

Rotor Helght Above Ground, 50 and $90 \mathrm{ft}$..

\begin{tabular}{|c|c|c|c|c|c|c|c|c|c|c|c|c|c|}
\hline \multirow[b]{2}{*}{$\begin{array}{c}\text { Horizontal } \\
\text { Distance fram } \\
\text { Rotor Hub (s), ftt }\end{array}$} & \multirow[b]{2}{*}{$\begin{array}{l}\text { Velocity Point } \\
\text { Height Above } \\
\text { Ground, ft }\end{array}$} & \multicolumn{12}{|c|}{$\begin{array}{l}\text { Downwash Velocities, mph, at Indicated Rotor Heights } \\
\text { Above Ground, ft, and Headings, deg* }\end{array}$} \\
\hline & & $\begin{array}{l}0 \\
\text { deg }\end{array}$ & $\begin{array}{l}30 \\
\text { deg }\end{array}$ & $\begin{array}{l}60 \\
\text { deg } \\
\end{array}$ & $\begin{array}{l}90 \\
\text { deg } \\
\end{array}$ & $\begin{array}{l}120 \\
\text { deg }\end{array}$ & $\begin{array}{l}150 \\
\text { deg }\end{array}$ & $\begin{array}{l}180 \\
\text { deg }\end{array}$ & $\begin{array}{l}210 \\
\text { deg }\end{array}$ & $\begin{array}{l}240 \\
\text { deg } \\
\end{array}$ & $\begin{array}{l}270 \\
\text { deg } \\
\end{array}$ & $\begin{array}{l}300 \\
\text { deg } \\
\end{array}$ & $\begin{array}{l}330 \\
\text { deg }\end{array}$ \\
\hline 20 & 0.3 & 82 & -- & 29 & 21 & 73 & 94 & 78 & 98 & 32 & 40 & 57 & 81 \\
\hline 30 & $\begin{array}{l}0.3 \\
1.0 \\
2.0 \\
3.0 \\
4.0 \\
5.0 \\
6.0\end{array}$ & $\begin{array}{l}80 \\
79 \\
78 \\
65 \\
67 \\
62 \\
53\end{array}$ & $\begin{array}{l}34 \\
49 \\
35 \\
15 \\
15 \\
13 \\
--\end{array}$ & $\begin{array}{l}28 \\
30 \\
39 \\
21 \\
11 \\
10 \\
14\end{array}$ & $\begin{array}{l}47 \\
44 \\
48 \\
43 \\
48 \\
48 \\
51\end{array}$ & $\begin{array}{l}90 \\
79 \\
83 \\
74 \\
70 \\
69 \\
77\end{array}$ & $\begin{array}{l}84 \\
84 \\
97 \\
92 \\
91 \\
74 \\
63\end{array}$ & $\begin{array}{l}77 \\
75 \\
77 \\
74 \\
83 \\
83 \\
78\end{array}$ & $\begin{array}{r}93 \\
94 \\
98 \\
103 \\
102 \\
99 \\
96\end{array}$ & $\begin{array}{l}72 \\
67 \\
75 \\
71 \\
72 \\
66 \\
54\end{array}$ & $\begin{array}{l}63 \\
56 \\
64 \\
45 \\
56 \\
54 \\
45\end{array}$ & $\begin{array}{l}53 \\
55 \\
64 \\
65 \\
69 \\
64 \\
44\end{array}$ & $\begin{array}{l}68 \\
72 \\
80 \\
76 \\
70 \\
62 \\
43\end{array}$ \\
\hline 40 & 0.3 & 75 & 58 & 57 & 73 & 89 & 79 & 80 & 90 & 102 & 82 & 53 & 58 \\
\hline 50 & $\begin{array}{l}0.3 \\
1.0 \\
2.0 \\
3.0 \\
4.0 \\
5.0 \\
6.0\end{array}$ & $\begin{array}{l}54 \\
80 \\
59 \\
84 \\
57 \\
78 \\
60\end{array}$ & $\begin{array}{l}54 \\
71 \\
38 \\
50 \\
36 \\
55 \\
44\end{array}$ & $\begin{array}{l}59 \\
78 \\
51 \\
60 \\
45 \\
68 \\
54\end{array}$ & $\begin{array}{l}64 \\
94 \\
65 \\
89 \\
68 \\
94 \\
75\end{array}$ & $\begin{array}{l}64 \\
92 \\
63 \\
84 \\
64 \\
88 \\
66\end{array}$ & $\begin{array}{l}55 \\
81 \\
82 \\
88 \\
61 \\
87 \\
66\end{array}$ & $\begin{array}{l}54 \\
96 \\
62 \\
87 \\
57 \\
87 \\
67\end{array}$ & $\begin{array}{r}56 \\
101 \\
74 \\
98 \\
68 \\
91 \\
64\end{array}$ & $\begin{array}{r}78 \\
112 \\
73 \\
90 \\
67 \\
92 \\
74\end{array}$ & $\begin{array}{l}61 \\
89 \\
61 \\
80 \\
53 \\
72 \\
66\end{array}$ & $\begin{array}{l}56 \\
85 \\
55 \\
73 \\
55 \\
73 \\
55\end{array}$ & $\begin{array}{l}41 \\
68 \\
51 \\
66 \\
44 \\
61 \\
45\end{array}$ \\
\hline 70 & $\begin{array}{l}0.3 \\
1.0 \\
2.0 \\
3.0 \\
4.0 \\
5.0 \\
6.0\end{array}$ & $\begin{array}{l}62 \\
57 \\
58 \\
80 \\
50 \\
75 \\
53\end{array}$ & $\begin{array}{l}72 \\
76 \\
67 \\
86 \\
57 \\
90 \\
64\end{array}$ & $\begin{array}{l}73 \\
64 \\
64 \\
90 \\
56 \\
94 \\
61\end{array}$ & $\begin{array}{l}79 \\
73 \\
69 \\
97 \\
60 \\
94 \\
70\end{array}$ & $\begin{array}{l}62 \\
59 \\
56 \\
75 \\
81 \\
67 \\
48\end{array}$ & $\begin{array}{l}62 \\
52 \\
49 \\
67 \\
47 \\
75 \\
61\end{array}$ & $\begin{array}{l}55 \\
53 \\
58 \\
77 \\
48 \\
79 \\
57\end{array}$ & $\begin{array}{l}66 \\
66 \\
75 \\
94 \\
58 \\
89 \\
67\end{array}$ & $\begin{array}{r}7- \\
82 \\
74 \\
104 \\
74 \\
94 \\
59\end{array}$ & $\begin{array}{l}78 \\
73 \\
70 \\
92 \\
54 \\
83 \\
58\end{array}$ & $\begin{array}{l}62 \\
60 \\
59 \\
85 \\
53 \\
82 \\
58\end{array}$ & $\begin{array}{l}49 \\
46 \\
45 \\
60 \\
37 \\
52 \\
38\end{array}$ \\
\hline 80 & 0.3 & 51 & 60 & 58 & 63 & 52 & 43 & 48 & 50 & - & 59 & 51 & 30 \\
\hline 90 & $\begin{array}{l}0.3 \\
1.0 \\
2.0 \\
3.0 \\
4.0 \\
5.0 \\
6.0\end{array}$ & $\begin{array}{l}51 \\
-12 \\
72 \\
62 \\
40 \\
40 \\
29\end{array}$ & $\begin{array}{l}70 \\
-- \\
60 \\
69 \\
46 \\
50 \\
46\end{array}$ & $\begin{array}{l}69 \\
-- \\
82 \\
68 \\
47 \\
54 \\
43\end{array}$ & $\begin{array}{l}73 \\
-8 \\
89 \\
78 \\
57 \\
66 \\
62\end{array}$ & $\begin{array}{l}58 \\
-- \\
77 \\
66 \\
50 \\
57 \\
50\end{array}$ & $\begin{array}{l}45 \\
- \\
59 \\
52 \\
40 \\
47 \\
45\end{array}$ & $\begin{array}{l}46 \\
-00 \\
54 \\
41 \\
47 \\
45\end{array}$ & $\begin{array}{l}65 \\
-- \\
77 \\
68 \\
51 \\
55 \\
50\end{array}$ & $\begin{array}{l}77 \\
-- \\
90 \\
77 \\
54 \\
58 \\
62\end{array}$ & $\begin{array}{l}64 \\
-- \\
82 \\
70 \\
46 \\
49 \\
43\end{array}$ & $\begin{array}{l}62 \\
-- \\
74 \\
65 \\
45 \\
53 \\
49\end{array}$ & $\begin{array}{l}35 \\
-- \\
52 \\
43 \\
30 \\
35 \\
33\end{array}$ \\
\hline 100 & $\begin{array}{l}0.3 \\
1.0 \\
2.0 \\
3.0 \\
4.0 \\
5.0 \\
6.0\end{array}$ & $\begin{array}{l}48 \\
54 \\
38 \\
56 \\
48 \\
-- \\
52\end{array}$ & $\begin{array}{l}61 \\
68 \\
72 \\
74 \\
56 \\
-- \\
45\end{array}$ & $\begin{array}{l}62 \\
71 \\
74 \\
73 \\
59 \\
-56\end{array}$ & $\begin{array}{l}68 \\
81 \\
80 \\
79 \\
69 \\
-- \\
75\end{array}$ & $\begin{array}{l}49 \\
61 \\
52 \\
82 \\
51 \\
-76\end{array}$ & $\begin{array}{l}40 \\
49 \\
44 \\
49 \\
40 \\
- \\
50\end{array}$ & $\begin{array}{l}46 \\
52 \\
48 \\
51 \\
39 \\
-- \\
55\end{array}$ & $\begin{array}{l}54 \\
65 \\
59 \\
66 \\
58 \\
-- \\
60\end{array}$ & $\begin{array}{l}64 \\
70 \\
74 \\
77 \\
63 \\
--60\end{array}$ & $\begin{array}{l}63 \\
68 \\
69 \\
74 \\
63 \\
-- \\
57\end{array}$ & $\begin{array}{l}56 \\
65 \\
60 \\
68 \\
60 \\
-- \\
55\end{array}$ & $\begin{array}{l}29 \\
45 \\
42 \\
44 \\
28 \\
-1\end{array}$ \\
\hline 120 & 0.3 & 29 & 44 & 47 & 47 & 30 & 25 & 23 & 34 & 43 & 40 & 35 & 20 \\
\hline 130 & 0.3 & 43 & 54 & 65 & 66 & 46 & 41 & 41 & 54 & 62 & 50 & 51 & 32 \\
\hline 140 & 0.3 & 34 & 48 & 47 & 62 & 36 & 38 & 36 & 42 & 60 & 45 & 48 & 21 \\
\hline .150 & $\begin{array}{l}0.3 \\
1.0 \\
2.0 \\
3.0 \\
4.0 \\
5.0 \\
6.0\end{array}$ & $\begin{array}{l}38 \\
36 \\
36 \\
32 \\
47 \\
43 \\
45\end{array}$ & $\begin{array}{l}-52 \\
55 \\
59 \\
45 \\
64 \\
55 \\
52\end{array}$ & $\begin{array}{l}56 \\
67 \\
70 \\
53 \\
70 \\
61 \\
62\end{array}$ & $\begin{array}{l}66 \\
82 \\
85 \\
62 \\
85 \\
76 \\
78\end{array}$ & $\begin{array}{l}36 \\
36 \\
48 \\
42 \\
56 \\
50 \\
49\end{array}$ & $\begin{array}{l}37 \\
41 \\
46 \\
37 \\
57 \\
53 \\
52\end{array}$ & $\begin{array}{l}38 \\
45 \\
48 \\
36 \\
49 \\
44 \\
46\end{array}$ & $\begin{array}{l}89 \\
50 \\
54 \\
46 \\
64 \\
57 \\
60\end{array}$ & $\begin{array}{l}57 \\
65 \\
63 \\
39 \\
68 \\
62 \\
63\end{array}$ & $\begin{array}{l}41 \\
45 \\
53 \\
45 \\
65 \\
53 \\
56\end{array}$ & $\begin{array}{l}46 \\
52 \\
58 \\
46 \\
64 \\
56 \\
55\end{array}$ & $\begin{array}{l}23 \\
24 \\
25 \\
24 \\
35 \\
28 \\
28\end{array}$ \\
\hline 160 & 0.3 & 26 & 35 & 41 & 45 & 25 & 27 & 29 & 34 & 37 & 30 & 31 & 20 \\
\hline 180 & 0.3 & 30 & 45 & 46 & 57 & 33 & 31 & 36 & 40 & 44 & 38 & 34 & 27 \\
\hline 190 & 0.3 & 23 & 37 & 42 & 49 & 21 & 25 & 24 & 34 & 40 & 26 & 23 & 10 \\
\hline
\end{tabular}

* Heading of hellcopter nose relative to line of measurement points. Helicopter at heading of zero degrees faces all measurement points. 
Table 15 (Concluded)

\begin{tabular}{|c|c|c|c|c|c|c|c|c|c|c|c|c|c|}
\hline \multirow{3}{*}{$\begin{array}{c}\text { Horizontal } \\
\text { Distance fram } \\
\text { Rotor Hub }(\mathrm{s}), \mathrm{ft} \\
\end{array}$} & \multirow{3}{*}{$\begin{array}{l}\text { Velocity Point } \\
\text { Helght Above } \\
\text { Ground, ft }\end{array}$} & \multicolumn{12}{|c|}{$\begin{array}{l}\text { Downwash Velocities, mph, at Indicated Rotor Heights } \\
\text { Above Ground, ft, and Headings, deg }\end{array}$} \\
\hline & & $\overline{0}$ & 30 & 60 & 90 & 120 & 150 & 180 & 210 & 240 & 270 & 300 & 330 \\
\hline & & deg & deg & deg & deg & deg & deg & deg & deg & deg & deg & deg & deg \\
\hline 20 & 0.3 & 76 & 77 & 32 & 35 & 66 & 65 & 107 & 95 & 81 & 26 & 41 & 70 \\
\hline 30 & $\begin{array}{l}0.3 \\
1.0 \\
2.0 \\
3.0 \\
4.0 \\
5.0 \\
6.0\end{array}$ & $\begin{array}{l}62 \\
66 \\
86 \\
85 \\
86 \\
80 \\
76\end{array}$ & $\begin{array}{l}80 \\
74 \\
82 \\
77 \\
82 \\
78 \\
61\end{array}$ & $\begin{array}{l}47 \\
45 \\
42 \\
38 \\
43 \\
44 \\
43\end{array}$ & $\begin{array}{l}42 \\
32 \\
35 \\
21 \\
26 \\
19 \\
28\end{array}$ & $\begin{array}{l}72 \\
60 \\
57 \\
45 \\
42 \\
33 \\
25\end{array}$ & $\begin{array}{l}87 \\
76 \\
72 \\
53 \\
53 \\
40 \\
39\end{array}$ & $\begin{array}{l}61 \\
81 \\
85 \\
76 \\
68 \\
60 \\
62\end{array}$ & $\begin{array}{l}92 \\
82 \\
70 \\
63 \\
61 \\
55 \\
57\end{array}$ & $\begin{array}{r}111 \\
89 \\
91 \\
76 \\
83 \\
80 \\
69\end{array}$ & $\begin{array}{l}61 \\
53 \\
51 \\
46 \\
54 \\
51 \\
46\end{array}$ & $\begin{array}{l}30 \\
34 \\
37 \\
36 \\
49 \\
52 \\
65\end{array}$ & $\begin{array}{l}44 \\
54 \\
56 \\
49 \\
49 \\
48 \\
46\end{array}$ \\
\hline 40 & 0.3 & 62 & 80 & 72 & 68 & 76 & 92 & 90 & 87 & 104 & 87 & 57 & 62 \\
\hline 50 & $\begin{array}{l}0.3 \\
1.0 \\
2.0 \\
3.0 \\
4.0 \\
5.0 \\
6.0\end{array}$ & $\begin{array}{l}46 \\
64 \\
57 \\
81 \\
55 \\
83 \\
61\end{array}$ & $\begin{array}{l}58 \\
84 \\
63 \\
82 \\
57 \\
76 \\
56\end{array}$ & $\begin{array}{l}64 \\
92 \\
69 \\
90 \\
86 \\
98 \\
79\end{array}$ & $\begin{array}{l}65 \\
92 \\
64 \\
88 \\
70 \\
94 \\
76\end{array}$ & $\begin{array}{l}61 \\
88 \\
60 \\
75 \\
55 \\
75 \\
60\end{array}$ & $\begin{array}{l}65 \\
96 \\
59 \\
77 \\
61 \\
72 \\
54\end{array}$ & $\begin{array}{l}63 \\
92 \\
61 \\
58 \\
51 \\
78 \\
55\end{array}$ & $\begin{array}{l}61 \\
89 \\
57 \\
82 \\
54 \\
81 \\
58\end{array}$ & $\begin{array}{r}73 \\
104 \\
70 \\
90 \\
62 \\
86 \\
63\end{array}$ & $\begin{array}{r}67 \\
100 \\
69 \\
91 \\
69 \\
97 \\
74\end{array}$ & $\begin{array}{l}45 \\
68 \\
48 \\
61 \\
34 \\
60 \\
47\end{array}$ & $\begin{array}{l}45 \\
71 \\
53 \\
69 \\
39 \\
64 \\
50\end{array}$ \\
\hline 70 & $\begin{array}{l}0.3 \\
1.0 \\
2.0 \\
3.0 \\
4.0 \\
5.0 \\
6.0\end{array}$ & $\begin{array}{l}57 \\
58 \\
56 \\
79 \\
47 \\
75 \\
58\end{array}$ & $\begin{array}{l}64 \\
62 \\
54 \\
71 \\
43 \\
60 \\
43\end{array}$ & $\begin{array}{r}79 \\
77 \\
72 \\
102 \\
66 \\
104 \\
74\end{array}$ & $\begin{array}{r}84 \\
86 \\
76 \\
107 \\
67 \\
107 \\
75\end{array}$ & $\begin{array}{l}64 \\
-5 \\
58 \\
78 \\
45 \\
68 \\
46\end{array}$ & $\begin{array}{l}76 \\
75 \\
71 \\
96 \\
58 \\
92 \\
64\end{array}$ & $\begin{array}{l}69 \\
69 \\
66 \\
92 \\
55 \\
86 \\
64\end{array}$ & $\begin{array}{l}61 \\
60 \\
64 \\
82 \\
47 \\
77 \\
58\end{array}$ & $\begin{array}{l}85 \\
79 \\
72 \\
90 \\
55 \\
92 \\
60\end{array}$ & $\begin{array}{l}87 \\
77 \\
71 \\
96 \\
58 \\
93 \\
66\end{array}$ & $\begin{array}{r}70 \\
67 \\
72 \\
104 \\
66 \\
81 \\
58\end{array}$ & $\begin{array}{l}71 \\
65 \\
60 \\
83 \\
80 \\
78 \\
60\end{array}$ \\
\hline 80 & 0.3 & 47 & 43 & 61 & 64 & 49 & 59 & 55 & 49 & 65 & 64 & 55 & 56 \\
\hline 90 & $\begin{array}{l}0.3 \\
1.0 \\
2.0 \\
3.0 \\
4.0 \\
5.0 \\
6.0\end{array}$ & $\begin{array}{l}47 \\
-- \\
56 \\
39 \\
45 \\
43\end{array}$ & $\begin{array}{l}50 \\
-- \\
59 \\
50 \\
34 \\
38 \\
36\end{array}$ & $\begin{array}{l}72 \\
\overline{94} \\
84 \\
60 \\
71 \\
64\end{array}$ & $\begin{array}{l}72 \\
-- \\
91 \\
82 \\
58 \\
68 \\
64\end{array}$ & $\begin{array}{l}49 \\
-- \\
68 \\
56 \\
40 \\
39 \\
39\end{array}$ & $\begin{array}{l}70 \\
-- \\
86 \\
77 \\
53 \\
63 \\
58\end{array}$ & $\begin{array}{l}65 \\
-- \\
81 \\
72 \\
52 \\
-- \\
56\end{array}$ & $\begin{array}{l}57 \\
-- \\
68 \\
62 \\
42 \\
48 \\
46\end{array}$ & $\begin{array}{l}73 \\
--87 \\
72 \\
44 \\
56 \\
51\end{array}$ & $\begin{array}{l}71 \\
-86 \\
76 \\
49 \\
56 \\
53\end{array}$ & $\begin{array}{l}65 \\
-- \\
79 \\
62 \\
38 \\
42 \\
37\end{array}$ & $\begin{array}{l}49 \\
-- \\
72 \\
62 \\
44 \\
45 \\
45\end{array}$ \\
\hline 100 & 0.3 & 44 & 48 & 68 & 66 & 48 & 63 & 60 & 52 & 67 & 67 & 63 & 47 \\
\hline 110 & $\begin{array}{l}0.3 \\
1.0 \\
2.0 \\
3.0 \\
4.0 \\
5.0\end{array}$ & $\begin{array}{l}40 \\
57 \\
54 \\
59 \\
49 \\
--\end{array}$ & $\begin{array}{l}40 \\
49 \\
50 \\
55 \\
41 \\
--\end{array}$ & $\begin{array}{l}64 \\
76 \\
91 \\
83 \\
77 \\
--\end{array}$ & $\begin{array}{l}85 \\
82 \\
83 \\
72 \\
-\end{array}$ & $\begin{array}{l}43 \\
60 \\
51 \\
52 \\
38 \\
--\end{array}$ & $\begin{array}{l}58 \\
72 \\
71 \\
76 \\
69 \\
-\end{array}$ & $\begin{array}{l}52 \\
68 \\
70 \\
73 \\
63 \\
--\end{array}$ & $\begin{array}{l}48 \\
58 \\
57 \\
59 \\
45 \\
--\end{array}$ & $\begin{array}{l}64 \\
70 \\
78 \\
78 \\
67 \\
--\end{array}$ & $\begin{array}{l}68 \\
77 \\
77 \\
79 \\
65 \\
-\end{array}$ & $\begin{array}{l}-- \\
70 \\
69 \\
66 \\
49 \\
--\end{array}$ & $\begin{array}{l}45 \\
52 \\
50 \\
57 \\
45 \\
--\end{array}$ \\
\hline & 6. & 54 & 46 & 79 & 79 & 42 & 76 & 69 & 49 & 68 & 59 & 48 & 61 \\
\hline 120 & 0.3 & 28 & 26 & 49 & 48 & 31 & 42 & 40 & 35 & 47 & 51 & 37 & 25 \\
\hline 130 & 0.3 & 44 & 42 & 71 & 70 & 41 & 59 & 54 & 49 & 62 & 67 & 48 & 38 \\
\hline 140 & 0.3 & 46 & 36 & 67 & 66 & 38 & 56 & 48 & 40 & 57 & 61 & 44 & 42 \\
\hline 150 & $\begin{array}{l}0.3 \\
1.0 \\
2.0 \\
3.0 \\
4.0 \\
5.0 \\
6.0\end{array}$ & $\begin{array}{l}37 \\
40 \\
44 \\
35 \\
51 \\
47 \\
49\end{array}$ & $\begin{array}{l}30 \\
37 \\
44 \\
39 \\
53 \\
44 \\
42\end{array}$ & $\begin{array}{l}7- \\
75 \\
83 \\
46 \\
84 \\
79 \\
80\end{array}$ & $\begin{array}{l}66 \\
81 \\
83 \\
64 \\
91 \\
84 \\
83\end{array}$ & $\begin{array}{l}34 \\
38 \\
41 \\
36 \\
51 \\
46 \\
45\end{array}$ & $\begin{array}{l}58 \\
68 \\
75 \\
59 \\
82 \\
72 \\
72\end{array}$ & $\begin{array}{l}48 \\
56 \\
63 \\
52 \\
72 \\
68 \\
71\end{array}$ & $\begin{array}{l}36 \\
40 \\
41 \\
36 \\
55 \\
48 \\
51\end{array}$ & $\begin{array}{l}58 \\
63 \\
76 \\
58 \\
87 \\
69 \\
65\end{array}$ & $\begin{array}{l}63 \\
65 \\
70 \\
53 \\
71 \\
63 \\
61\end{array}$ & $\begin{array}{l}42 \\
49 \\
54 \\
43 \\
63 \\
55 \\
52\end{array}$ & $\begin{array}{l}30 \\
33 \\
37 \\
34 \\
41 \\
41 \\
41\end{array}$ \\
\hline 160 & 0.3 & 24 & 23 & 44 & 4 & 26 & 40 & 30 & 23 & 39 & 45 & 30 & 22 \\
\hline .180 & 0.3 & 23 & 18 & 57 & 61 & 33 & 52 & 43 & 29 & 51 & 54 & 34 & 19 \\
\hline 190 & 0.3 & 17 & 10 & 53 & 56 & 27 & 45 & 34 & 23 & 46 & 48 & 32 & 21 \\
\hline
\end{tabular}




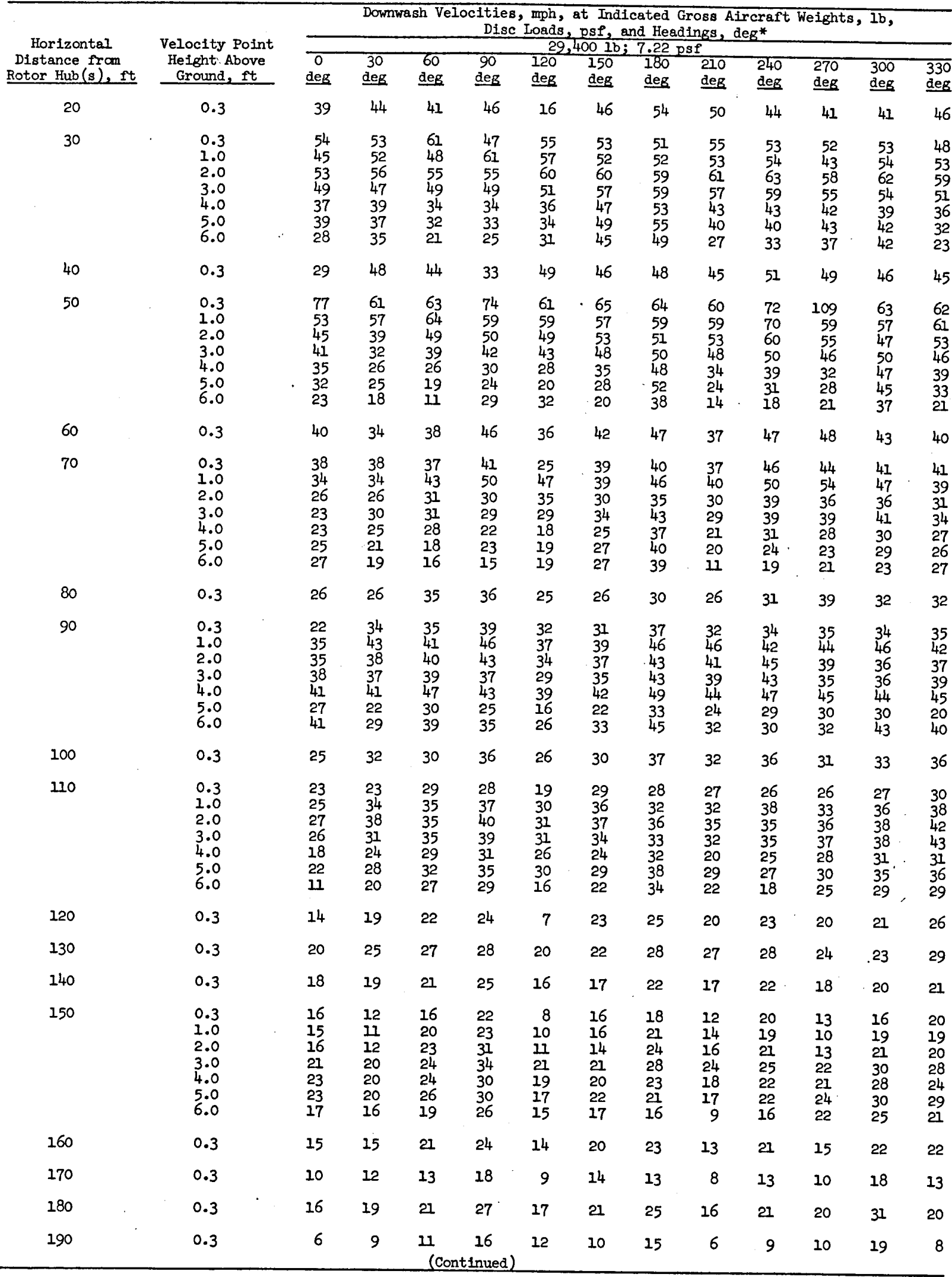




\begin{tabular}{|c|c|c|c|c|c|c|c|c|c|c|c|c|c|c|}
\hline \multirow{3}{*}{$\begin{array}{c}\text { Horizontal } \\
\text { Distance fram } \\
\text { Rotor Hub }(\mathrm{s}), \mathrm{ft}\end{array}$} & \multirow{3}{*}{$\begin{array}{l}\text { Velocity Point } \\
\text { Helght Above } \\
\text { Ground, ft } \\
\end{array}$} & \multicolumn{13}{|c|}{$\begin{array}{l}\text { Downwash Velocities, mph, at Indicated Gross Aircraft Weights, lb, } \\
\text { Disc Loads, psf, and Headings, deg }\end{array}$} \\
\hline & & $\overline{0}$ & 30 & 60 & 90 & $\frac{3}{120}$ & $\frac{3007}{15}$ & $\frac{9.78}{180}$ & & 10 & 240 & 2 & & $\sqrt{320}$ \\
\hline & & deg & deg & deg & deg & deg & de & deg & & $\begin{array}{l}10 \\
\text { eg }\end{array}$ & $\begin{array}{l}240 \\
\text { deg }\end{array}$ & $\begin{array}{l}270 \\
\text { deg }\end{array}$ & $\begin{array}{l}300 \\
\text { deg }\end{array}$ & $\begin{array}{l}\text { deg } \\
\end{array}$ \\
\hline 20 & 0.3 & 32 & 48 & 50 & 54 & 40 & 5 & 58 & & 50 & 51 & 28 & 36 & 48 \\
\hline 30 & $\begin{array}{l}0.3 \\
1.0 \\
2.0 \\
3.0 \\
4.0 \\
5.0 \\
6.0\end{array}$ & $\begin{array}{l}55 \\
46 \\
58 \\
57 \\
46 \\
52 \\
52\end{array}$ & $\begin{array}{l}61 \\
64 \\
64 \\
66 \\
50 \\
46 \\
38\end{array}$ & $\begin{array}{l}62 \\
71 \\
71 \\
64 \\
50 \\
45 \\
28\end{array}$ & $\begin{array}{l}65 \\
66 \\
66 \\
63 \\
44 \\
40 \\
30\end{array}$ & $\begin{array}{l}65 \\
64 \\
67 \\
59 \\
43 \\
39 \\
49\end{array}$ & $\begin{array}{l}5 \\
5 \\
6 \\
6 \\
5 \\
5 \\
5\end{array}$ & $\begin{array}{l}58 \\
76 \\
77 \\
67 \\
58 \\
58 \\
58\end{array}$ & & $\begin{array}{l}65 \\
61 \\
66 \\
63 \\
50 \\
40 \\
43\end{array}$ & $\begin{array}{l}60 \\
62 \\
73 \\
70 \\
56 \\
55 \\
44\end{array}$ & $\begin{array}{l}51 \\
51 \\
63 \\
60 \\
53 \\
55 \\
55\end{array}$ & $\begin{array}{l}53 \\
50 \\
60 \\
59 \\
52 \\
57 \\
58\end{array}$ & $\begin{array}{l}65 \\
65 \\
67 \\
62 \\
48 \\
49 \\
39\end{array}$ \\
\hline 40 & 0.3 & 54 & 61 & 52 & 52 & 53 & 5 & 53 & & 55 & 57 & 57 & 51 & 50 \\
\hline 50 & $\begin{array}{l}0.3 \\
1.0 \\
2.0 \\
3.0 \\
4.0 \\
5.0 \\
6.0\end{array}$ & $\begin{array}{l}80 \\
73 \\
60 \\
58 \\
51 \\
46 \\
34\end{array}$ & $\begin{array}{l}88 \\
74 \\
62 \\
56 \\
43 \\
24 \\
17\end{array}$ & $\begin{array}{l}75 \\
73 \\
62 \\
54 \\
45 \\
36 \\
23\end{array}$ & $\begin{array}{l}79 \\
71 \\
58 \\
51 \\
44 \\
43 \\
25\end{array}$ & $\begin{array}{l}67 \\
70 \\
57 \\
52 \\
44 \\
43 \\
-\end{array}$ & $\begin{array}{l}8 \\
7 \\
6 \\
6 \\
5 \\
5 \\
3\end{array}$ & $\begin{array}{l}78 \\
74 \\
64 \\
60 \\
56 \\
58 \\
41\end{array}$ & & $\begin{array}{l}80 \\
77 \\
64 \\
64 \\
50 \\
42 \\
28\end{array}$ & $\begin{array}{l}76 \\
74 \\
62 \\
58 \\
56 \\
46 \\
32\end{array}$ & $\begin{array}{l}84 \\
77 \\
68 \\
65 \\
50 \\
49 \\
46\end{array}$ & $\begin{array}{l}83 \\
71 \\
58 \\
63 \\
61 \\
64 \\
52\end{array}$ & $\begin{array}{l}79 \\
75 \\
63 \\
58 \\
43 \\
42 \\
24\end{array}$ \\
\hline 60 & 0.3 & 53 & 55 & 51 & 42 & 47 & 4 & 45 & & 50 & 59 & 59 & 61 & 55 \\
\hline 70 & $\begin{array}{l}0.3 \\
1.0 \\
2.0 \\
3.0 \\
4.0 \\
5.0 \\
6.0\end{array}$ & $\begin{array}{l}50 \\
54 \\
40 \\
43 \\
37 \\
32 \\
23\end{array}$ & $\begin{array}{l}52 \\
60 \\
44 \\
49 \\
39 \\
32 \\
26\end{array}$ & $\begin{array}{l}50 \\
56 \\
44 \\
52 \\
44 \\
36 \\
23\end{array}$ & $\begin{array}{l}52 \\
55 \\
43 \\
49 \\
39 \\
38 \\
36\end{array}$ & $\begin{array}{l}48 \\
52 \\
36 \\
41 \\
-- \\
37 \\
32\end{array}$ & $\begin{array}{l}5 \\
5 \\
4 \\
3 \\
3 \\
3 \\
3\end{array}$ & $\begin{array}{l}54 \\
55 \\
45 \\
47 \\
45 \\
45 \\
-\end{array}$ & & $\begin{array}{l}44 \\
47 \\
34 \\
47 \\
42 \\
42 \\
34\end{array}$ & $\begin{array}{l}52 \\
56 \\
46 \\
50 \\
40 \\
44 \\
42\end{array}$ & $\begin{array}{l}61 \\
62 \\
49 \\
56 \\
47 \\
48 \\
44\end{array}$ & $\begin{array}{l}49 \\
47 \\
40 \\
45 \\
40 \\
36 \\
38\end{array}$ & $\begin{array}{l}50 \\
56 \\
44 \\
47 \\
35 \\
35 \\
31\end{array}$ \\
\hline 80 & 0.3 & 37 & 42 & 40 & 39 & 39 & 3 & 39 & & 34 & 42 & 44 & 41 & 36 \\
\hline 90 & $\begin{array}{l}0.3 \\
1.0 \\
2.0 \\
3.0 \\
4.0 \\
5.0 \\
6.0\end{array}$ & $\begin{array}{l}42 \\
50 \\
48 \\
46 \\
51 \\
32 \\
43\end{array}$ & $\begin{array}{l}45 \\
59 \\
51 \\
45 \\
51 \\
33 \\
38\end{array}$ & $\begin{array}{l}47 \\
54 \\
53 \\
50 \\
58 \\
38 \\
48\end{array}$ & $\begin{array}{l}43 \\
54 \\
50 \\
48 \\
57 \\
36 \\
52\end{array}$ & $\begin{array}{l}44 \\
52 \\
48 \\
45 \\
50 \\
37 \\
48\end{array}$ & $\begin{array}{l}3 \\
4 \\
4 \\
4 \\
5 \\
3 \\
4\end{array}$ & $\begin{array}{l}48 \\
58 \\
57 \\
53 \\
58 \\
43 \\
58\end{array}$ & & $\begin{array}{l}38 \\
48 \\
48 \\
46 \\
53 \\
33 \\
38\end{array}$ & $\begin{array}{l}49 \\
62 \\
55 \\
51 \\
55 \\
32 \\
47\end{array}$ & $\begin{array}{l}51 \\
62 \\
60 \\
53 \\
56 \\
42 \\
54\end{array}$ & $\begin{array}{l}42 \\
48 \\
45 \\
48 \\
55 \\
35 \\
45\end{array}$ & $\begin{array}{l}44 \\
57 \\
55 \\
52 \\
51 \\
29 \\
40\end{array}$ \\
\hline 100 & 0.3 & 37 & 40 & 42 & 44 & 41 & 3 & 42 & & 35 & 41 & 46 & 37 & 40 \\
\hline 110 & $\begin{array}{l}0.3 \\
1.0 \\
2.0 \\
3.0 \\
4.0 \\
5.0 \\
6.0\end{array}$ & $\begin{array}{l}34 \\
45 \\
47 \\
42 \\
35 \\
36 \\
28\end{array}$ & $\begin{array}{l}37 \\
46 \\
46 \\
43 \\
27 \\
41 \\
32\end{array}$ & $\begin{array}{l}39 \\
47 \\
44 \\
49 \\
45 \\
47 \\
40\end{array}$ & $\begin{array}{l}39 \\
48 \\
52 \\
49 \\
40 \\
41 \\
30\end{array}$ & $\begin{array}{l}37 \\
49 \\
50 \\
48 \\
41 \\
42 \\
31\end{array}$ & $\begin{array}{l}3 \\
3 \\
4 \\
4 \\
3 \\
3 \\
2\end{array}$ & $\begin{array}{l}32 \\
47 \\
56 \\
54 \\
52 \\
55 \\
47\end{array}$ & & $\begin{array}{l}30 \\
37 \\
42 \\
41 \\
36 \\
40 \\
29\end{array}$ & $\begin{array}{l}39 \\
48 \\
51 \\
48 \\
40 \\
41 \\
31\end{array}$ & $\begin{array}{l}44 \\
55 \\
54 \\
50 \\
44 \\
46 \\
37\end{array}$ & $\begin{array}{l}34 \\
43 \\
45 \\
44 \\
32 \\
36 \\
28\end{array}$ & $\begin{array}{l}37 \\
47 \\
60 \\
53 \\
46 \\
45 \\
32\end{array}$ \\
\hline 120 & 0.3 & 35 & 30 & 36 & 42 & 33 & 2 & 35 & & 23 & 37 & 38 & 30 & 37 \\
\hline 130 & 0.3 & 35 & 31 & 37 & 40 & 35 & 2 & 35 & & 28 & 35 & 39 & 31 & 38 \\
\hline 140 & 0.3 & 29 & 26 & 33 & 32 & 26 & 2 & 29 & & 26 & 30 & 34 & 28 & 32 \\
\hline 150 & $\begin{array}{l}0.3 \\
1.0 \\
2.0 \\
3.0 \\
4.0 \\
5.0 \\
6.0\end{array}$ & $\begin{array}{l}24 \\
29 \\
30 \\
34 \\
30 \\
27 \\
22\end{array}$ & $\begin{array}{l}19 \\
23 \\
32 \\
36 \\
31 \\
28 \\
24\end{array}$ & $\begin{array}{l}25 \\
26 \\
27 \\
34 \\
33 \\
32 \\
25\end{array}$ & $\begin{array}{l}28 \\
31 \\
36 \\
36 \\
36 \\
37 \\
33\end{array}$ & $\begin{array}{l}20 \\
25 \\
25 \\
31 \\
35 \\
35 \\
32\end{array}$ & $\begin{array}{l}2 \\
2 \\
2 \\
3 \\
2 \\
3 \\
2\end{array}$ & $\begin{array}{l}26 \\
31 \\
36 \\
40 \\
38 \\
42 \\
35\end{array}$ & & $\begin{array}{l}22 \\
22 \\
25 \\
30 \\
30 \\
31 \\
25\end{array}$ & $\begin{array}{l}26 \\
30 \\
31 \\
37 \\
37 \\
41 \\
35\end{array}$ & $\begin{array}{l}31 \\
35 \\
36 \\
39 \\
40 \\
40 \\
29\end{array}$ & $\begin{array}{l}28 \\
28 \\
23 \\
26 \\
24 \\
25 \\
21\end{array}$ & $\begin{array}{l}27 \\
28 \\
31 \\
32 \\
34 \\
32 \\
26\end{array}$ \\
\hline 160 & 0.3 & 25 & 24 & 28 & 32 & 23 & 2 & $3^{2}$ & & 21 & 30 & 29 & 26 & 28 \\
\hline 170 & 0.3 & 18 & 16 & 20 & 24 & 15 & 1 & 28 & & 14 & 25 & 22 & 20 & 18 \\
\hline 180 & 0.3 & 28 & 26 & 31 & 33 & 24 & 2 & 37 & & 21 & 37 & 29 & 24 & 29 \\
\hline 190 & 0.3 & 12 & 15 & 20 & 21 & 13 & 1 & 24 & & 10 & 22 & 16 & 12 & 20 \\
\hline
\end{tabular}


Table 17

Downwash Velocities, $\mathrm{CH}-54$ Hover Tests $40 \mathrm{ft}$ Above Ground

Helicopter Gross Weight, 28,600, 38,000, and 47,000 Ib; Disc Ioad, 7.02,

9.33, and 11.55 psf; Rotor Height Above Ground, $40 \mathrm{ft}$

\begin{tabular}{|c|c|c|c|c|c|c|c|c|c|c|c|c|c|c|}
\hline \multirow[b]{2}{*}{$\begin{array}{c}\text { Horizontal } \\
\text { Distance fram } \\
\text { Rotor Hub }(s) \text {, ft } \\
\end{array}$} & \multirow[b]{2}{*}{$\begin{array}{l}\text { Velocity Point } \\
\text { Height Above } \\
\text { Ground, ft }\end{array}$} & \multicolumn{13}{|c|}{$\begin{array}{c}\text { Downwash Velocities, mph, at Indicated Gross Aircraft Weights, Ib, } \\
\text { Disc Loads, psf, and Headings, deg } \\
28,600 \text { ib: }\end{array}$} \\
\hline & & $\begin{array}{c}0 \\
\text { deg }\end{array}$ & $\begin{array}{l}30 \\
\text { deg }\end{array}$ & $\begin{array}{l}60 \\
\text { deg }\end{array}$ & $\begin{array}{l}90 \\
\text { deg }\end{array}$ & $\begin{array}{r}2 \\
120 \\
\text { deg } \\
\end{array}$ & $\begin{array}{l}\frac{000}{15} \\
\text { de }\end{array}$ & & $\begin{array}{l}7.02 \\
180 \\
\text { deg }\end{array}$ & $\begin{array}{l}\frac{18}{210} \\
\text { deg }\end{array}$ & $\begin{array}{l}240 \\
\text { deg }\end{array}$ & $\begin{array}{l}270 \\
\text { deg }\end{array}$ & $\begin{array}{l}300 \\
\text { deg }\end{array}$ & $\begin{array}{l}330 \\
\text { deg }\end{array}$ \\
\hline 20 & 0.3 & -- & 36 & 39 & 46 & 43 & & & 41 & 43 & 35 & 23 & 25 & 47 \\
\hline 30 & $\begin{array}{l}0.3 \\
1.0 \\
2.0 \\
3.0 \\
4.0 \\
5.0 \\
6.0\end{array}$ & $\begin{array}{l}30 \\
29 \\
36 \\
29 \\
21 \\
27 \\
25\end{array}$ & $\begin{array}{l}43 \\
40 \\
43 \\
40 \\
27 \\
29 \\
35\end{array}$ & $\begin{array}{l}48 \\
48 \\
57 \\
51 \\
38 \\
36 \\
41\end{array}$ & $\begin{array}{l}49 \\
50 \\
56 \\
49 \\
36 \\
37 \\
27\end{array}$ & $\begin{array}{l}46 \\
49 \\
52 \\
47 \\
36 \\
37 \\
33\end{array}$ & & & $\begin{array}{l}46 \\
48 \\
57 \\
53 \\
40 \\
43 \\
42\end{array}$ & $\begin{array}{l}52 \\
53 \\
55 \\
48 \\
41 \\
46 \\
44\end{array}$ & $\begin{array}{l}50 \\
48 \\
57 \\
55 \\
43 \\
46 \\
40\end{array}$ & $\begin{array}{l}43 \\
43 \\
50 \\
47 \\
38 \\
43 \\
43\end{array}$ & $\begin{array}{l}43 \\
36 \\
48 \\
45 \\
36 \\
40 \\
40\end{array}$ & $\begin{array}{l}52 \\
50 \\
54 \\
49 \\
36 \\
36 \\
30\end{array}$ \\
\hline 40 & 0.3 & 42 & 40 & 42 & 42 & 42 & & & 48 & 47 & 53 & 44 & 40 & 42 \\
\hline 50 & $\begin{array}{l}0.3 \\
1.0 \\
2.0 \\
3.0 \\
4.0 \\
5.0 \\
6.0\end{array}$ & $\begin{array}{l}67 \\
54 \\
45 \\
46 \\
46 \\
39 \\
32\end{array}$ & $\begin{array}{l}57 \\
51 \\
38 \\
36 \\
32 \\
30 \\
24\end{array}$ & $\begin{array}{l}57 \\
62 \\
50 \\
47 \\
26 \\
23 \\
21\end{array}$ & $\begin{array}{l}64 \\
55 \\
49 \\
49 \\
38 \\
35 \\
27\end{array}$ & $\begin{array}{l}58 \\
57 \\
49 \\
41 \\
31 \\
28 \\
19\end{array}$ & & & $\begin{array}{l}69 \\
60 \\
51 \\
48 \\
36 \\
28 \\
21\end{array}$ & $\begin{array}{l}72 \\
60 \\
49 \\
45 \\
38 \\
27 \\
22\end{array}$ & $\begin{array}{l}72 \\
67 \\
58 \\
54 \\
45 \\
36 \\
21\end{array}$ & $\begin{array}{l}67 \\
60 \\
49 \\
48 \\
44 \\
40 \\
21\end{array}$ & $\begin{array}{l}73 \\
62 \\
53 \\
47 \\
42 \\
43 \\
37\end{array}$ & $\begin{array}{l}63 \\
55 \\
51 \\
44 \\
36 \\
30 \\
22\end{array}$ \\
\hline 60 & 0.3 & 45 & 32 & 41 & 46 & 34 & & & 43 & 49 & 57 & 43 & 45 & 42 \\
\hline 70 & $\begin{array}{l}0.3 \\
1.0 \\
2.0 \\
3.0 \\
4.0 \\
5.0 \\
6.0\end{array}$ & $\begin{array}{l}40 \\
46 \\
32 \\
33 \\
26 \\
22 \\
16\end{array}$ & $\begin{array}{l}30 \\
27 \\
19 \\
23 \\
22 \\
25 \\
23\end{array}$ & $\begin{array}{l}42 \\
45 \\
31 \\
35 \\
30 \\
28 \\
23\end{array}$ & $\begin{array}{l}34 \\
46 \\
32 \\
30 \\
26 \\
25 \\
19\end{array}$ & $\begin{array}{l}34 \\
35 \\
26 \\
27 \\
22 \\
20 \\
16\end{array}$ & & & $\begin{array}{l}39 \\
41 \\
29 \\
29 \\
31 \\
18 \\
19\end{array}$ & $\begin{array}{l}43 \\
44 \\
26 \\
30 \\
28 \\
27 \\
22\end{array}$ & $\begin{array}{l}44 \\
58 \\
46 \\
44 \\
39 \\
33 \\
23\end{array}$ & $\begin{array}{l}41 \\
47 \\
35 \\
42 \\
33 \\
29 \\
22\end{array}$ & $\begin{array}{l}42 \\
46 \\
32 \\
39 \\
37 \\
41 \\
38\end{array}$ & $\begin{array}{l}38 \\
44 \\
32 \\
36 \\
28 \\
27 \\
21\end{array}$ \\
\hline 80 & 0.3 & 35 & 18 & 34 & 35 & 26 & & & 25 & 32 & 34 & 34 & 32 & 31 \\
\hline 90 & $\begin{array}{l}0.3 \\
1.0 \\
2.0 \\
3.0 \\
4.0 \\
5.0 \\
6.0\end{array}$ & $\begin{array}{l}35 \\
45 \\
43 \\
37 \\
37 \\
19 \\
30\end{array}$ & $\begin{array}{l}24 \\
29 \\
25 \\
25 \\
36 \\
20 \\
30\end{array}$ & $\begin{array}{l}38 \\
50 \\
43 \\
37 \\
43 \\
22 \\
30\end{array}$ & $\begin{array}{l}38 \\
42 \\
40 \\
36 \\
38 \\
14 \\
21\end{array}$ & $\begin{array}{l}30 \\
36 \\
36 \\
38 \\
47 \\
29 \\
42\end{array}$ & & & $\begin{array}{l}29 \\
40 \\
38 \\
35 \\
40 \\
27 \\
38\end{array}$ & $\begin{array}{l}30 \\
42 \\
39 \\
36 \\
39 \\
25 \\
33\end{array}$ & $\begin{array}{l}47 \\
53 \\
48 \\
42 \\
52 \\
33 \\
45\end{array}$ & $\begin{array}{l}37 \\
45 \\
42 \\
37 \\
49 \\
28 \\
36\end{array}$ & $\begin{array}{l}31 \\
41 \\
36 \\
34 \\
38 \\
24 \\
40\end{array}$ & $\begin{array}{l}35 \\
50 \\
43 \\
36 \\
40 \\
26 \\
38\end{array}$ \\
\hline 100 & 0.3 & 33 & 25 & 37 & 36 & 32 & & & 25 & 31 & 35 & 37 & 36 & 33 \\
\hline 110 & $\begin{array}{l}0.3 \\
1.0 \\
2.0 \\
3.0 \\
4.0 \\
5.0 \\
6.0\end{array}$ & $\begin{array}{l}26 \\
31 \\
39 \\
38 \\
28 \\
29 \\
18\end{array}$ & $\begin{array}{l}19 \\
21 \\
27 \\
26 \\
17 \\
24 \\
17\end{array}$ & $\begin{array}{l}30 \\
40 \\
40 \\
37 \\
24 \\
27 \\
16\end{array}$ & $\begin{array}{l}29 \\
32 \\
36 \\
34 \\
26 \\
25 \\
15\end{array}$ & $\begin{array}{l}21 \\
29 \\
36 \\
37 \\
30 \\
34 \\
31\end{array}$ & & & $\begin{array}{l}27 \\
36 \\
37 \\
37 \\
29 \\
31 \\
23\end{array}$ & $\begin{array}{l}24 \\
29 \\
35 \\
36 \\
26 \\
30 \\
18\end{array}$ & $\begin{array}{l}36 \\
41 \\
44 \\
41 \\
37 \\
38 \\
33\end{array}$ & $\begin{array}{l}33 \\
41 \\
44 \\
42 \\
34 \\
37 \\
30\end{array}$ & $\begin{array}{l}25 \\
32 \\
34 \\
34 \\
25 \\
32 \\
26\end{array}$ & $\begin{array}{l}28 \\
38 \\
39 \\
39 \\
32 \\
36 \\
31\end{array}$ \\
\hline 120 & 0.3 & 20 & 7 & 26 & 19 & 17 & & & 23 & 15 & 29 & 26 & 22 & 22 \\
\hline 130 & 0.3 & 24 & 18 & 30 & 24 & 24 & & & 23 & 21 & 26 & 26 & 26 & 26 \\
\hline 140 & 0.3 & 18 & 111 & 27 & 22 & 13 & & & 19 & 14 & 22 & 21 & 19 & 25 \\
\hline 150 & $\begin{array}{l}0.3 \\
1.0 \\
2.0 \\
3.0 \\
4.0 \\
5.0 \\
6.0\end{array}$ & $\begin{array}{l}12 \\
14 \\
20 \\
28 \\
28 \\
29 \\
22\end{array}$ & $\begin{array}{r}6 \\
6 \\
7 \\
16 \\
16 \\
18 \\
14\end{array}$ & $\begin{array}{l}24 \\
27 \\
29 \\
32 \\
31 \\
30 \\
26\end{array}$ & $\begin{array}{l}23 \\
20 \\
31 \\
34 \\
24 \\
25 \\
29\end{array}$ & $\begin{array}{r}9 \\
10 \\
10 \\
20 \\
19 \\
21 \\
16\end{array}$ & & & $\begin{array}{l}14 \\
13 \\
19 \\
25 \\
20 \\
22 \\
17\end{array}$ & $\begin{array}{l}13 \\
14 \\
14 \\
21 \\
20 \\
22 \\
16\end{array}$ & $\begin{array}{l}20 \\
21 \\
21 \\
28 \\
28 \\
32 \\
25\end{array}$ & $\begin{array}{l}14 \\
16 \\
19 \\
26 \\
26 \\
28 \\
22\end{array}$ & $\begin{array}{l}17 \\
19 \\
18 \\
25 \\
24 \\
28 \\
24\end{array}$ & $\begin{array}{l}19 \\
22 \\
19 \\
27 \\
29 \\
33 \\
27\end{array}$ \\
\hline 160 & 0.3 & 18 & 12 & 28 & 21 & 14 & & & 18 & 18 & 27 & 18 & 18 & 24 \\
\hline 170 & 0.3 & 14 & 5 & 19 & 14 & 9 & & & 10 & 12 & 17 & 12 & II & 18 \\
\hline 180 & 0.3 & 21 & 14 & 29 & 18 & 12 & & & 18 & 19 & 25 & 17 & 22 & 24 \\
\hline 190 & 0.3 & 8 & 3 & 22 & 12 & 2 & & & 5 & 10 & 12 & 5 & 14 & 12 \\
\hline
\end{tabular}




\begin{tabular}{|c|c|c|c|c|c|c|c|c|c|c|c|c|c|}
\hline \multirow{3}{*}{$\begin{array}{c}\text { Horizontel } \\
\text { Distance fram } \\
\text { Rotor Hub }(\mathrm{s}), \mathrm{ft}\end{array}$} & \multirow{3}{*}{$\begin{array}{l}\text { Velocity Point } \\
\text { Height Above } \\
\text { Ground, ft }\end{array}$} & \multicolumn{12}{|c|}{$\begin{array}{c}\text { Downwash Velocities, mph, at Indicated Gross Aircraft Weights, lb, } \\
\text { Disc Loods, osf, and Headings, deg }\end{array}$} \\
\hline & & $\overline{0}$ & 30 & 60 & 90 & 120 & 15 & $\frac{2.33}{180}$ & $\frac{s f}{210}$ & 240 & 270 & 300 & $\overline{330}$ \\
\hline & & $\underline{\mathrm{deg}}$ & deg & deg & deg & $\underline{\text { deg }}$ & de & $\underline{\operatorname{deg}}$ & deg & deg & deg & deg & deg \\
\hline 20 & 0.3 & 6 & 33 & 52 & 5 & 55 & 4 & 43 & 51 & 47 & 41 & 20 & 15 \\
\hline 30 & $\begin{array}{l}0.3 \\
1.0 \\
2.0 \\
3.0 \\
4.0 \\
5.0 \\
6.0\end{array}$ & $\begin{array}{l}34 \\
33 \\
47 \\
43 \\
33 \\
38 \\
38\end{array}$ & $\begin{array}{l}55 \\
49 \\
60 \\
58 \\
48 \\
48 \\
45\end{array}$ & $\begin{array}{l}63 \\
56 \\
66 \\
62 \\
47 \\
49 \\
41\end{array}$ & $\begin{array}{l}6 \\
6 \\
68 \\
6 \\
4 \\
4 \\
3\end{array}$ & $\begin{array}{l}56 \\
56 \\
62 \\
56 \\
42 \\
41 \\
28\end{array}$ & $\begin{array}{l}5 \\
4 \\
5 \\
5 \\
4 \\
5 \\
5\end{array}$ & $\begin{array}{l}56 \\
56 \\
64 \\
60 \\
47 \\
50 \\
47\end{array}$ & $\begin{array}{l}61 \\
64 \\
68 \\
60 \\
47 \\
43 \\
41\end{array}$ & $\begin{array}{l}61 \\
58 \\
66 \\
65 \\
56 \\
58 \\
54\end{array}$ & $\begin{array}{l}48 \\
48 \\
56 \\
55 \\
47 \\
54 \\
52\end{array}$ & $\begin{array}{l}41 \\
41 \\
43 \\
41 \\
37 \\
48 \\
40\end{array}$ & $\begin{array}{l}48 \\
46 \\
52 \\
53 \\
42 \\
47 \\
45\end{array}$ \\
\hline 40 & 0.3 & 67 & 60 & 52 & $5 l$ & 51 & 5 & 54 & 51 & 58 & 50 & 43 & 52 \\
\hline 50 & $\begin{array}{l}0.3 \\
1.0 \\
2.0 \\
3.0 \\
4.0 \\
5.0 \\
6.0\end{array}$ & $\begin{array}{l}83 \\
67 \\
62 \\
58 \\
49 \\
55 \\
32\end{array}$ & $\begin{array}{l}30 \\
75 \\
62 \\
59 \\
46 \\
42 \\
31\end{array}$ & $\begin{array}{l}79 \\
76 \\
63 \\
59 \\
53 \\
48 \\
30\end{array}$ & $\begin{array}{l}9 \\
7 \\
6 \\
5 \\
4 \\
4 \\
2\end{array}$ & $\begin{array}{l}74 \\
66 \\
52 \\
50 \\
38 \\
29 \\
23\end{array}$ & $\begin{array}{l}8 \\
7 \\
5 \\
5 \\
5 \\
6 \\
5\end{array}$ & $\begin{array}{l}74 \\
71 \\
59 \\
53 \\
45 \\
38 \\
34\end{array}$ & $\begin{array}{l}72 \\
71 \\
60 \\
55 \\
49 \\
46 \\
38\end{array}$ & $\begin{array}{l}86 \\
78 \\
69 \\
63 \\
52 \\
46 \\
41\end{array}$ & $\begin{array}{l}82 \\
68 \\
54 \\
54 \\
50 \\
49 \\
46\end{array}$ & $\begin{array}{l}77 \\
63 \\
56 \\
51 \\
48 \\
48 \\
42\end{array}$ & $\begin{array}{l}79 \\
72 \\
63 \\
61 \\
49 \\
51 \\
39\end{array}$ \\
\hline 60 & 0.3 & 60 & 56 & 56 & 5 & 50 & 5 & 55 & 52 & 61 & 56 & 56 & 62 \\
\hline 70 & $\begin{array}{l}0.3 \\
1.0 \\
2.0 \\
3.0 \\
4.0 \\
5.0 \\
6.0\end{array}$ & $\begin{array}{l}51 \\
61 \\
40 \\
44 \\
29 \\
29 \\
17\end{array}$ & $\begin{array}{l}52 \\
55 \\
43 \\
44 \\
37 \\
34 \\
25\end{array}$ & $\begin{array}{l}58 \\
65 \\
46 \\
51 \\
38 \\
35 \\
30\end{array}$ & $\begin{array}{l}5 \\
5 \\
4 \\
4 \\
4 \\
4 \\
3\end{array}$ & $\begin{array}{l}46 \\
49 \\
39 \\
51 \\
32 \\
30 \\
27\end{array}$ & $\begin{array}{l}5 \\
5 \\
4 \\
5 \\
4 \\
5 \\
5\end{array}$ & $\begin{array}{l}50 \\
57 \\
39 \\
43 \\
39 \\
40 \\
32\end{array}$ & $\begin{array}{l}47 \\
55 \\
36 \\
39 \\
32 \\
34 \\
21\end{array}$ & $\begin{array}{l}59 \\
65 \\
50 \\
59 \\
51 \\
52 \\
43\end{array}$ & $\begin{array}{l}51 \\
60 \\
41 \\
44 \\
41 \\
43 \\
42\end{array}$ & $\begin{array}{l}50 \\
62 \\
43 \\
43 \\
42 \\
45 \\
46\end{array}$ & $\begin{array}{l}51 \\
53 \\
48 \\
54 \\
48 \\
45 \\
36\end{array}$ \\
\hline 80 & 0.3 & 44 & 42 & 40 & 4 & 35 & 4 & 40 & 35 & 51 & 43 & 41 & 43 \\
\hline 90 & $\begin{array}{l}0.3 \\
1.0 \\
2.0 \\
3.0 \\
4.0 \\
5.0 \\
6.0\end{array}$ & $\begin{array}{l}47 \\
62 \\
55 \\
53 \\
53 \\
29 \\
38\end{array}$ & $\begin{array}{l}46 \\
56 \\
50 \\
50 \\
53 \\
35 \\
47\end{array}$ & $\begin{array}{l}49 \\
60 \\
57 \\
55 \\
55 \\
33 \\
43\end{array}$ & $\begin{array}{l}5 \\
6 \\
6 \\
5 \\
6 \\
4 \\
5\end{array}$ & $\begin{array}{l}41 \\
50 \\
49 \\
45 \\
49 \\
32 \\
35\end{array}$ & $\begin{array}{l}4 \\
5 \\
5 \\
5 \\
6 \\
4 \\
5\end{array}$ & $\begin{array}{l}41 \\
47 \\
49 \\
52 \\
58 \\
35 \\
44\end{array}$ & $\begin{array}{l}38 \\
54 \\
49 \\
41 \\
47 \\
28 \\
37\end{array}$ & $\begin{array}{l}58 \\
72 \\
72 \\
64 \\
64 \\
41 \\
52\end{array}$ & $\begin{array}{l}44 \\
57 \\
60 \\
54 \\
57 \\
38 \\
54\end{array}$ & $\begin{array}{l}51 \\
62 \\
55 \\
48 \\
54 \\
43 \\
60\end{array}$ & $\begin{array}{l}47 \\
60 \\
59 \\
53 \\
59 \\
37 \\
46\end{array}$ \\
\hline 100 & 0.3 & 45 & 47 & 44 & 4 & 34 & 4 & 36 & 34 & 49 & 41 & 46 & 49 \\
\hline 210 & $\begin{array}{l}0.3 \\
1.0 \\
2.0 \\
3.0 \\
4.0 \\
5.0 \\
6.0\end{array}$ & $\begin{array}{l}38 \\
44 \\
46 \\
45 \\
37 \\
38 \\
27\end{array}$ & $\begin{array}{l}37 \\
46 \\
58 \\
54 \\
46 \\
46 \\
37\end{array}$ & $\begin{array}{l}40 \\
52 \\
50 \\
45 \\
39 \\
41 \\
34\end{array}$ & $\begin{array}{l}4 \\
5 \\
5 \\
5 \\
4 \\
4 \\
3\end{array}$ & $\begin{array}{l}25 \\
33 \\
43 \\
40 \\
40 \\
40 \\
33\end{array}$ & $\begin{array}{l}4 \\
5 \\
6 \\
6 \\
5 \\
5 \\
4\end{array}$ & $\begin{array}{l}31 \\
37 \\
39 \\
40 \\
35 \\
35 \\
25\end{array}$ & $\begin{array}{l}25 \\
35 \\
40 \\
40 \\
34 \\
37 \\
28\end{array}$ & $\begin{array}{l}43 \\
48 \\
60 \\
54 \\
46 \\
46 \\
38\end{array}$ & $\begin{array}{l}37 \\
44 \\
44 \\
44 \\
40 \\
44 \\
37\end{array}$ & $\begin{array}{l}38 \\
48 \\
52 \\
48 \\
42 \\
44 \\
37\end{array}$ & $\begin{array}{l}45 \\
52 \\
49 \\
45 \\
41 \\
47 \\
40\end{array}$ \\
\hline 120 & 0.3 & 32 & 40 & 41 & 4 & 31 & 4 & 3I & 18 & 45 & 26 & 34 & 38 \\
\hline 130 & 0.3 & 34 & 38 & 38 & 3 & 34 & 4 & 31 & 27 & 39 & 28 & 35 & 36 \\
\hline 140 & 0.3 & 27 & 34 & 36 & 3 & 26 & 4 & 24 & 10 & 24 & 19 & 23 & 25 \\
\hline 150 & $\begin{array}{l}0.3 \\
1.0 \\
2.0 \\
3.0 \\
4.0 \\
5.0 \\
6.0\end{array}$ & $\begin{array}{l}25 \\
27 \\
30 \\
35 \\
34 \\
35 \\
26\end{array}$ & $\begin{array}{l}30 \\
34 \\
39 \\
43 \\
39 \\
36 \\
30\end{array}$ & $\begin{array}{l}32 \\
34 \\
41 \\
42 \\
41 \\
35 \\
32\end{array}$ & $\begin{array}{l}2 \\
3 \\
3 \\
3 \\
3 \\
4 \\
3\end{array}$ & $\begin{array}{l}20 \\
22 \\
25 \\
32 \\
30 \\
25 \\
18\end{array}$ & $\begin{array}{l}3 \\
3 \\
4 \\
4 \\
4 \\
5 \\
4\end{array}$ & $\begin{array}{l}18 \\
20 \\
26 \\
32 \\
32 \\
31 \\
24\end{array}$ & $\begin{array}{l}13 \\
15 \\
23 \\
29 \\
28 \\
26 \\
22\end{array}$ & $\begin{array}{l}30 \\
33 \\
36 \\
36 \\
38 \\
37 \\
35\end{array}$ & $\begin{array}{l}26 \\
30 \\
35 \\
38 \\
37 \\
37 \\
28\end{array}$ & $\begin{array}{l}24 \\
26 \\
28 \\
32 \\
31 \\
31 \\
26\end{array}$ & $\begin{array}{l}30 \\
29 \\
31 \\
33 \\
36 \\
35 \\
29\end{array}$ \\
\hline 160 & 0.3 & 29 & 21 & 32 & 2 & 20 & 3 & 23 & 15 & 36 & 27 & 26 & 29 \\
\hline 170 & 0.3 & 19 & 15 & 20 & 1 & 16 & 2 & 15 & III & 26 & 24 & 18 & 22 \\
\hline 180 & 0.3 & 27 & 24 & 34 & 2 & 26 & 3 & 26 & 14 & 35 & 33 & 27 & 30 \\
\hline 190 & 0.3 & 14 & 12 & 20 & 2 & 12 & 2 & 15 & 8 & 27 & 20 & 14 & 19 \\
\hline
\end{tabular}




\section{Table 17 (Concluded)}

\begin{tabular}{|c|c|c|c|c|c|c|c|c|c|c|c|c|c|}
\hline \multirow{3}{*}{$\begin{array}{c}\text { Horizontal } \\
\text { Distance from } \\
\text { Rotor Hub }(\mathrm{s}) \text {, ft } \\
\end{array}$} & \multirow{3}{*}{$\begin{array}{l}\text { Velocity Point } \\
\text { Height Above } \\
\text { Ground, ft } \\
\end{array}$} & \multicolumn{12}{|c|}{$\begin{array}{r}\text { Downwash Velocities, mph, at Indicated Gross Alrcraft Weights, lb, } \\
\text { Disc Loads, psf, and Headings, deg }\end{array}$} \\
\hline & & 0 & 30 & 60 & 90 & $\frac{47}{120}$ & 150 & $\frac{11.55}{180}$ & $\frac{18 f}{210}$ & 240 & 270 & 300 & $\overline{330}$ \\
\hline & & deg & deg & $\underline{\text { deg }}$ & deg & deg & deg & deg & deg & deg & des & deg & deg \\
\hline 20 & 0.3 & 30 & 46 & 48 & 66 & 57 & 60 & 55 & 68 & 38 & 54 & 41 & 23 \\
\hline 30 & $\begin{array}{l}0.3 \\
1.0 \\
2.0 \\
3.0 \\
4.0 \\
5.0 \\
6.0\end{array}$ & $\begin{array}{l}48 \\
46 \\
51 \\
46 \\
34 \\
39 \\
41\end{array}$ & $\begin{array}{l}62 \\
59 \\
70 \\
60 \\
44 \\
40 \\
45\end{array}$ & $\begin{array}{l}74 \\
62 \\
70 \\
64 \\
51 \\
49 \\
42\end{array}$ & $\begin{array}{l}62 \\
70 \\
74 \\
60 \\
47 \\
39 \\
22\end{array}$ & $\begin{array}{l}68 \\
60 \\
67 \\
58 \\
38 \\
30 \\
22\end{array}$ & $\begin{array}{l}66 \\
59 \\
60 \\
53 \\
38 \\
42 \\
32\end{array}$ & $\begin{array}{l}56 \\
60 \\
64 \\
59 \\
45 \\
49 \\
44\end{array}$ & $\begin{array}{l}68 \\
61 \\
72 \\
66 \\
47 \\
47 \\
42\end{array}$ & $\begin{array}{l}54 \\
55 \\
64 \\
60 \\
53 \\
59 \\
51\end{array}$ & $\begin{array}{l}67 \\
64 \\
72 \\
67 \\
54 \\
59 \\
52\end{array}$ & $\begin{array}{l}62 \\
60 \\
66 \\
64 \\
51 \\
55 \\
42\end{array}$ & $\begin{array}{l}45 \\
49 \\
57 \\
62 \\
51 \\
45 \\
42\end{array}$ \\
\hline 40 & 0.3 & 47 & 54 & 56 & 52 & 51 & 49 & 48 & 51 & 53 & 58 & 58 & 49 \\
\hline 50 & $\begin{array}{l}0.3 \\
1.0 \\
2.0 \\
3.0 \\
4.0 \\
5.0 \\
6.0\end{array}$ & $\begin{array}{l}70 \\
58 \\
52 \\
51 \\
47 \\
45 \\
33\end{array}$ & $\begin{array}{l}80 \\
73 \\
58 \\
52 \\
41 \\
39 \\
28\end{array}$ & $\begin{array}{l}87 \\
79 \\
64 \\
59 \\
41 \\
33 \\
18\end{array}$ & $\begin{array}{l}64 \\
66 \\
58 \\
58 \\
44 \\
34 \\
20\end{array}$ & $\begin{array}{l}62 \\
59 \\
50 \\
51 \\
40 \\
33 \\
23\end{array}$ & $\begin{array}{l}73 \\
67 \\
53 \\
49 \\
42 \\
35 \\
31\end{array}$ & $\begin{array}{l}70 \\
63 \\
54 \\
53 \\
48 \\
45 \\
30\end{array}$ & $\begin{array}{l}72 \\
72 \\
57 \\
53 \\
50 \\
45 \\
31\end{array}$ & $\begin{array}{l}87 \\
76 \\
62 \\
58 \\
50 \\
44 \\
34\end{array}$ & $\begin{array}{l}79 \\
71 \\
59 \\
57 \\
51 \\
53 \\
41\end{array}$ & $\begin{array}{l}84 \\
75 \\
65 \\
60 \\
48 \\
45 \\
34\end{array}$ & $\begin{array}{l}76 \\
60 \\
54 \\
49 \\
47 \\
48 \\
37\end{array}$ \\
\hline 60 & 0.3 & 51 & 48 & 56 & 49 & 40 & 54 & 48 & 58 & 56 & 50 & 57 & 46 \\
\hline 70 & $\begin{array}{l}0.3 \\
1.0 \\
2.0 \\
3.0 \\
4.0 \\
5.0 \\
6.0\end{array}$ & $\begin{array}{l}49 \\
51 \\
37 \\
41 \\
34 \\
37 \\
38\end{array}$ & $\begin{array}{l}42 \\
49 \\
38 \\
37 \\
25 \\
21 \\
25\end{array}$ & $\begin{array}{l}47 \\
49 \\
36 \\
35 \\
29 \\
30 \\
26\end{array}$ & $\begin{array}{l}49 \\
49 \\
43 \\
46 \\
37 \\
33 \\
30\end{array}$ & $\begin{array}{l}43 \\
46 \\
32 \\
30 \\
26 \\
25 \\
20\end{array}$ & $\begin{array}{l}49 \\
53 \\
39 \\
40 \\
34 \\
31 \\
28\end{array}$ & $\begin{array}{l}49 \\
54 \\
39 \\
39 \\
32 \\
35 \\
35\end{array}$ & $\begin{array}{l}53 \\
65 \\
43 \\
48 \\
37 \\
38 \\
35\end{array}$ & $\begin{array}{l}54 \\
56 \\
40 \\
42 \\
35 \\
35 \\
27\end{array}$ & $\begin{array}{l}50 \\
59 \\
42 \\
49 \\
42 \\
49 \\
45\end{array}$ & $\begin{array}{l}51 \\
61 \\
44 \\
53 \\
47 \\
48 \\
40\end{array}$ & $\begin{array}{l}43 \\
49 \\
37 \\
39 \\
33 \\
31 \\
28\end{array}$ \\
\hline 80 & 0.3 & 42 & 41 & 40 & 40 & 36 & 40 & 40 & 42 & 40 & 41 & 41 & 34 \\
\hline 90 & $\begin{array}{l}0.3 \\
1.0 \\
2.0 \\
3.0 \\
4.0 \\
5.0 \\
6.0\end{array}$ & $\begin{array}{l}36 \\
49 \\
47 \\
46 \\
52 \\
38 \\
42\end{array}$ & $\begin{array}{l}49 \\
56 \\
53 \\
49 \\
51 \\
29 \\
39\end{array}$ & $\begin{array}{l}46 \\
54 \\
50 \\
44 \\
43 \\
28 \\
38\end{array}$ & $\begin{array}{l}39 \\
50 \\
49 \\
48 \\
51 \\
33 \\
45\end{array}$ & $\begin{array}{l}40 \\
45 \\
42 \\
44 \\
49 \\
29 \\
40\end{array}$ & $\begin{array}{l}42 \\
52 \\
52 \\
49 \\
50 \\
34 \\
44\end{array}$ & $\begin{array}{l}54 \\
39 \\
39 \\
32 \\
35 \\
31 \\
42\end{array}$ & $\begin{array}{l}42 \\
47 \\
51 \\
44 \\
54 \\
33 \\
43\end{array}$ & $\begin{array}{l}42 \\
48 \\
52 \\
48 \\
52 \\
38 \\
44\end{array}$ & $\begin{array}{l}44 \\
56 \\
53 \\
51 \\
57 \\
39 \\
51\end{array}$ & $\begin{array}{l}52 \\
56 \\
50 \\
48 \\
59 \\
40 \\
57\end{array}$ & $\begin{array}{l}40 \\
47 \\
43 \\
39 \\
46 \\
26 \\
34\end{array}$ \\
\hline 100 & 0.3 & 31 & 43 & 41 & 43 & 38 & 38 & 49 & 48 & 35 & 39 & 47 & 30 \\
\hline 1110 & $\begin{array}{l}0.3 \\
1.0 \\
2.0 \\
3.0 \\
4.0 \\
5.0 \\
6.0\end{array}$ & $\begin{array}{r}5 \\
14 \\
22 \\
25 \\
23 \\
29 \\
26\end{array}$ & $\begin{array}{l}20 \\
32 \\
35 \\
29 \\
19 \\
24 \\
16\end{array}$ & $\begin{array}{l}35 \\
41 \\
41 \\
39 \\
32 \\
35 \\
31\end{array}$ & $\begin{array}{l}29 \\
41 \\
46 \\
46 \\
39 \\
41 \\
37\end{array}$ & $\begin{array}{l}25 \\
38 \\
39 \\
38 \\
29 \\
33 \\
25\end{array}$ & $\begin{array}{l}32 \\
44 \\
48 \\
44 \\
37 \\
38 \\
30\end{array}$ & $\begin{array}{l}47 \\
43 \\
43 \\
48 \\
31 \\
42 \\
40\end{array}$ & $\begin{array}{l}38 \\
49 \\
53 \\
46 \\
41 \\
36 \\
30\end{array}$ & $\begin{array}{r}35 \\
.46 \\
50 \\
50 \\
39 \\
39 \\
30\end{array}$ & $\begin{array}{l}39 \\
49 \\
56 \\
49 \\
41 \\
41 \\
31\end{array}$ & $\begin{array}{l}42 \\
55 \\
51 \\
44 \\
41 \\
45 \\
38\end{array}$ & $\begin{array}{l}32 \\
38 \\
41 \\
41 \\
33 \\
35 \\
25\end{array}$ \\
\hline 120 & 0.3 & 16 & 25 & 33 & 21 & 22 & 20 & 34 & 23 & 41 & 33 & 34 & 28 \\
\hline 130 & 0.3 & -- & 8 & 25 & 23 & -- & 12 & 32 & 24 & 41 & 31 & 40 & 32 \\
\hline 140 & 0.3 & -- & -- & -- & 2 & -- & 8 & 28 & 2 & 29 & 20 & 35 & 23 \\
\hline 150 & $\begin{array}{l}0.3 \\
1.0 \\
2.0 \\
3.0 \\
4.0 \\
5.0 \\
6.0\end{array}$ & $\begin{array}{l}-- \\
-- \\
-- \\
-- \\
-- \\
--\end{array}$ & $\begin{array}{l}-- \\
-- \\
-- \\
-- \\
--\end{array}$ & $\begin{array}{l}\overline{-} \\
\overline{-} \\
11 \\
13 \\
18 \\
15\end{array}$ & $\begin{array}{r}-- \\
-- \\
-- \\
12 \\
11 \\
10 \\
8\end{array}$ & $\begin{array}{l}-- \\
- \\
- \\
- \\
- \\
- \\
--\end{array}$ & $\begin{array}{l}-- \\
-- \\
-- \\
-- \\
--\end{array}$ & $\begin{array}{r}7 \\
19 \\
29 \\
36 \\
42 \\
36 \\
36\end{array}$ & $\begin{array}{c}-- \\
-- \\
6 \\
4 \\
4\end{array}$ & $\begin{array}{l}13 \\
17 \\
21 \\
22 \\
21 \\
24 \\
18\end{array}$ & $\begin{array}{r}9 \\
18 \\
20 \\
22 \\
22 \\
24 \\
21\end{array}$ & $\begin{array}{l}30 \\
37 \\
41 \\
43 \\
41 \\
45 \\
34\end{array}$ & $\begin{array}{l}14 \\
14 \\
14 \\
21 \\
22 \\
24 \\
22\end{array}$ \\
\hline 160 & 0.3 & -- & -- & -- & -- & -- & -- & 4 & $\therefore$ & 14 & 6 & 22 & 18 \\
\hline 170 & 0.3 & -- & -- & -- & -- & -- & -- & -- & - & -- & -- & 2 & 21 \\
\hline
\end{tabular}


Table 18

Downwash Velocities, CH-54 Hover Tests $80 \mathrm{ft}$ Above Ground

Helicopter Gross Weight, 27,400,38,000, and 45,000 1b; Disc Load, 6.73,

9.33, and 11.05 psf; Rotor Height Above Ground, $80 \mathrm{ft}$

\begin{tabular}{|c|c|c|c|c|c|c|c|c|c|c|c|c|c|}
\hline \multirow{3}{*}{$\begin{array}{c}\text { Horlzontal } \\
\text { Distance from } \\
\text { Rotor Hub (s), ft } \\
\end{array}$} & \multirow{3}{*}{$\begin{array}{l}\text { Velocity Point } \\
\text { Height Above } \\
\text { Ground, ft } \\
\end{array}$} & \multicolumn{12}{|c|}{$\begin{array}{c}\text { Downwash Velocities, mph, at Indicated Gross Aircraft We1ghts, 1b, } \\
\text { Disc Loads, psf, and Headings, deg* }\end{array}$} \\
\hline & & $\overline{0}$ & 30 & 60 & - & 120 & 150 & $\frac{6.73}{180}$ & of & 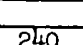 & 270 & 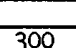 & $\overline{330}$ \\
\hline & & deg & deg & deg & deg & deg & deg & deg & deg & deg & deg & deg & deg \\
\hline 20 & 0.3 & 39 & 44 & 44 & 44 & 41 & 34 & 32 & 41 & 19 & 33 & 20 & 36 \\
\hline 30 & $\begin{array}{l}0.3 \\
1.0 \\
2.0 \\
3.0 \\
4.0 \\
5.0 \\
6.0\end{array}$ & $\begin{array}{l}46 \\
45 \\
48 \\
42 \\
31 \\
29 \\
28\end{array}$ & $\begin{array}{l}39 \\
39 \\
45 \\
37 \\
31 \\
39 \\
26\end{array}$ & $\begin{array}{l}43 \\
45 \\
48 \\
42 \\
30 \\
27 \\
23\end{array}$ & $\begin{array}{l}47 \\
47 \\
52 \\
46 \\
33 \\
35 \\
30\end{array}$ & $\begin{array}{l}43 \\
44 \\
48 \\
43 \\
31 \\
33 \\
28\end{array}$ & $\begin{array}{l}37 \\
39 \\
50 \\
49 \\
38 \\
45 \\
43\end{array}$ & $\begin{array}{l}40 \\
41 \\
48 \\
47 \\
38 \\
43 \\
42\end{array}$ & $\begin{array}{l}45 \\
48 \\
52 \\
51 \\
41 \\
45 \\
45\end{array}$ & $\begin{array}{l}43 \\
40 \\
49 \\
49 \\
38 \\
42 \\
37\end{array}$ & $\begin{array}{l}44 \\
41 \\
48 \\
45 \\
36 \\
42 \\
37\end{array}$ & $\begin{array}{l}35 \\
34 \\
42 \\
43 \\
32 \\
50 \\
36\end{array}$ & $\begin{array}{l}48 \\
46 \\
54 \\
51 \\
38 \\
39 \\
32\end{array}$ \\
\hline 40 & 0.3 & 46 & 33 & 37 & 43 & 41 & 43 & 42 & 43 & 46 & 44 & 39 & 40 \\
\hline 50 & $\begin{array}{l}0.3 \\
1.0 \\
2.0 \\
3.0 \\
4.0 \\
5.0 \\
6.0\end{array}$ & $\begin{array}{l}72 \\
56 \\
45 \\
35 \\
24 \\
18 \\
13\end{array}$ & $\begin{array}{l}49 \\
46 \\
37 \\
37 \\
36 \\
40 \\
34\end{array}$ & $\begin{array}{l}60 \\
50 \\
39 \\
37 \\
30 \\
25 \\
19\end{array}$ & $\begin{array}{l}72 \\
59 \\
51 \\
47 \\
41 \\
34 \\
18\end{array}$ & $\begin{array}{l}62 \\
58 \\
47 \\
46 \\
40 \\
36 \\
27\end{array}$ & $\begin{array}{l}63 \\
57 \\
53 \\
49 \\
41 \\
43 \\
36\end{array}$ & $\begin{array}{l}70 \\
59 \\
45 \\
46 \\
43 \\
44 \\
38\end{array}$ & $\begin{array}{l}65 \\
61 \\
53 \\
49 \\
46 \\
50 \\
40\end{array}$ & $\begin{array}{l}74 \\
69 \\
33 \\
51 \\
43 \\
36 \\
25\end{array}$ & $\begin{array}{l}66 \\
61 \\
50 \\
48 \\
39 \\
35 \\
28\end{array}$ & $\begin{array}{l}60 \\
52 \\
45 \\
48 \\
43 \\
43 \\
31\end{array}$ & $\begin{array}{l}64 \\
64 \\
54 \\
52 \\
42 \\
37 \\
27\end{array}$ \\
\hline 60 & 0.3 & 41 & 33 & 38 & 45 & 42 & 42 & 43 & 45 & 47 & 47 & 39 & 45 \\
\hline 70 & $\begin{array}{l}0.3 \\
1.0 \\
2.0 \\
3.0 \\
4.0 \\
5.0 \\
6.0\end{array}$ & $\begin{array}{l}38 \\
41 \\
28 \\
28 \\
21 \\
21 \\
16\end{array}$ & $\begin{array}{l}32 \\
35 \\
25 \\
26 \\
24 \\
26 \\
27\end{array}$ & $\begin{array}{l}36 \\
43 \\
29 \\
29 \\
25 \\
26 \\
22\end{array}$ & $\begin{array}{l}41 \\
48 \\
36 \\
39 \\
31 \\
24 \\
17\end{array}$ & $\begin{array}{l}43 \\
52 \\
34 \\
37 \\
32 \\
33 \\
30\end{array}$ & $\begin{array}{l}46 \\
51 \\
33 \\
33 \\
28 \\
28 \\
: 27\end{array}$ & $\begin{array}{l}40 \\
45 \\
32 \\
32 \\
25 \\
27 \\
24\end{array}$ & $\begin{array}{l}43 \\
49 \\
35 \\
37 \\
31 \\
37 \\
38\end{array}$ & $\begin{array}{l}47 \\
50 \\
39 \\
39 \\
30 \\
28 \\
27\end{array}$ & $\begin{array}{l}45 \\
49 \\
32 \\
36 \\
31 \\
26 \\
21\end{array}$ & $\begin{array}{l}37 \\
40 \\
30 \\
25 \\
28 \\
30 \\
27\end{array}$ & $\begin{array}{l}41 \\
43 \\
35 \\
41 \\
35 \\
35 \\
32\end{array}$ \\
\hline 80 & 0.3 & 27 & 26 & 28 & 31 & 32 & 41 & 32 & 36 & 36 & 35 & 29 & 22 \\
\hline 90 & $\begin{array}{l}0.3 \\
1.0 \\
2.0 \\
3.0 \\
4.0 \\
5.0 \\
6.0\end{array}$ & $\begin{array}{l}41 \\
48 \\
40 \\
35 \\
37 \\
20 \\
28\end{array}$ & $\begin{array}{l}25 \\
35 \\
34 \\
33 \\
40 \\
29 \\
36\end{array}$ & $\begin{array}{l}29 \\
41 \\
36 \\
31 \\
35 \\
20 \\
31\end{array}$ & $\begin{array}{l}35 \\
42 \\
42 \\
39 \\
39 \\
26 \\
36\end{array}$ & $\begin{array}{l}32 \\
38 \\
41 \\
42 \\
51 \\
29 \\
40\end{array}$ & $\begin{array}{l}32 \\
43 \\
43 \\
44 \\
49 \\
29 \\
34\end{array}$ & $\begin{array}{l}32 \\
42 \\
39 \\
39 \\
44 \\
27 \\
40\end{array}$ & $\begin{array}{l}39 \\
51 \\
48 \\
44 \\
48 \\
30 \\
43\end{array}$ & $\begin{array}{l}34 \\
45 \\
40 \\
37 \\
41 \\
26 \\
38\end{array}$ & $\begin{array}{l}40 \\
45 \\
37 \\
40 \\
45 \\
27 \\
36\end{array}$ & $\begin{array}{l}34 \\
46 \\
40 \\
36 \\
33 \\
23 \\
37\end{array}$ & $\begin{array}{l}30 \\
40 \\
34 \\
32 \\
42 \\
25 \\
34\end{array}$ \\
\hline 100 & 0.3 & 36 & 25 & 33 & 33 & 30 & 31 & 31 & 35 & 28 & 40 & 34 & 31 \\
\hline 110 & $\begin{array}{l}0.3 \\
1.0 \\
2.0 \\
3.0 \\
4.0 \\
5.0 \\
6.0\end{array}$ & $\begin{array}{l}28 \\
38 \\
39 \\
36 \\
27 \\
30 \\
18\end{array}$ & $\begin{array}{l}24 \\
31 \\
35 \\
34 \\
23 \\
28 \\
16\end{array}$ & $\begin{array}{l}27 \\
38 \\
42 \\
34 \\
22 \\
27 \\
18\end{array}$ & $\begin{array}{l}26 \\
36 \\
35 \\
34 \\
25 \\
28 \\
26\end{array}$ & $\begin{array}{l}24 \\
32 \\
35 \\
40 \\
34 \\
36 \\
31\end{array}$ & $\begin{array}{l}29 \\
40 \\
46 \\
47 \\
40 \\
40 \\
28\end{array}$ & $\begin{array}{l}26 \\
31 \\
33 \\
31 \\
22 \\
28 \\
21\end{array}$ & $\begin{array}{l}33 \\
41 \\
39 \\
38 \\
28 \\
32 \\
25\end{array}$ & $\begin{array}{l}23 \\
34 \\
34 \\
29 \\
17 \\
24 \\
20\end{array}$ & $\begin{array}{l}31 \\
40 \\
40 \\
37 \\
27 \\
31 \\
16\end{array}$ & $\begin{array}{l}26 \\
37 \\
41 \\
43 \\
36 \\
37 \\
30\end{array}$ & $\begin{array}{l}27 \\
37 \\
39 \\
34 \\
24 \\
28 \\
19\end{array}$ \\
\hline 120 & 0.3 & 26 & 23 & 25 & 20 & 20 & 29 & 19 & 28 & 19 & 29 & 22 & 20 \\
\hline 130 & 0.3 & 29 & 25 & 26 & 26 & 29. & 28 . & 21. & 31 . & $23^{-}$ & $35^{-}$ & $.26^{-}$ & 28 \\
\hline 140 & 0.3 & 26 & 16 & 21 & 22 & 22 & 20 & 18 & 27 & 19 & 21 & 19 & 22 \\
\hline 150 & $\begin{array}{l}0.3 \\
1.0 \\
2.0 \\
3.0 \\
4.0 \\
5.0 \\
6.0\end{array}$ & $\begin{array}{l}18 \\
22 \\
26 \\
30 \\
27 \\
30 \\
23\end{array}$ & $\begin{array}{r}7 \\
8 \\
8 \\
19 \\
13 \\
17 \\
13\end{array}$ & $\begin{array}{l}17 \\
18 \\
21 \\
27 \\
25 \\
28 \\
22\end{array}$ & $\begin{array}{l}18 \\
19 \\
19 \\
23 \\
19 \\
21 \\
15\end{array}$ & $\begin{array}{l}15 \\
18 \\
19 \\
24 \\
23 \\
24 \\
24\end{array}$ & $\begin{array}{l}15 \\
17 \\
22 \\
31 \\
28 \\
30 \\
22\end{array}$ & $\begin{array}{l}15 \\
14 \\
19 \\
26 \\
24 \\
23 \\
16\end{array}$ & $\begin{array}{l}20 \\
21 \\
27 \\
34 \\
31 \\
35 \\
30\end{array}$ & $\begin{array}{l}15 \\
17 \\
19 \\
28 \\
24 \\
21 \\
17\end{array}$ & $\begin{array}{l}17 \\
17 \\
16 \\
20 \\
18 \\
21 \\
16\end{array}$ & $\begin{array}{l}13 \\
17 \\
15 \\
21 \\
20 \\
21 \\
18\end{array}$ & $\begin{array}{l}17 \\
13 \\
20 \\
26 \\
27 \\
30 \\
24\end{array}$ \\
\hline 160 & 0.3 & 17 & 11 & 23 & 21 & 17 & 20 & 20 & 20 & 18 & 18 & 19 & 21 \\
\hline 170 & 0.3 & 9 & 9 & 15 & 15 & 12 & 13 & 10 & 12 & 12 & 12 & 12 & 14 \\
\hline 180 & 0.3 & 17 & 17 & 24 & 19 & 20 & 20 & 19 & 20 & 21 & 17 & 20 & 23 \\
\hline 190 & 0.3 & 6 & 6 & $\stackrel{12}{100}$ & $\begin{array}{ll}11 \\
\text { tinue }\end{array}$ & 8 & 10 & 8 & 6 & 10 & 8 & 6 & 11 \\
\hline
\end{tabular}




\begin{tabular}{|c|c|c|c|c|c|c|c|c|c|c|c|c|c|}
\hline \multirow[b]{2}{*}{$\begin{array}{l}\text { Horizontal } \\
\text { Distance from } \\
\text { Rotor Hub(s), ft }\end{array}$} & \multirow[b]{2}{*}{$\begin{array}{l}\text { Velocity Point } \\
\text { Height Above } \\
\text { Ground, ft } \\
\end{array}$} & \multicolumn{12}{|c|}{$\begin{array}{c}\text { Downwash Velocities, mph, at Indicated Gross Aircraft Welghts, 1b, } \\
\text { Disc Loads, psf, and Headings, deg } \\
38,000 \text { ib: } 9.33 \text { psf }\end{array}$} \\
\hline & & $\begin{array}{c}0 \\
\text { deg }\end{array}$ & $\begin{array}{c}30 \\
\text { deg }\end{array}$ & $\begin{array}{c}60 \\
\text { deg }\end{array}$ & $\begin{array}{c}90 \\
\text { deg }\end{array}$ & $\begin{array}{l}38, \\
120 \\
\text { deg }\end{array}$ & $\begin{array}{l}001 \mathrm{ib} \\
\mathrm{deg}\end{array}$ & $\begin{array}{l}\frac{9.33 p}{180} \\
\text { deg }\end{array}$ & 210 & $\begin{array}{l}240 \\
\text { deg }\end{array}$ & $\begin{array}{l}270 \\
\text { deg }\end{array}$ & $\begin{array}{l}300 \\
\text { deg }\end{array}$ & $\begin{array}{l}330 \\
\text { deg }\end{array}$ \\
\hline 20 & 0.3 & 15 & 24 & 53 & 56 & 51 & 46 & 37 & 43 & 16 & 40 & 34 & 11 \\
\hline 30 & $\begin{array}{l}0.3 \\
1.0 \\
2.0 \\
3.0 \\
4.0 \\
5.0 \\
6.0\end{array}$ & $\begin{array}{l}37 \\
35 \\
48 \\
41 \\
34 \\
40 \\
42\end{array}$ & $\begin{array}{l}48 \\
44 \\
53 \\
52 \\
44 \\
45 \\
41\end{array}$ & $\begin{array}{l}66 \\
57 \\
66 \\
61 \\
42 \\
41 \\
32\end{array}$ & $\begin{array}{l}59 \\
60 \\
61 \\
58 \\
40 \\
42 \\
34\end{array}$ & $\begin{array}{l}53 \\
53 \\
64 \\
60 \\
46 \\
46 \\
48\end{array}$ & $\begin{array}{l}49 \\
51 \\
62 \\
63 \\
50 \\
53 \\
50\end{array}$ & $\begin{array}{l}45 \\
46 \\
54 \\
55 \\
47 \\
51 \\
50\end{array}$ & $\begin{array}{l}55 \\
59 \\
64 \\
55 \\
47 \\
46 \\
48\end{array}$ & $\begin{array}{l}38 \\
40 \\
48 \\
52 \\
42 \\
47 \\
48\end{array}$ & $\begin{array}{l}49 \\
48 \\
55 \\
56 \\
51 \\
53 \\
50\end{array}$ & $\begin{array}{l}43 \\
41 \\
49 \\
46 \\
41 \\
46 \\
43\end{array}$ & $\begin{array}{l}38 \\
37 \\
45 \\
45 \\
34 \\
40 \\
42\end{array}$ \\
\hline 40 & 0.3 & 46 & 53 & 57 & 50 & 49 & 44 & 48 & 52 & 48 & 47 & 51 & 47 \\
\hline 50 & $\begin{array}{l}0.3 \\
1.0 \\
2.0 \\
3.0 \\
4.0 \\
5.0 \\
6.0\end{array}$ & $\begin{array}{l}83 \\
68 \\
57 \\
50 \\
48 \\
54 \\
42\end{array}$ & $\begin{array}{l}82 \\
72 \\
61 \\
56 \\
50 \\
47 \\
34\end{array}$ & $\begin{array}{l}75 \\
67 \\
61 \\
58 \\
46 \\
39 \\
28\end{array}$ & $\begin{array}{l}76 \\
70 \\
57 \\
57 \\
48 \\
40 \\
29\end{array}$ & $\begin{array}{l}68 \\
68 \\
58 \\
54 \\
45 \\
46 \\
35\end{array}$. & $\begin{array}{l}70 \\
64 \\
58 \\
58 \\
50 \\
50 \\
42\end{array}$ & $\begin{array}{l}78 \\
69 \\
63 \\
59 \\
51 \\
51 \\
42\end{array}$ & $\begin{array}{l}71 \\
71 \\
61 \\
52 \\
42 \\
40 \\
38\end{array}$ & $\begin{array}{l}83 \\
72 \\
61 \\
56 \\
46 \\
48 \\
39\end{array}$ & $\begin{array}{l}81 \\
74 \\
62 \\
62 \\
54 \\
58 \\
48\end{array}$ & $\begin{array}{l}79 \\
67 \\
58 \\
58 \\
53 \\
54 \\
38\end{array}$ & $\begin{array}{l}88 \\
66 \\
60 \\
60 \\
53 \\
52 \\
38\end{array}$ \\
\hline 60 & 0.3 & 59 & 52 & 51 & 51 & 47 & 52 & 53 & 53 & 65 & 63 & 54 & 50 \\
\hline 70 & $\begin{array}{l}0.3 \\
1.0 \\
2.0 \\
3.0 \\
4.0 \\
5.0 \\
6.0\end{array}$ & $\begin{array}{l}55 \\
68 \\
48 \\
52 \\
42 \\
40 \\
36\end{array}$ & $\begin{array}{l}48 \\
60 \\
42 \\
43 \\
38 \\
41 \\
38\end{array}$ & $\begin{array}{l}51 \\
56 \\
44 \\
47 \\
38 \\
32 \\
22\end{array}$ & $\begin{array}{l}50 \\
54 \\
40 \\
43 \\
34 \\
35 \\
32\end{array}$ & $\begin{array}{l}43 \\
51 \\
35 \\
41 \\
34 \\
38 \\
33\end{array}$ & $\begin{array}{l}48 \\
54 \\
42 \\
49 \\
45 \\
46 \\
44\end{array}$ & $\begin{array}{l}44 \\
52 \\
38 \\
45 \\
46 \\
42 \\
36\end{array}$ & $\begin{array}{l}49 \\
52 \\
40 \\
49 \\
45 \\
44 \\
43\end{array}$ & $\begin{array}{l}51 \\
57 \\
43 \\
48 \\
38 \\
38 \\
34\end{array}$ & $\begin{array}{l}57 \\
66 \\
46 \\
53 \\
43 \\
42 \\
34\end{array}$ & $\begin{array}{l}51 \\
54 \\
38 \\
41 \\
37 \\
39 \\
34\end{array}$ & $\begin{array}{l}50 \\
55 \\
45 \\
46 \\
41 \\
34 \\
27\end{array}$ \\
\hline 80 & 0.3 & 50 & 42 & 40 & 40 & 32 & 40 & 41 & 40 & 45 & 51 & 42 & 39 \\
\hline 90 & $\begin{array}{l}0.3 \\
1.0 \\
2.0 \\
3.0 \\
4.0 \\
5.0 \\
6.0\end{array}$ & $\begin{array}{l}55 \\
67 \\
60 \\
51 \\
57 \\
42 \\
48\end{array}$ & $\begin{array}{l}46 \\
54 \\
52 \\
49 \\
50 \\
35 \\
43\end{array}$ & $\begin{array}{l}47 \\
56 \\
53 \\
48 \\
52 \\
30 \\
36\end{array}$ & $\begin{array}{l}44 \\
56 \\
52 \\
49 \\
57 \\
36 \\
51\end{array}$ & $\begin{array}{l}50 \\
58 \\
56 \\
48 \\
53 \\
36 \\
45\end{array}$ & $\begin{array}{l}45 \\
55 \\
55 \\
52 \\
60 \\
43 \\
54\end{array}$ & $\begin{array}{l}47 \\
57 \\
58 \\
51 \\
55 \\
39 \\
49\end{array}$ & $\begin{array}{l}46 \\
52 \\
51 \\
51 \\
58 \\
41 \\
45\end{array}$ & $\begin{array}{l}56 \\
69 \\
59 \\
53 \\
53 \\
32 \\
43\end{array}$ & $\begin{array}{l}54 \\
63 \\
59 \\
52 \\
57 \\
41 \\
55\end{array}$ & $\begin{array}{l}48 \\
55 \\
54 \\
54 \\
56 \\
35 \\
48\end{array}$ & $\begin{array}{l}44 \\
54 \\
53 \\
44 \\
51 \\
36 \\
49\end{array}$ \\
\hline 100 & 0.3 & 47 & 39 & 45 & 46 & 43 & 49 & 44. & 41 & 48 & 53 & 42 & 38 \\
\hline 110 & $\begin{array}{l}0.3 \\
1.0 \\
2.0 \\
3.0 \\
4.0 \\
5.0 \\
6.0\end{array}$ & $\begin{array}{l}44 \\
52 \\
51 \\
46 \\
38 \\
40 \\
38\end{array}$ & $\begin{array}{l}36 \\
40 \\
40 \\
42 \\
37 \\
42 \\
34\end{array}$ & $\begin{array}{l}39 \\
50 \\
51 \\
51 \\
42 \\
43 \\
35\end{array}$ & $\begin{array}{l}41 \\
50 \\
52 \\
49 \\
39 \\
40 \\
31\end{array}$ & $\begin{array}{l}36 \\
45 \\
50 \\
47 \\
40 \\
40 \\
34\end{array}$ & $\begin{array}{l}47 \\
56 \\
57 \\
54 \\
47 \\
48 \\
42\end{array}$ & $\begin{array}{l}42 \\
54 \\
52 \\
50 \\
41 \\
43 \\
36\end{array}$ & $\begin{array}{l}34 \\
44 \\
47 \\
48 \\
39 \\
41 \\
35\end{array}$ & $\begin{array}{l}47 \\
55 \\
60 \\
51 \\
48 \\
43 \\
43\end{array}$ & $\begin{array}{l}46 \\
55 \\
55 \\
51 \\
44 \\
48 \\
42\end{array}$ & $\begin{array}{l}39 \\
51 \\
52 \\
53 \\
47 \\
44 \\
34\end{array}$ & $\begin{array}{l}35 \\
47 \\
47 \\
45 \\
42 \\
42 \\
33\end{array}$ \\
\hline 120 & 0.3 & 39 & 27 & 38 & 38 & 32 & 48 & 39 & 32 & 43 & 38 & 38 & 34 \\
\hline 130 & 0.3 & 39 & 29 & 43 & 40 & 35 & 45 & 36 & 34 & 34 & 42 & 37 & 34 \\
\hline 140 & 0.3 & 25 & 18 & 32 & 31 & 21 & 33 & 25 & 1 & 29 & 31 & 26 & 25 \\
\hline 150 & $\begin{array}{l}0.3 \\
1.0 \\
2.0 \\
3.0 \\
4.0 \\
5.0 \\
6.0\end{array}$ & $\begin{array}{l}25 \\
27 \\
32 \\
36 \\
33 \\
34 \\
26\end{array}$ & $\begin{array}{r}20 \\
24 \\
-27 \\
32 \\
32 \\
34 \\
24\end{array}$ & $\begin{array}{l}37 \\
20 \\
40 \\
37 \\
33 \\
33 \\
27\end{array}$ & $\begin{array}{l}33 \\
37 \\
34 \\
33 \\
28 \\
29 \\
22\end{array}$ & $\begin{array}{l}24 \\
27 \\
32 \\
38 \\
39 \\
42 \\
33\end{array}$ & $\begin{array}{l}32 \\
36 \\
-39 \\
41 \\
38 \\
42 \\
33\end{array}$ & $\begin{array}{l}25 \\
31 \\
36 \\
40 \\
41 \\
44 \\
34\end{array}$ & $\begin{array}{l}24 \\
24 \\
26 \\
22 \\
34 \\
36 \\
31\end{array}$ & $\begin{array}{l}32 \\
36 \\
36 \\
37 \\
36 \\
36 \\
30\end{array}$ & $\begin{array}{l}30 \\
31 \\
36 \\
38 \\
41 \\
43 \\
33\end{array}$ & $\begin{array}{l}26 \\
30 \\
36 \\
39 \\
41 \\
43 \\
37\end{array}$ & $\begin{array}{l}26 \\
30 \\
28 \\
30 \\
29 \\
30 \\
26\end{array}$ \\
\hline 160 & 0.3 & 27 & 23 & 15 & 32 & 34 & 32 & 30 & 25 & 30 & 31 & 30 & 29 \\
\hline 170 & 0.3 & 19 & 16 & 23 & 21 & 21 & 24 & 20 & 18 & 21 & 25 & 25 & 20 \\
\hline 180 & 0.3 & 29 & 26 & 36 & 27 & 28 & 32 & 30 & 27 & 31 & 38 & 28 & 26 \\
\hline 190 & 0.3 & 20 & 16 & 22 & 14 & 18 & 21 & 19 & 18 & 21 & 28 & 18 & 16 \\
\hline
\end{tabular}


Table 18 (Concluded)

\begin{tabular}{|c|c|c|c|c|c|c|c|c|c|c|c|c|c|}
\hline \multirow{3}{*}{$\begin{array}{l}\text { Horizontal } \\
\text { Distance from } \\
\text { Rotor Hub }(\mathrm{s}) \text {, ftt } \\
\end{array}$} & \multirow{3}{*}{$\begin{array}{l}\text { Velocity Point } \\
\text { Height Above } \\
\text { Ground, ft } \\
\end{array}$} & \multirow{2}{*}{\multicolumn{12}{|c|}{$\begin{array}{l}\text { Downwash Velocities, mph, at Indicated Gross Aircraft Weights, lb, } \\
\text { Disc Loads, psf, and Headings, deg } \\
45,000 \mathrm{lb} ; 11.05 \mathrm{psf}\end{array}$}} \\
\hline & & & & & & & & & & & & & \\
\hline & & $\begin{array}{c}0 \\
\text { deg }\end{array}$ & $\begin{array}{c}30 \\
\text { deg }\end{array}$ & $\begin{array}{c}60 \\
\text { deg } \\
\end{array}$ & $\begin{array}{c}90 \\
\text { deg }\end{array}$ & $\begin{array}{l}\text { I20 } \\
\text { deg }\end{array}$ & $\begin{array}{l}150 \\
\text { deg }\end{array}$ & $\begin{array}{l}180 \\
\text { deg }\end{array}$ & $\begin{array}{l}210 \\
\text { deg }\end{array}$ & $\begin{array}{l}240 \\
\text { deg }\end{array}$ & $\begin{array}{l}270 \\
\text { deg } \\
\end{array}$ & $\begin{array}{l}300 \\
\text { deg }\end{array}$ & $\begin{array}{l}330 \\
\text { deg } \\
\end{array}$ \\
\hline 20 & 0.3 & 9 & 26 & 57 & 56 & 58 & 55 & 58 & $\cdots$ & 58 & 64 & 58 & 43 \\
\hline 30 & $\begin{array}{l}0.3 \\
1.0 \\
2.0 \\
3.0 \\
4.0 \\
5.0 \\
6.0\end{array}$ & $\begin{array}{l}37 \\
36 \\
40 \\
36 \\
26 \\
34 \\
31\end{array}$ & $\begin{array}{l}43 \\
38 \\
45 \\
44 \\
36 \\
43 \\
47\end{array}$ & $\begin{array}{l}54 \\
56 \\
59 \\
52 \\
33 \\
25 \\
20\end{array}$ & $\begin{array}{l}54 \\
50 \\
57 \\
51 \\
33 \\
30 \\
21\end{array}$ & $\begin{array}{l}50 \\
54 \\
60 \\
54 \\
37 \\
36 \\
26\end{array}$ & $\begin{array}{l}52 \\
58 \\
54 \\
46 \\
35 \\
34 \\
32\end{array}$ & $\begin{array}{l}55 \\
58 \\
56 \\
60 \\
49 \\
48 \\
43\end{array}$ & $\begin{array}{l}-- \\
-- \\
-- \\
-- \\
-- \\
--\end{array}$ & $\begin{array}{l}51 \\
54 \\
57 \\
46 \\
28 \\
29 \\
16\end{array}$ & $\begin{array}{l}61 \\
65 \\
66 \\
58 \\
37 \\
37 \\
28\end{array}$ & $\begin{array}{l}57 \\
58 \\
53 \\
49 \\
34 \\
34 \\
32\end{array}$ & $\begin{array}{l}49 \\
48 \\
49 \\
51 \\
38 \\
39 \\
36\end{array}$ \\
\hline 40 & 0.3 & 44 & 47 & 46 & 42 & 46 & 49 & 45 & -- & 42 & 51 & 52 & 44 \\
\hline 50 & $\begin{array}{l}0.3 \\
1.0 \\
2.0 \\
3.0 \\
4.0 \\
5.0 \\
6.0\end{array}$ & $\begin{array}{l}66 \\
55 \\
49 \\
52 \\
48 \\
44 \\
36\end{array}$ & $\begin{array}{l}66 \\
60 \\
46 \\
49 \\
47 \\
50 \\
43\end{array}$ & $\begin{array}{l}62 \\
60 \\
48 \\
38 \\
33 \\
29 \\
22\end{array}$ & $\begin{array}{l}62 \\
55 \\
45 \\
45 \\
38 \\
29 \\
21\end{array}$ & $\begin{array}{l}63 \\
56 \\
48 \\
50 . \\
36 \\
31 \\
22\end{array}$ & $\begin{array}{l}62 \\
61 \\
50 \\
46 \\
36 \\
32 \\
27\end{array}$ & $\begin{array}{l}64 \\
63 \\
52 \\
55 \\
46 \\
42 \\
36\end{array}$ & $\begin{array}{l}-- \\
-- \\
-- \\
-- \\
-- \\
--\end{array}$ & $\begin{array}{l}50 \\
54 \\
47 \\
43 \\
36 \\
32 \\
20\end{array}$ & $\begin{array}{l}68 \\
62 \\
51 \\
51 \\
47 \\
44 \\
27\end{array}$ & $\begin{array}{l}74 \\
62 \\
46 \\
39 \\
27 \\
22 \\
15\end{array}$ & $\begin{array}{l}72 \\
60 \\
50 \\
49 \\
34 \\
32 \\
25\end{array}$ \\
\hline 60 & 0.3 & 51 & 50 & 41 & 42 & 41 & 40 & 43 & - & 43 & 50 & 51 & 47 \\
\hline 70 & $\begin{array}{l}0.3 \\
1.0 \\
2.0 \\
3.0 \\
4.0 \\
5.0 \\
6.0\end{array}$ & $\begin{array}{l}48 \\
49 \\
35 \\
37 \\
33 \\
33 \\
27\end{array}$ & $\begin{array}{l}45 \\
53 \\
35 \\
40 \\
37 \\
40 \\
40\end{array}$ & $\begin{array}{l}37 \\
41 \\
30 \\
30 \\
27 \\
29 \\
25\end{array}$ & $\begin{array}{l}42 \\
44 \\
30 \\
30 \\
29 \\
29 \\
23\end{array}$ & $\begin{array}{l}34 \\
41 \\
29 \\
31 \\
27 \\
28 \\
28\end{array}$ & $\begin{array}{l}41 \\
43 \\
34 \\
31 \\
24 \\
20 \\
25\end{array}$ & $\begin{array}{l}40 \\
46 \\
34 \\
39 \\
36 \\
28 \\
28\end{array}$ & $\begin{array}{l}-- \\
-- \\
-- \\
-- \\
--\end{array}$ & $\begin{array}{l}44 \\
46 \\
32 \\
30 \\
26 \\
29 \\
27\end{array}$ & $\begin{array}{l}47 \\
52 \\
36 \\
39 \\
32 \\
33 \\
27\end{array}$ & $\begin{array}{l}30 \\
42 \\
32 \\
33 \\
26 \\
27 \\
23\end{array}$ & $\begin{array}{l}37 \\
36 \\
32 \\
33 \\
25 \\
23 \\
23\end{array}$ \\
\hline 80 & 0.3 & 36 & 36 & 27 & 33 & 29 & 33 & 31 & -- & 33 & 40 & 31 & 27 \\
\hline 90 & $\begin{array}{l}0.3 \\
1.0 \\
2.0 \\
3.0 \\
4.0 \\
5.0 \\
6.0\end{array}$ & $\begin{array}{l}41 \\
52 \\
42 \\
38 \\
41 \\
26 \\
31\end{array}$ & $\begin{array}{l}41 \\
48 \\
41 \\
41 \\
48 \\
34 \\
51\end{array}$ & $\begin{array}{l}18 \\
38 \\
37 \\
38 \\
43 \\
27 \\
37\end{array}$ & $\begin{array}{l}37 \\
45 \\
43 \\
42 \\
49 \\
31 \\
39\end{array}$ & $\begin{array}{l}35 \\
44 \\
43 \\
43 \\
49 \\
32 \\
42\end{array}$ & $\begin{array}{l}33 \\
43 \\
38 \\
38 \\
42 \\
21 \\
30\end{array}$ & $\begin{array}{l}39 \\
45 \\
45 \\
49 \\
57 \\
38 \\
46\end{array}$ & $\begin{array}{l}-- \\
-- \\
-- \\
-- \\
-- \\
--\end{array}$ & $\begin{array}{l}34 \\
40 \\
37 \\
39 \\
45 \\
26 \\
35\end{array}$ & $\begin{array}{l}45 \\
66 \\
50 \\
44 \\
50 \\
33 \\
44\end{array}$ & $\begin{array}{l}33 \\
44 \\
40 \\
40 \\
47 \\
26 \\
37\end{array}$ & $\begin{array}{l}30 \\
39 \\
37 \\
36 \\
43 \\
24 \\
33\end{array}$ \\
\hline 100 & 0.3 & 42 & 41 & 6 & 36 & 35 & 31 & 36 & -- & 28 & 39 & 24 & 30 \\
\hline 110 & $\begin{array}{l}0.3 \\
1.0 \\
2.0 \\
3.0 \\
4.0 \\
5.0 \\
6.0\end{array}$ & $\begin{array}{l}42 \\
52 \\
51 \\
44 \\
35 \\
37 \\
30\end{array}$ & $\begin{array}{l}34 \\
44 \\
46 \\
41 \\
37 \\
37 \\
34\end{array}$ & $\begin{array}{r}-6 \\
11 \\
15 \\
12 \\
20 \\
11\end{array}$ & $\begin{array}{l}26 \\
39 \\
42 \\
40 \\
32 \\
35 \\
28\end{array}$ & $\begin{array}{l}25 \\
39 \\
42 \\
40 \\
35 \\
35 \\
25\end{array}$ & $\begin{array}{l}22 \\
33 \\
35 \\
32 \\
23 \\
24 \\
18\end{array}$ & $\begin{array}{l}22 \\
37 \\
42 \\
45 \\
42 \\
46 \\
42\end{array}$ & $\begin{array}{l}-- \\
-- \\
-- \\
-- \\
-- \\
--\end{array}$ & $\begin{array}{l}18 \\
27 \\
30 \\
27 \\
18 \\
25 \\
15\end{array}$ & $\begin{array}{l}30 \\
38 \\
40 \\
41 \\
34 \\
38 \\
30\end{array}$ & $\begin{array}{l}-- \\
13 \\
15 \\
15 \\
15 \\
25 \\
21\end{array}$ & $\begin{array}{l}26 \\
38 \\
34 \\
33 \\
26 \\
27 \\
18\end{array}$ \\
\hline 120 & 0.3 & 32 & 28 & -- & 22 & 3 & -- & 15 & -- & -- & - & -- & 7 \\
\hline 130 & 0.3 & 26 & 30 & -- & 9 & 6 & -- & $\cdots$ & $\cdots$ & -- & -- & -- & 9 \\
\hline 140 & 0.3 & 12 & 15 & $\cdots$ & $=$ & -- & -- & -- & $\therefore$ & -- & - & -- & $=-$ \\
\hline 150 & $\begin{array}{l}0.3 \\
1.0 \\
2.0 \\
3.0\end{array}$ & $\begin{array}{r}9 \\
14 \\
12 \\
12\end{array}$ & $\begin{array}{c}9 \\
14 \\
13 \\
12\end{array}$ & $\begin{array}{l}-- \\
-- \\
-- \\
--\end{array}$ & $\begin{array}{l}-- \\
-- \\
--\end{array}$ & $\begin{array}{l}-- \\
-- \\
--\end{array}$ & $\begin{array}{l}-- \\
-- \\
--\end{array}$ & $\begin{array}{l}-- \\
-- \\
--\end{array}$ & $\begin{array}{l}-- \\
-- \\
--\end{array}$ & $\begin{array}{l}-- \\
-- \\
--\end{array}$ & $\begin{array}{l}-- \\
-- \\
--\end{array}$ & $\begin{array}{l}-- \\
-- \\
--\end{array}$ & $\begin{array}{l}-- \\
-- \\
--\end{array}$ \\
\hline
\end{tabular}


Table 19

Downwash Velocities, CH-54 Fly-By Tests

Helicopter Gross Weight, 27,400, 38,000, and 45,000 1b; Disc Lood, 6.73, 9.33, and 11.05 psf; Rotor Height Above Ground, $28 \mathrm{ft}$

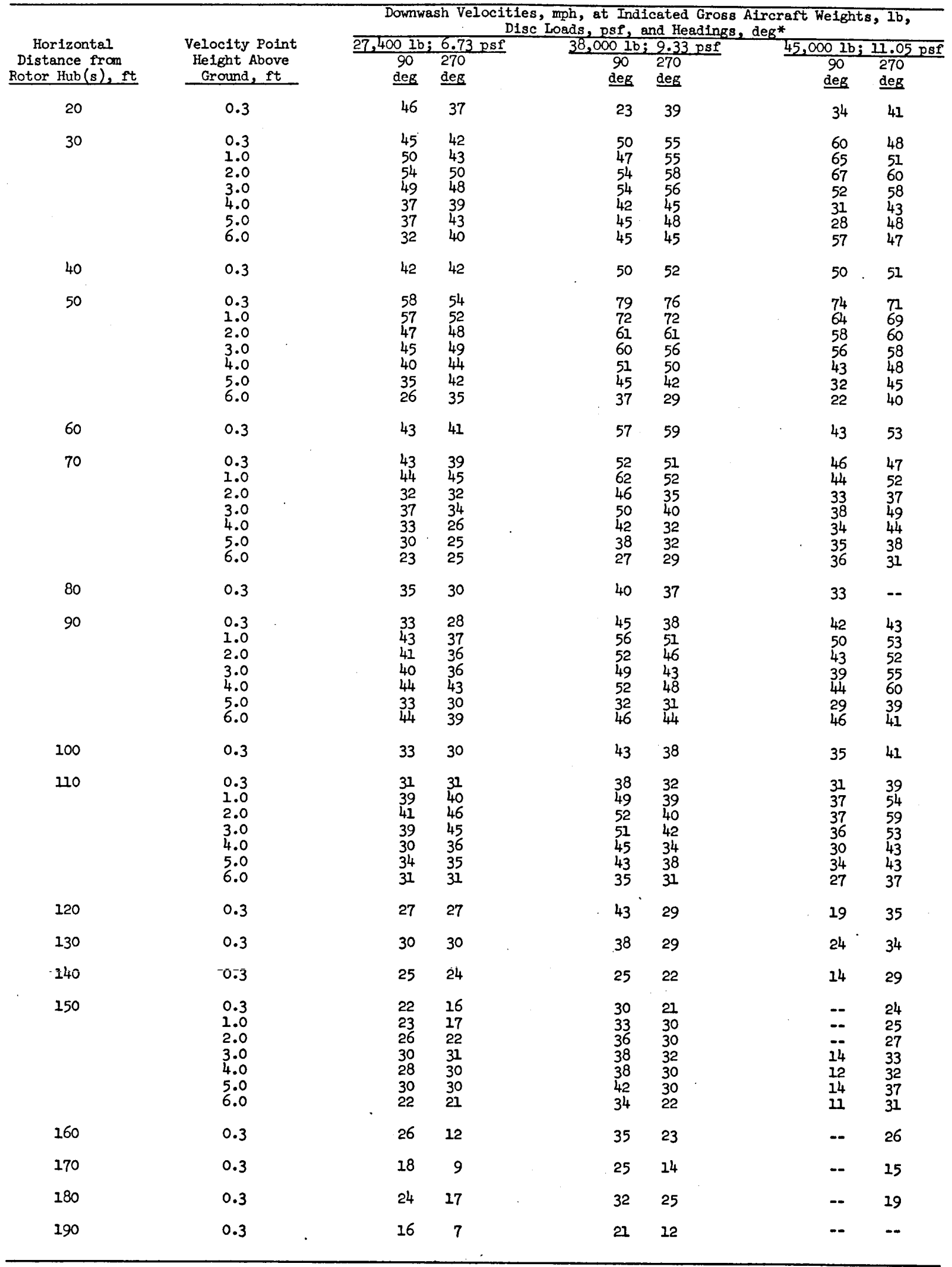


Unclassifled

Security Clessification

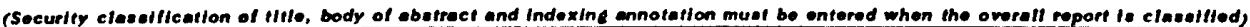
T. ORIGINATING ACTIVITY (Corporalo euthor)

U. S. Army Engineer Waterways Experiment Station Vicksburg, Mississippi

20. RKPORT SECUAITY CLABSIFICATION Unclassified

3. REPORT TITLE

HETICOPTER DOWNWASH DATA

4. DEscalp TIVE NOTEs (Type of repopt end incluelve datea)

Final report

8. AU THOR(s) (Flrat name, middio Inillat, taul namb)

Grady W. Leese

John T. Knight, Jr.

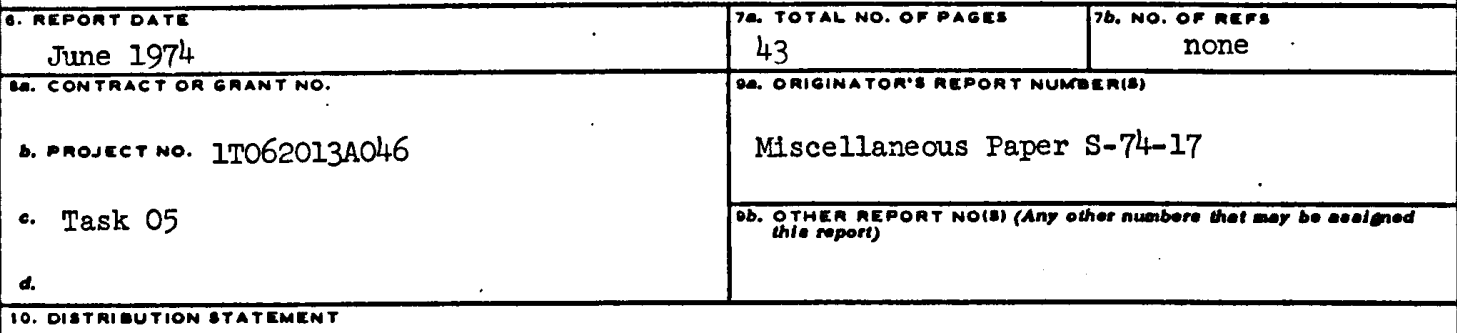

Approved for public release; distribution unlimited.

\begin{tabular}{l|l} 
T1. SUPPLEMENTAAY NOTES & 12. SPONSORING MILITARY ACTIVITY
\end{tabular}

U. S. Army Materiel Command

Washington, D. C.

13. AsstaAcT This data report describes an investigation involving measurement of horizontal velocities, along and up to $6 \mathrm{ft}$ above the ground surface, generated by the rotor systems of various Army helicopters during takeoff, hover, and fly-by operations. Measurements of ground-wash velocitles during varlous operational modes were collected for $0 H-58 A$, OH-6A, AH-IG, UH-IH, UH-IM, CH-47, and CH-54 helicopters. These data are presented for record only with analyses being beyond the scope of this report. 


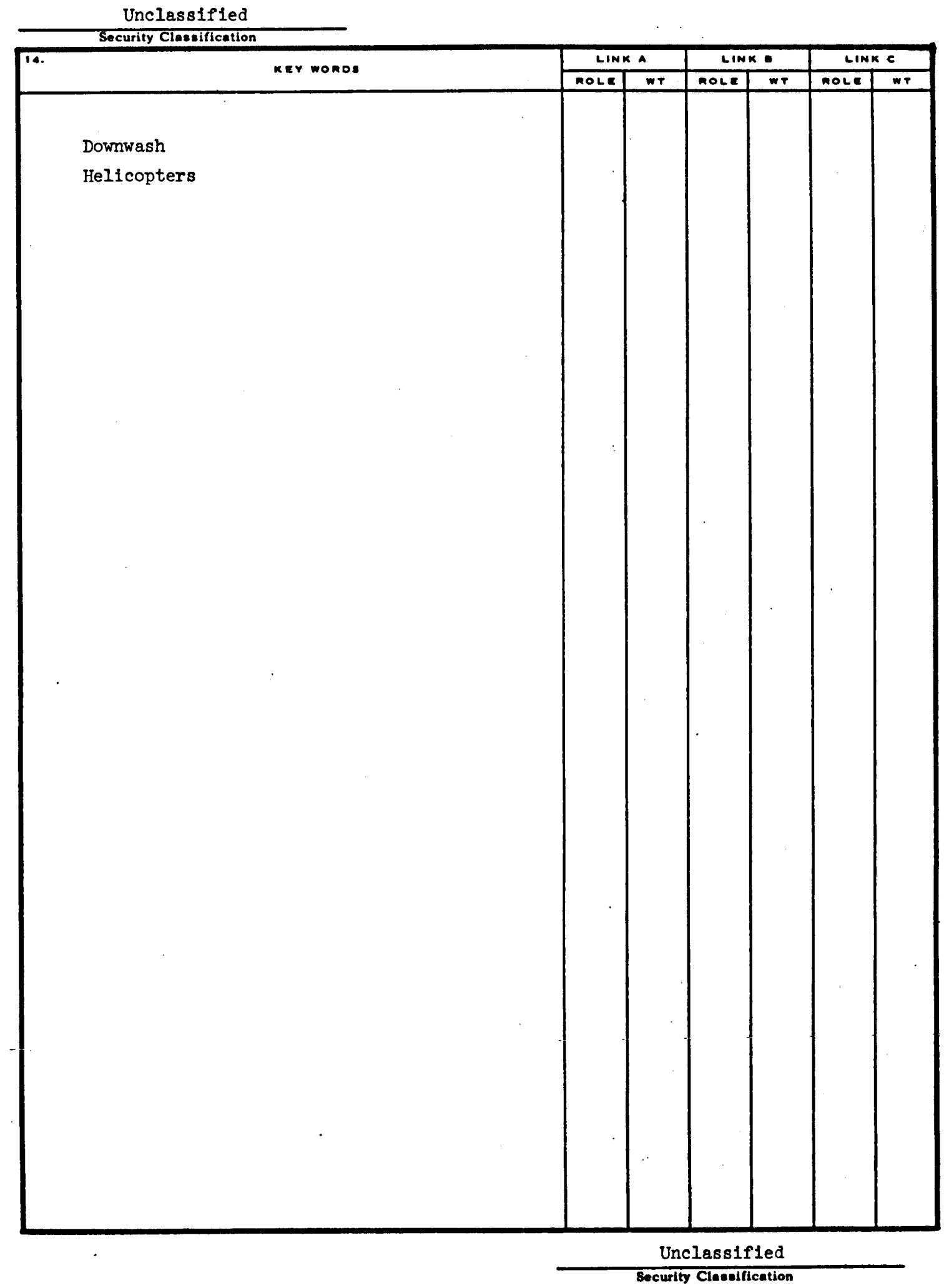

